
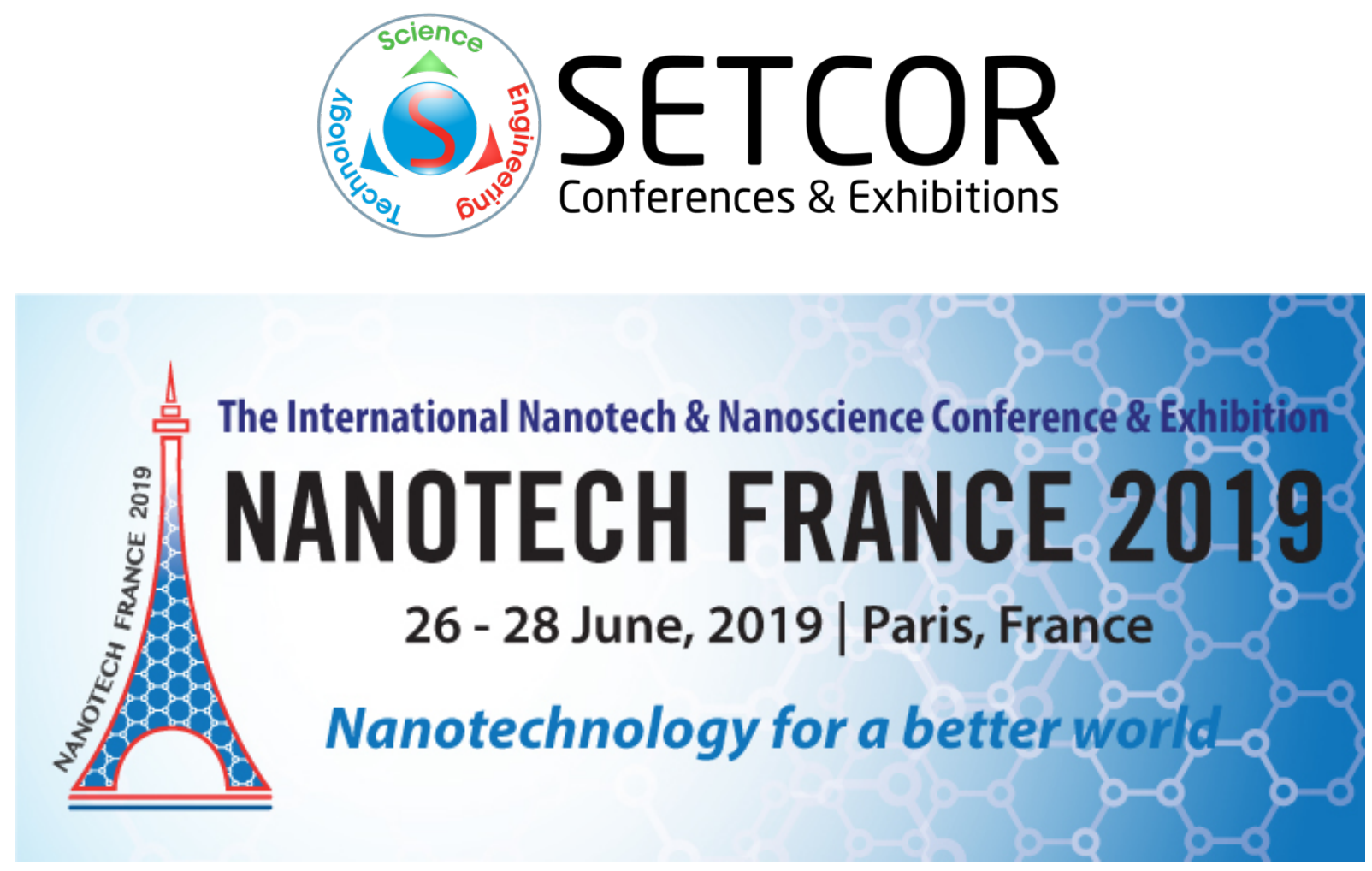

The International Nanotechnology \& Nanoscience Conference \& Exhibition

Nanotech France 2019

Conference Proceedings

DOI: https://doi.org/10.26799/cp-nanotechfrance2019 


\title{
Influence of Plate Acoustic Waves on Electronic Transport in Low Dimensional Nanostructures
}

\author{
Vladimir Kolesov ${ }^{1}$, Iren Kuznetsova ${ }^{1}$, Eugenii Soldatov ${ }^{2}$, Sarkis Dagesyan ${ }^{2}$,Alexander Melnikov ${ }^{2}$ \\ ${ }^{1}$ Kotel'nikov Institute of Radio Engineering and Electronics of RAS \\ Mokhovaya 11, bld.7, Moscow, Russia, kuziren@yandex.ru \\ ${ }^{2}$ Physics Department, M.V. Lomonosov Moscow State University \\ Leninskie gory, 1, bld.2, Moscow, Russia, esold@phys.msu.ru
}

\begin{abstract}
The acoustoelectric effect is the generation of a acoustoelectric current in a non-biased device by a piezoactive acoustic wave. This effect has recently attracted the attention of researchers as possible method to control the motion of massive objects in the quantum regime. We suggest use this effect for development of high sensitive nanoelectronic sensors. The sensitivity could be increase through the use of modulation mode of operation based on the acoustic pilot signal and the transition from quasi-stationary measurement to measurement at the modulation frequency. The acoustic delay line based on shear-horizontal acoustic wave of zero order $\left(\mathrm{SH}_{0}\right)$ with resonant frequency $2.77 \mathrm{MHz}$ in $\mathrm{Y}-\mathrm{X} \mathrm{LiNbO}_{3}$ plate was produced. The nanostructure consisting of nanowire with size $20 \mathrm{~nm}$ x $180 \mathrm{~nm}$ was placed in the center between IDTs. The volt-ampere characteristics of this nanowire in presence and at the absence of the acoustic wave were meazured by picoampermeter. The analysis has shown that presence of piezoactive acoustic wave is influenced on electric current in a nanowire.
\end{abstract}

Keywords: acoustoelectric effect, nanowire, volt-ampere characteristics, shear-horizontal acoustic wave, lithium niobate plate, sensor applications

\section{Introduction}

The acoustoelectric effect is the generation of an acoustoelectric current in a non-biased device by a piezoactive acoustic wave [1]. This effect has recently attracted the attention of researchers as possible method to control the motion of massive objects in the quantum regime [2]. As well now this effect is suggested to apply to production of low power current sources [3], to transfer of charges in semiconductor nanowires [4], etc.

Recently, the works that devoted to the development of hybrid acousto-nano-electronic sensors are appeared. The use of acoustic delay lines with nanostructures located between the interdigital transducers (IDT) is proposed in such sensors [5]. It is assumed that a piezo-active acoustic wave, leading to a displacement of particles of the surface of a medium by a value of the order of tens of angstroms and accompanied by its own electric field, will make an additional contribution to the specific interaction of nano-objects with the environment. In this regard, in this work, the effect of a piezoactive acoustic wave on the current-voltage characteristics of the nanowire located between the IDTs of the delay line is experimentally investigated.

\section{Experimental}

\subsection{Fabrication of the Acoustic Delay Line}

The acoustic delay line and the system of electrodes supplied to the nanostructure were produced as follows. First, the lithium niobate substrate was washed in a centrifuge with a stream of isopropyl alcohol and acetone, and then wiped with dimethylformamide. Then, a Shipley S1813 resist was applied by the centrifuge, after that it was dried for 5 minutes on HotPlate at a temperature of $115^{\circ} \mathrm{C}$. The corresponding photomask was superimposed on the sample in the MA-750 combining unit and the structure was exposed to UV radiation at a wavelength of $400 \mathrm{~nm}$ for 75 seconds at an intensity of $50 \mathrm{~mW} / \mathrm{cm}^{2}$. Next, the sample was placed for 1 minute in a $\mathrm{KOH}$-based developer with a concentration of $0.01 \mathrm{mg} / \mathrm{ml}$. 
After that, thermal evaporation of $100 \mathrm{~nm}$ of titanium and $30 \mathrm{~nm}$ of gold on top of it was sputtered through the obtained photoresist mask. Then, using the lift-off method, the sample was freed from the remnants of the undeveloped photoresist, and the result was a structure of IDT and supply electrodes for the nanostructure. The geometry size of obtained delay line was $11 \mathrm{~mm} \times 13 \mathrm{~mm}$ with substrate thickness $0.35 \mathrm{~mm}$.

\subsection{Fabrication of the Central Nanostructure}

After production of the delay line, the corresponding nanostructure was placed between the IDT. To produce it, we used an electron lithography method with a two-layer overhanging resistive mask. This approach allows to avoid low-quality edges due to the contact of the sprayed metal with the walls of the photoresist and to obtain rather sharp boundaries of metal nanostructures [6]. In result of modified method we obtained central nanostructure with geometry size 80x80 $\mu \mathrm{m}^{2}$.This structure consisted of eight nanowires. Each nanowire was $180 \mathrm{~nm}$ in length, $20 \mathrm{~nm}$ in width and with thickness about $77 \mathrm{~nm}$ (chromium -7nm, gold-50 nm, and titanium- $20 \mathrm{~nm}$ ).

\subsection{Measurement of Volt-Ampere Characteristics of Nanowire}

The block scheme of the measurement setup is shown in figure 1. To excite an acoustic wave with a frequency of 2.77 $\mathrm{MHz}$ in a YX $\mathrm{LiNbO}_{3}$ plate, the input IDT was supplied with voltage from an alternating signal generator G6-34.

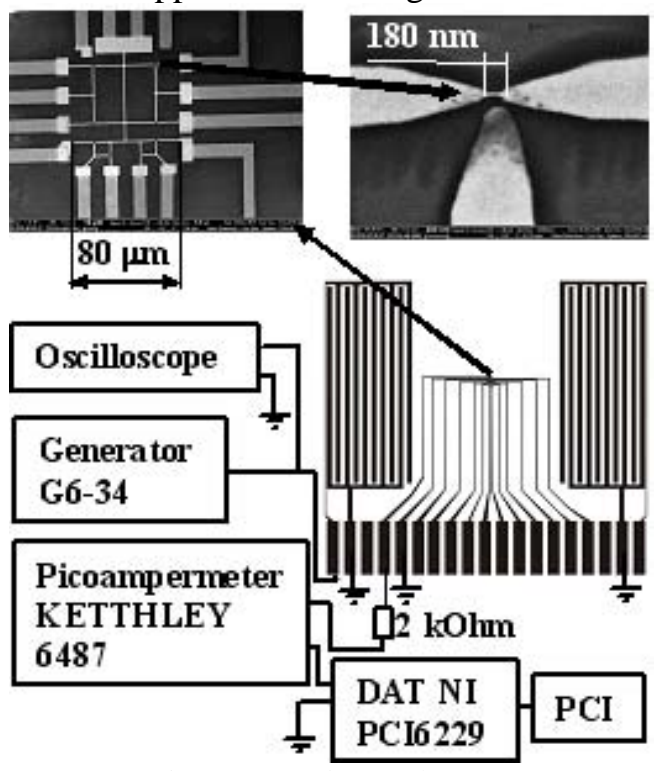

Fig. 1: Experimental setup.

The second IDT was used to receive and monitor the parameters of the transmitted acoustic wave. To measure the currentvoltage characteristics of the nanowire, a picoammeter and a voltage source based on a digital analogous transducer (DAT) were used. The obtained current-voltage characteristics are shown in figure.2.

\section{Results and Discussion}

An analysis of the obtained results has shown that metallic conductivity (Ohm's law) is realized in the nanowire. The black curve in figure 2 corresponds to the voltage-current characteristic of the nanowire in the absence of an acoustic wave in the substrate. The current-voltage characteristic of the nanowire shifts downward at the appearance of an acoustic wave in the substrate due to the appearance of an additional acoustoelectronic electromotive force (EMF). This effect is associated with entrainment of conduction electrons in the nanowire with electric field of the acoustic wave propagating along the nanowire in the piezoelectric substrate.

In the experiment, the dependence of the acoustoelectronic EMF on the amplitude of the excited acoustic wave is observed. The red and green curves in figure 2 correspond to the amplitude of the voltage at IDT $2.5 \mathrm{~V}$ and $7 \mathrm{~V}$, respectively. 


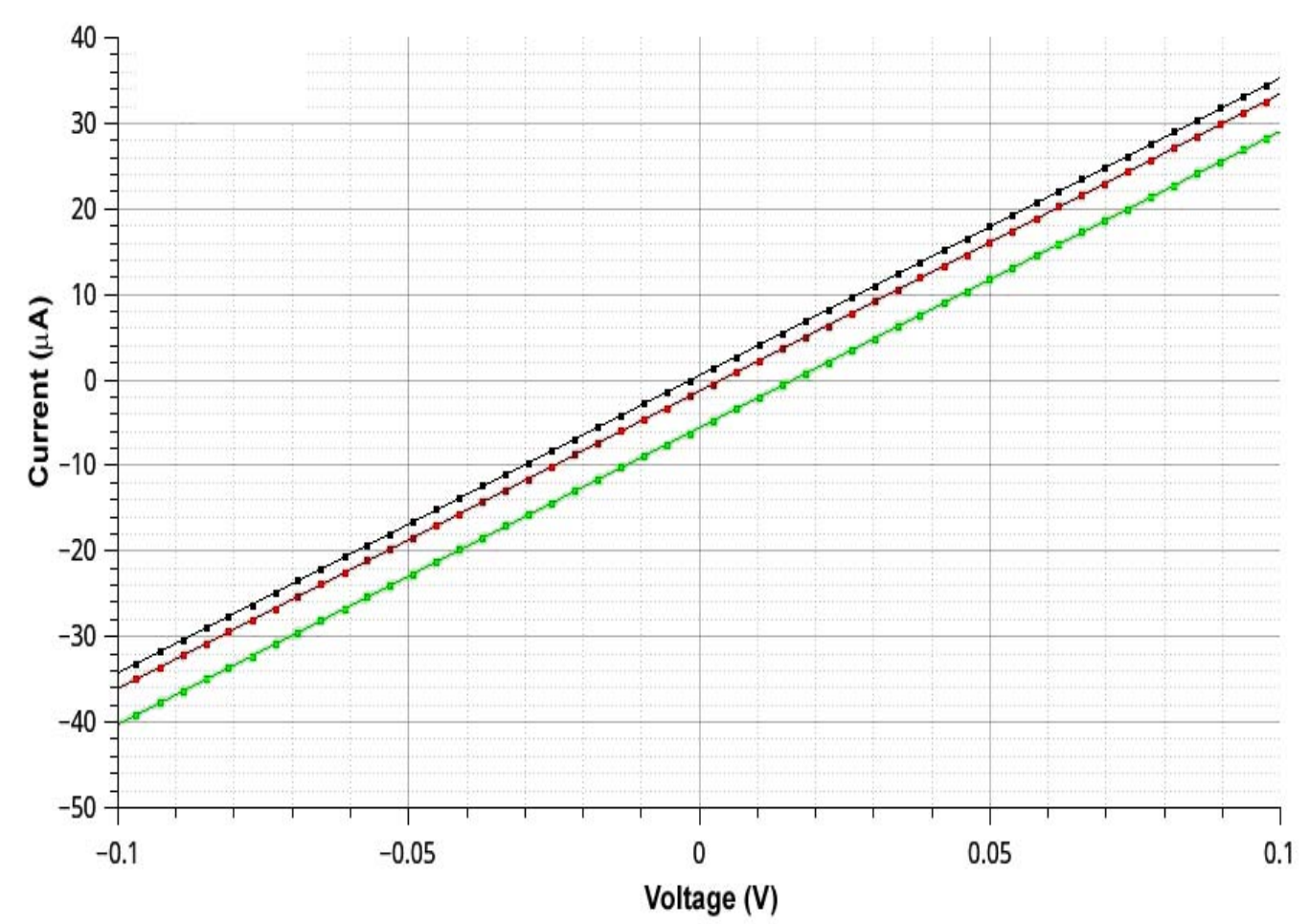

Fig. 1: The current-voltage characteristics of the nanowire at various values of the amplitude of the voltage at input IDT: 0.7V (black), 2.3V (red), 7.5V (green)

\section{Conclusion}

In summary, we modified a method to fabrication of a nanostructure on the surface of piezoelectric plate. In result, we fabricated nanowires with geometrical sizes $180 \mathrm{~nm}$ in length, $20 \mathrm{~nm}$ in width and with thickness $100 \mathrm{~nm}$. The currentvoltage dependencies of this nanowire was measured at various value of the amplitude of the excited acoustic wave. It was found that the current-voltage characteristic of the nanowire shifts downward at the appearance of an acoustic wave in the substrate due to the appearance of an additional acoustoelectronic electromotive force (EMF). This effect is associated with entrainment of conduction electrons in the nanowire with electric field of the acoustic wave propagating along the nanowire in the piezoelectric substrate. This effect could be useful for development of more sensitive hybrid acousto-nanobiosensors.

\section{Acknowledgements}

This work was supported by Russian Science Foundation grant \#18-49-08005 in frame of development of hybrid acoustonanosensor and by Russian Foundation of Basic Research grants \#19-07-01091-a and 18-29-23042-mk in frame of development of technology for production of nanostructures.

\section{References}

1. O. Entin-Wohlman, Y. Levinson and Yu. Galperin, "Acoustoelectric effect in a finite-length ballistic quantum channel,” Phys. Rev. B., vol. 62, no.11, pp. 7283-7288, 2000.

2. Y. Chu, P. Kharel, W. Renninger, L. Burkhart, L. Frunzio, P. Rakich and R. Schoelkopf, “Quantum acoustics with superconducting qubits,” Science, vol. 358, \#6330, pp.199-202, 2017. 
Proceedings of the Nanotech France 2019 International Conference

Paris, France - June 26 - 28, 2019

DOI: https://doi.org/10.26799/cp-nanotechfrance2019

3. X. Dai, F. Zhu, Z.Qian and J.Yang, "Electric potential and carrier distribution in a piezoelectric semiconductor nanowire in time-harmonic bending vibration,"Nano Energy, vol.43, pp. 22-28, 2018

4. S. Roddaro, E. Strambini, L. Romeo, V. Piazza, K. Nilsson, L. Samuelsen and F. Beltram, “Charge pumping in InAs nanowires by surface acoustic waves,” Semicond. Sci. Technol., vol. 25, 024013, 2010.

5. A. Melnikov, E. Soldatov, I. Kuznetsova, V. Kolesov, V. Anisimkin and V. Kashin, “An acousto-electric nanobiosensor,” Bulletin of the Russian Academy of Science Physics, vol.82, \#1, pp.52-54, 2019.

6. Y. Kim, G. Kim, A. Kim, N. Baek, J. Jeong, Y. Han, B.Shin, M. Chung and S. Jung, "Optimisation of bi-layer resist overhang structure formation and $\mathrm{SiO} 2$ sputter-deposition process forfabrication of gold multi-electrode,"RSC Advances, vol.5, \#9, pp.6675-6681, 2015. 


\title{
Effect of UV illumination on Surface Adsorbates in $\mathrm{MoTe}_{2}$ Channel for Gas Sensing Applications
}

\author{
Kyle DiCamillo ${ }^{1}$, Asha Rani ${ }^{2}$, Makarand Paranjape ${ }^{1}$ \\ ${ }^{1}$ Department of Physics, Georgetown University \\ Washington, DC 20057, USA, kd598@georgetown.edu \\ ${ }^{2}$ School of Engineering and Applied Science, The George Washington University \\ Washington, DC 20052, USA
}

\begin{abstract}
The effect of UV illumination on $\mathrm{MoTe}_{2}$ channel is investigated for $\mathrm{NO}_{2}$ and $\mathrm{NH}_{3}$ sensing response. $\mathrm{MoTe}_{2}$ crystals are grown via chemical vapor transport and mechanically exfoliated into few layer flakes for FET fabrication. The $\mathrm{MoTe}_{2}$ FET devices used for this work showed p-type conducting behavior at gate biases between $-60 \mathrm{~V}$ to $60 \mathrm{~V}$. The sensitivity of both $\mathrm{NO}_{2}$ and $\mathrm{NH}_{3}$ gas improves under the influence of UV light. Photoresponse of fabricated MoTe2 devices were tested but found to have unexplained drops in current following UV light exposure. A spike in current is observed after UV exposure followed by gradual decrease until it approaches saturation below the dark current, which is attributed to the release of surface adsorbates (such as oxygen and hydroxyl groups) which are known to dope $\mathrm{MoTe}_{2}$ p-type. Passivation of the surface with a $10 \mathrm{~nm} \mathrm{Al}_{2} \mathrm{O}_{3}$ film prevents changing of surface adsorbents and subsequently was found to eliminate the current drop under UV illumination. It was also found that passivation led to the device switching from p-type to n-type. This is believed to be due to the loss of surface adsorbates during the deposition of the $\mathrm{Al}_{2} \mathrm{O}_{3}$ film. Following the realization that the device behavior is heavily modified by surface adsorbents, variation of carrier gas between $\mathrm{N}_{2}$ and dry breathing air is used during sensing tests. It is found that choice of carrier gas has a large effect on sensing performance for both $\mathrm{NO}_{2}$ and $\mathrm{NH}_{3}$ sensing. $\mathrm{NO}_{2}$ sensing is found to yield better results using $\mathrm{N}_{2}$ carrier gas while $\mathrm{NH}_{3}$ sensing is found to yield better results using dry breathing air as the carrier gas.
\end{abstract}

Keywords: molybdenum ditelluride, two-dimensional materials, nanomaterials, surface

\section{Introduction}

Limitations in scaling of Si-based transistors have drawn attention towards atomically thin two-dimensional (2D) materials such as graphene and transition metal dichalcogenides (TMDs). These semiconducting materials have received significant interest in recent years as new materials for field-effect transistor (FET) applications. Their unique properties have gained them interest for applications in optoelectronics, spintronics and transistor applications. Although graphene has high carrier motilities at room temperature, it is not suitable for logic applications due to absence of band gap. TMDs have a band gap which enable appropriate ON/OFF ratio suitable for current modulation. These are in the form of $\mathrm{MX}_{2}$, where $\mathrm{M}$ is a transition metal and $\mathrm{X}$ is dichalcogenides $(\mathrm{S}$, $\mathrm{Se}$ or Te). The weak van der Waals force between layers enables the mechanical exfoliation of bulk crystals into few or single layer flakes. Because of smaller band gap, single and few layer $\mathrm{MoTe}_{2}$ shows promise for controllable FET and operating range from visible to near-infrared range for optoelectronic devices [1-3]. Since the bandgap of $\mathrm{MoTe}_{2}$ is closer to $\mathrm{Si}(\sim 1.1 \mathrm{eV}), \mathrm{MoTe}_{2}$ is an attractive material for electronic and photovoltaic devices $[1,4]$.

In this work, we have investigated the UV light effect on the surface of $\mathrm{MoTe}_{2}$ flakes. Gradual decay in current after instant spike is observed, as $\mathrm{MoTe}_{2}$ channel is exposed to UV light. This phenomenon of instant increase and gradual decrease of current in presence of UV illumination is attributed to UV-induced carriers and trapping mechanism on the surface of the channel. The UV response is investigated by transient curve various environments.

\section{Results and Discussion}

The $\mathrm{MoTe}_{2}$ crystals used in this work are grown by chemical vapor transport (CVT) method with iodine as transport agent. $\mathrm{MoTe}_{2}$ powder was synthesized by annealing a stoichiometric mixture of molybdenum (99.999\%) and tellurium (99.9\%) powders at $750 \mathrm{C}$ for $72 \mathrm{~h}$ in a sealed quartz ampoule. The $\mathrm{MoTe}_{2}$ powder (approximately $2 \mathrm{~g}$ ) was then mixed with small amount of iodine $(99 / 8 \%, 4 \mathrm{mg} / \mathrm{cm} 3)$ in separate ampoule, sealed and placed in a 
horizontal furnace with temperature gradient so that $\mathrm{MoTe}_{2}$ powder is at $1000{ }^{\circ} \mathrm{C}$ and opposite end is at $950{ }^{\circ} \mathrm{C}$. After 168 hours in the furnace, the ampoule was slow cooled to obtain semiconducting $(2 \mathrm{H}) \mathrm{MoTe}_{2}$ phase crystals. From these bulk crystals few layer flakes were obtained using mechanical exfoliation. These were fabricated into FETs using photolithography. The electrodes consisted of $350 \mathrm{~nm}$ gold on top of $40 \mathrm{~nm}$ titanium. The silicon substrate was used as a back-gate.

Upon exposure to UV light, these $\mathrm{MoTe}_{2}$ a reduction in source/drain current is seen. Figure 4.35 shows a I vs time graph (where the current is normalized to the baseline current and reported as response). In the first third of the graph, the baseline dark current is measured before turning on the ambient room light and noting that the baseline does not shift. Then ultraviolet light is illuminated on the device surface (at $71 \mathrm{~mW} / \mathrm{cm}^{2}$ intensity) and there is an immediate increase in current (which can be attributed to the generation of photocarriers by ultraviolet light) followed by a steady decrease in the current. In the end, the current level stabilizes below the initially-measured dark current baseline of the device. It has been shown previously that MoTe2 will be doped p-type by the presence of oxygen and hydroxyl groups on the surface of the material and the removal of these adsorbates will lead to a shift back towards more n-type behavior [5]. Ultraviolet light has been known to aid in the reduction of surface adsorbates, especially oxygen and hydroxyl groups, in nanomaterials [6]. The reduction in current could be explained by the reduction of surface adsorbates by the ultraviolet light.

As a test of our hypothesis that the reduction of surface adsorbates is causing the reduction in current seen in ptype $\mathrm{MoTe}_{2}$ devices, a passivation layer was added to a p-type $\mathrm{MoTe}_{2}$ that had exhibited the current collapse behavior described above. Figure 4.38 shows the normalized current vs time for this device when exposed to ultraviolet light, and Figure 4.40 shows the transfer and hysteresis characteristics of this device. After these measurements were completed, $10 \mathrm{~nm}$ of passivating $\mathrm{Al}_{2} \mathrm{O}_{3}$ was deposited onto this device via electron-beam (ebeam) evaporation. Figure 4.39 shows the normalized current vs time for this device under ultraviolet exposure under passivation and Figure 4.41 shows the transfer/hysteresis characteristics. Post-passivation, the device switches from p-doped to n-doped. This is likely due to the UHV and elevated temperature during e-beam evaporation thereby reducing surface adsorbates. Now, the device no longer shows the same current drop under ultraviolet exposure since the surface is no longer able to adsorb or desorb oxygen in the same way as before.

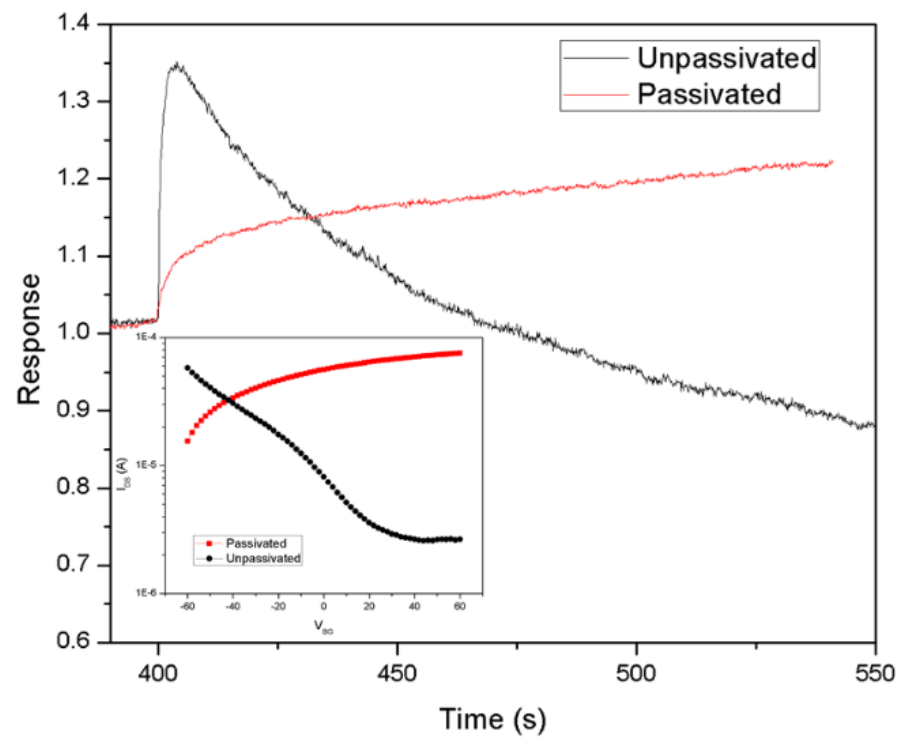

Figure 1: The response vs time for an $\mathrm{MoTe}_{2}$ device before passivation (black curve) and after passivation (red curve). The inset shows the transfer characteristics of the same device before passivation (black curve) and after passivation (red curve). 
The sensing mechanism for semiconducting sensors detecting the presence of $\mathrm{NO}_{2}$ is thought to be due to surface adsorption of $\mathrm{NO}_{2}$ gas molecules onto the semiconductor surface. Since $\mathrm{NO}_{2}$ is a strong acceptor of electrons, it will take free electrons from the semiconducting channel upon adsorption onto the surface. In an n-type device, this should result in a reduction of current upon exposure to $\mathrm{NO}_{2}$ and the reverse should be true of a p-type device. In our sensing data the best results for $\mathrm{NO}_{2}$ detection were obtained using nitrogen carrier gas and ultraviolet illumination. $\mathrm{NH}_{3}$ sensing is also commonly thought to occur through the adsorption of $\mathrm{NH}_{3}$ molecules onto the semiconductor surface. $\mathrm{NH}_{3}$ is a strong electron donor so it should produce the opposite response in both p-type and n-type devices as that for $\mathrm{NO}_{2}$ sensing. However, it has also been shown in metal oxide sensors that $\mathrm{NH}_{3}$ detection can occur through the oxidization of $\mathrm{NH}_{3}$ upon interaction with surface adsorbed oxygen. From our sensing data, using dry air as the carrier gas yields a better result than using nitrogen. This indicates that the additional presence of oxygen actually improved rather than impeded sensing performance in contrast to the
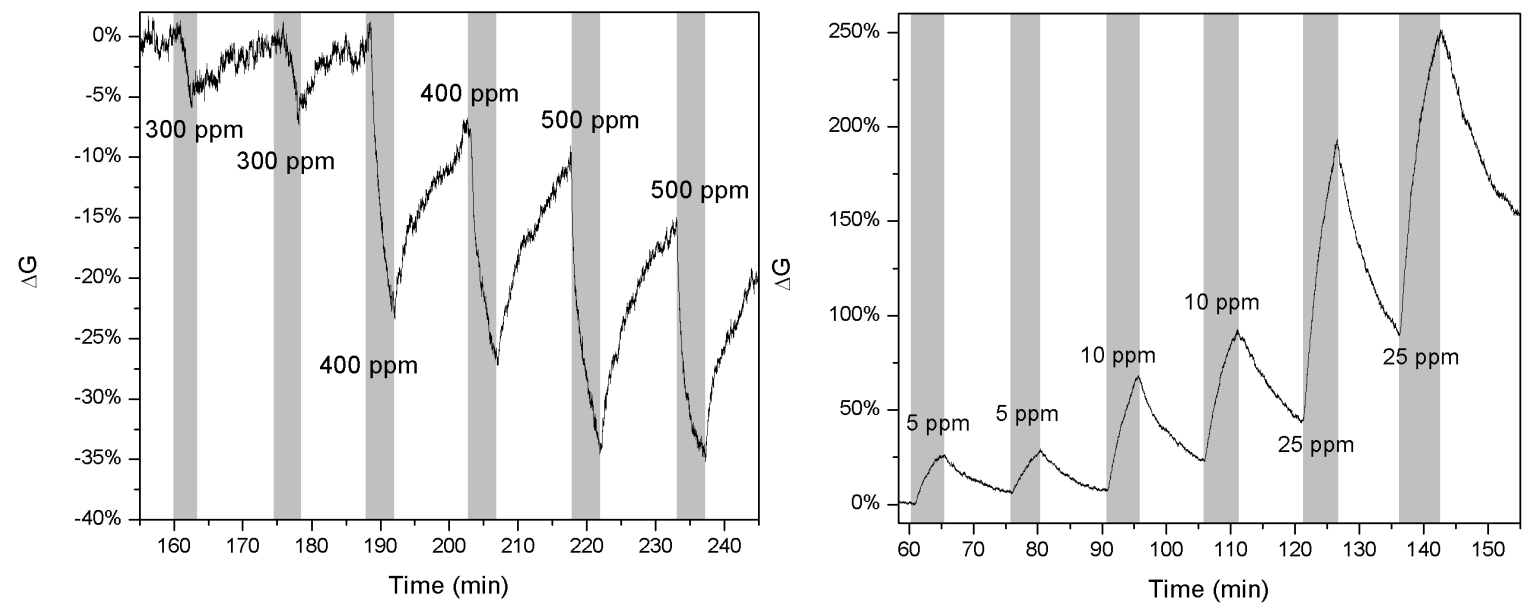

behaviour of our device in response to $\mathrm{NO}_{2}$.

Figure 2: The change in conductance vs time for a $\mathrm{MoTe}_{2}$ device exposed to (left) $\mathrm{NH}_{3}$ gas with dry air carrier gas and (right) $\mathrm{NO}_{2}$ gas with $\mathrm{N}_{2}$ carrier gas. Both measurements were performed with source/drain bias at $5 \mathrm{~V}$ and the $\mathrm{MoTe}_{2}$ device under UV illumination at $71 \mathrm{~mW} / \mathrm{cm}^{2}$ intensity.

\section{Conclusion}

It has been shown here that the sensing behaviour of $\mathrm{MoTe}_{2}$ can be successfully modified through the manipulation of the surface. We have posited here that the differences in behavior shown in our experiments are caused by changes in the amount surface adsorbates present in the $\mathrm{MoTe}_{2}$ sensors. Passivating $\mathrm{MoTe}_{2}$ in order to reduce the surface adsorbates present resulted in a dramatic change in device response to UV illumination. Additionally, passivation caused a change in the polarity of the $\mathrm{MoTe}_{2}$ device, an effect we believe is best explained by a reduction in surface adsorbates. The response of $\mathrm{MoTe}_{2}$ to both $\mathrm{NO}_{2}$ and $\mathrm{NH}_{3}$ gas was measured and we believe that the differences in these responses in different ambient conditions are best explained by changes in surface adsorbates. The $\mathrm{NO}_{2}$ response was best in a $\mathrm{N}_{2}$ ambient environment while the $\mathrm{NH}_{3}$ response became better in a dry air ambient environment. This difference we believe can be explained by differences in the sensing mechanisms of each gas. The $\mathrm{NO}_{2}$ mechanism relies solely on surface adsorption and thus is adversely affected by surface adsorbates. On the other hand, the $\mathrm{NH}_{3}$ mechanism seems to rely on a combination of surface adsorption and chemical interactions between surface oxygen and the $\mathrm{NH}_{3}$ gas which is enhanced by the presence of surface adsorbates.

\section{Acknowledgments}

We would like to acknowledge Albert Davydov and Sergiy Krylyuk of the National Institute for Standards and Technology for providing the $\mathrm{MoTe}_{2}$ bulk crystals that were used to fabricate the $\mathrm{MoTe}_{2}$ devices as described in this work. 


\section{References}

1. S. Mouri, Y. Miyauchi, and K. Matsuda, "Tunable photoluminescence of monolayer MoS2 via chemical doping," Nano Lett., vol. 13, p. 5944, 2013.

2. K. F. Mak, C. Lee, J. Hone, J. Shan, and T. F. Heinz, "Atomically thin MoS2: A new direct gap semiconductor," Phys. Rev. Lett., vol. 105, 2010, Art. no. 136805.

3. T. Wang, R. Zhu, J. Zhuo, Z. Zhu, Y. Shao, and M. Li, "Direct detection of DNA below ppb level based on thionin-functionalized layered MoS2 electrochemical sensors," Anal. Chem., vol. 86, 2014, Art. no. 12064.

4. Y. Ding, Y. Wang, J. Ni, L. Shi, S. Shi, and W. Tang, "First principles study of structural, vibrational and electronic properties of graphene-like MX2 (M = Mo, Nb,W, Ta; X=S, Se, Te) monolayers," Phys. B. vol. 406, p. 2254, 2011

5. Chang, Y., Yang, S., Lin, C., Chen, C., Lien, C., Jian, W., Ueno, K., Suen, Y., Tsukagoshi, K., and Lin, F., "Reversible and Precisely Controllable p/n-Type Doping of MoTe2 Transistors through Electrothermal Doping”, Adv. Mater., 30(13), 1706995(2018).

6. E. Wu, Y. Xie, B. Yuan, H. Zhang, X. Hu, J. Liu, and D. Zhang, "Ultrasensitive and Fully Reversible NO2 Gas Sensing Based on p-Type MoTe2 under Ultraviolet Illumination”, ACS Sens., 3, 1719-1726(2018). 


\title{
Electric Conductivity in Silicone-Carbon Black nanocomposites: percolation and variable range hopping on a fractal
}

\author{
Riadh Neffati $^{1 *}$, J. M. C. Brokken-Zijp ${ }^{2}$ \\ ${ }^{1}$ King Khalid University, Department of Physics, PO. Box 9032 \\ ABHA 61413, Kingdom of Saudi Arabia, riadhneffati@gmail.com or ryad@kku.edu.sa \\ ${ }^{2}$ The Dutch Polymer Institute, Technical University of Eindhoven, PO. Box 513, \\ 5600 MB Eindhoven, The Netherlands
}

\begin{abstract}
Effects on dc conductivity of filler volume fraction, temperature and electric field strength, are discussed using percolation and variable range hopping on a fractal as theoretical background. One also investigates effects of curing rate and CB content on both surface's morphology and conductivity. The fractal aspect of CB network is studied by optical microscopy (OM). Electric conductivity is measured using four points setup. AFM and STM are used to study respectively surface morphology and conductivity. Low percolation threshold $\phi_{\mathrm{C}}=0,15 \%$ and scaling exponent $\mu \sim 2,6$ of conductivity with CB volume fraction. The later exponent is correlated to the measured fractal dimension $\mathrm{d}_{\mathrm{f}}=2,4$. Conduction at low temperature proceeds by mean of variable range hopping on the CB fractal network with a Mott's exponent $\mathrm{m}=0,65$ and temperature $\mathrm{T}_{0}=64 \mathrm{~K}$. Non-Ohmic conductivity curves at different temperatures could be superposed into a unique master curve and the onset field of non-linearity Scales with temperature with a typical exponent $\mathrm{x}_{\mathrm{T}} \sim 1,2$. At low curing rate, capillary forces have time to bring down all $\mathrm{CB}$ particles from the surface into the bulk whereas at fast curing rate the roughness and conductivity of the surface remain high.
\end{abstract}

Keywords: nanocomposites, percolation, fractal, Variable range hopping, Non Ohmic conduction, skin formation

\section{Introduction}

Polymeric nanocomposites are used in many applications such as antistatic material, electromagnetic interference shielding materials [1,2] conductive transparent thin films for touch screens and artificial skins [3], sensors based on change of electric resistance with external parameters $[4,5]$. Fundamental research on conductive polymeric nanocomposite intends to answer different scientific problems concerning the behaviour of electric conductivity when changing the following experimental parameters : (a) The filler volume fraction $\phi$, (b) The temperature $\mathrm{T}$, (c) The electric field strength $\mathrm{F}$ or $(\mathbf{d})$ its frequency $\omega,(\mathbf{e})$ the polymer matrix morphological parameters (molecular weight \& ramification, cross-linking density, crystallinity,...) (f) External parameters (swelling solvent, magnetic field, mechanical stress). One has to explain these major experimental facts with a "unique theoretical picture" supported by morphological and structural investigation. Furthermore, while many applications make use of surface morphology and conductivity which are often different from the balk ones and are processing dependent. The aim of this work is to correlate structural features to the dc conductivity using both percolation and VRH on a fractal, when changing CB volume fraction, temperature, and electric field strength. Then a systematic study by mean of scanning probe microscopy SPM technics show how surface properties (morphology and surface conductivity) are affected by both $\mathrm{CB}$ content and curing rate of the matrix.

\section{Experimental}

Starting from a premix containing $10 \mathrm{wt} .-\%$ of carbon black (Printex XE 2), lower concentrations where prepared by dilution. The used silicone oil for the premix preparation is a vinyl terminated Polydimethylsiloxane (V-PDMS) with typical molecular weight $\mathrm{M}_{\mathrm{n}} \sim 23500$ as estimated by GPC and polydispersity index I 2,5. The density of the polymeric matrix is about $0.9 \mathrm{~g} / \mathrm{cm}^{3}$. A rubbery matrix is obtained by adding a cross-linker (multifunctional siloxane hydride), a catalyst (1ppm Pt) and an inhibitor (1-ethynyl-1-cyclohexanol) for controlling the reaction kinetics. The curing reaction is hydrosililation which is a specific reaction (only end groups react) with no side products. Printex XE 2 is a conductive CB grade from Degussa with a specific surface $950 \mathrm{~m}^{2} / \mathrm{g}$ and a typical particle diameter of about $30-50 \mathrm{~nm}$. These particles are assembled in primary agglomerate of typical size 100-300 $\mathrm{nm}$ [54]. Curing was performed at $120{ }^{\circ} \mathrm{C}$ during 24 hours for bulk samples. For scanning probe microscopy measurements (AFM, STM), different samples droplets were deposited separately on a silicon wafer (Infineon). 
The concentration of $\mathrm{CB}$ is changed and in the other direction the concentration of the inhibitor (and consequently the curing rate) is changing with constant $\mathrm{CB}$ content. The entire wafer with the different samples was held in the same curing condition $\left(120^{\circ} \mathrm{C}\right.$ during 1 hour).

The micrographs of optical microscopy were obtained using an Axioplan microscope with CCD camera. For Atomic Force Microscopy (AFM) or Scanning Tunneling Microscopy (STM) investigations we used both the Solver P7LS and Semina of NT-MDT Ltd. (Moscow - Russia). For bulk DC conductivity measurements at room temperature we used four points setup with a keithley source 237 and keithley electrometer. The low temperature DC conductivity measurements were performed in an oxford instruments CF200 flow cryostat and ICT4 temperature controller cooling rate is about $3 \mathrm{~K} / \mathrm{mn}$. The sample temperature was measured using a $\mathrm{RhFe}$ Thermoeter close to the sample. In order to ovoid sample heating by mean of joule effect, measurements were done by mean of pulse application with pulse's duration about $5 \mathrm{~ms}$. The distance between the 2 gold contact evaporated on the sample is $1,5 \mathrm{~mm}$, the thickness of the sample is about $80 \mu \mathrm{m}$ and its width is $10 \mathrm{~mm}$. One volt drop along the separation distance between gold contacts corresponds to an applied field of $667 \mathrm{~V} / \mathrm{m}$.

\section{Results and discussion}

\subsection{Effects of the filler volume fraction}

Above the percolation thresholds $\phi_{\mathrm{c}}=0,15 \%$ as estimated from dc conductivity measurements, a network of CB spans the sample. The formation of the $\mathrm{CB}$ network is accompanied by a tremendous increase in the dc conductivity. Figure 1a, shows an Optical Microscopy $(\mathrm{OM})$ picture of a cured sample $1 \mathrm{wt}-\%(\phi=0,5 \%)$, revealing the fractal nature of the CB network. Numerical treatment of such picture gives a typical fractal dimension $\mathrm{d}_{\mathrm{f}}$ $=2.404$. The same fractal dimension was deduced from small angle scattering of $\mathrm{x}$ rays on suspensions (before curing) [9]

This Threshold value is well below the theoretical estimation of hard sphere (HS) 16\%. In Printex XE2, high resolution TEM shows that $\mathrm{CB}$ particles are interconnected in primary agglomerates [10] and it is impossible to breakdown theses agglomerates by mixing since many graphitic layers interconnect them. For this reason, one can suppose that we don't have aggregation of CB black particles but of agglomerates (Blobs) with a volume fraction $\phi_{\mathrm{b}}$. The threshold could be written then as : $\phi_{c}=\phi_{H S} \cdot \phi_{b}$. In order to explain the low value of the percolation threshold $\phi_{\mathrm{c}}=0,15 \%$ taking into account the known value of hard sphere percolation threshold $\phi_{H S} \simeq 16 \%$, one has to suppose that the volume fraction of each blob is about $0,94 \%$.

Above the percolation threshold, dc conductivity scales with the $\mathrm{CB}$ volume fraction in a power law way (Figure 1b) as predicted by percolation theory: $\sigma_{D C}(\phi) \cong \sigma_{0} \cdot\left(\phi-\phi_{C}\right)^{\mu}$, with $\mu=2,6$. This measured value is slightly higher than the expected value using the equation $\mu=v \cdot\left(d_{\omega}+d-d_{f}-2\right)$. In fact, the measured fractal dimension $\mathrm{d}_{\mathrm{f}}=$ 2,4 and the correlation length exponent $v=0,8$ in cured samples in this work or suspension [9] when one assumes that Alexander-Orbach [11] relation is valid. In order to find starting from the measured structural exponents $\left(d_{f}, v\right)$ the same exponent $\mu$ as found in dc conductivity measurements, we should suppose that $d_{\omega} / d_{f} \sim 1,8$ rather than 1,5 as stated by Alexander-Orbach (See the corresponding conference paper). The measured scaling exponent of dc conductivity with the filler content is independent of temperature. We also checked this behaviour after and before curing. It seems that curing of the polymeric matrix has no effect also on conduction process. The formation of the polymer network doesn't affect the colloidal network of CB agglomerates. 

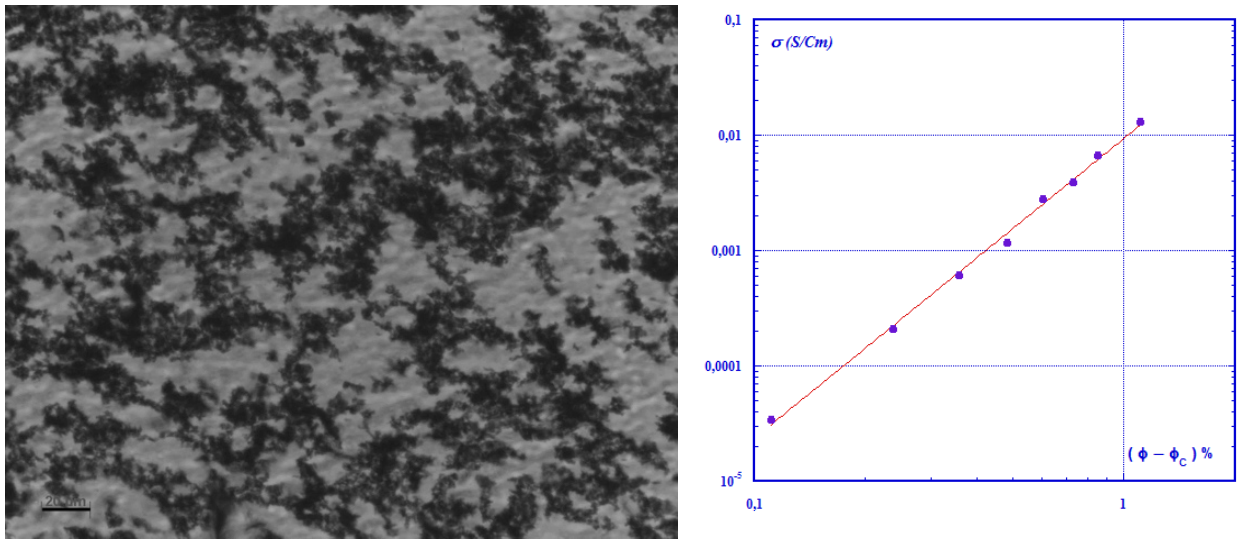

Fig. 1. a) Higher magnification (X40) of $1 \mathrm{wt} \%$ cured silicone- printex XE2 revealing the fractal Carbon Black network.

b) dc conductivity versus $\phi-\phi_{\mathrm{C}}$ of cured nanocomposites of Printex XE2 in silicone rubber. Scaling exponent deduced from power law fitting is about $\mu \cong 2,6$ with fitting accuracy $\mathrm{R}=99,89 \%$. The percolation threshold is estimated to be $\phi_{\mathrm{C}} \cong 0.15 \%$

\subsection{Effect of temperature on conductivity: variable range hopping on a fractal}

The hopping probability between two sites $i$ and $j$ decays exponentially with their separating distance due to Anderson localization. Assuming in addition, that hopping sites are homogenously distributed and that the density of states (DOS) at the fermi level $g\left(\varepsilon_{F}\right)$ is constant, Mott [39] proposed a mathematical expression for the decrease of electric conductivity at relatively low temperature in glassy systems: the Variable Range Hopping model (M-VRH)

$$
\begin{gathered}
\sigma_{D C}(T, \phi, F) \cong \sigma_{0}(\phi, F) \cdot \exp -\left(T_{0} / T\right)^{m} \\
m=1 /(d+1) \text { and } k_{B} T_{0} \simeq \alpha^{d} / g\left(\varepsilon_{F}\right)
\end{gathered}
$$

The exponent $m$ of the stretched exponential is sometimes written $\gamma$, we use here $m$ to distinguish it from critical exponents used in percolation and critical phenomena. The $\mathrm{m}$ exponent in the conduction process by VRH is simply the inverse of the total dimension of the "range" here $\left(\mathrm{d}\right.$ space +1 energy. Below the typical temperature $\mathrm{T}_{0}$, charge carriers have to optimize their hopping range given the available energy and vacant sites. The average hoping distance increases when lowering temperature as: $R_{h} \sim\left(T_{0} / T\right)^{m}$. In the case of hopping on a fractal structure, one can let the expression of Mott's exponent as simple as it is and only rescale the dimension $\mathrm{d}$ to a more generalized dimension $\tilde{d}=d_{f} / \zeta(\kappa+1)$ where we changed the assumption for the sake of generality to include : a fractal distribution of the hoping sites rather than a uniform distribution, a super-localization of the wave function $\psi(r) \sim \exp -(\alpha r)^{\zeta}$, and a density of state dependence $g(\varepsilon) \sim \varepsilon^{\kappa}$ around the fermi level. In this general case $m=1 /(\tilde{d}+1)$. Figure 2 a gives the measured DC conductivity during cooling and heating of a sample with $1,5 \mathrm{wt} \%$ (phi $=0,75$ ). Upon cooling down, we first observed change (at $\mathrm{T}=-93{ }^{\circ} \mathrm{C}$ ) in conductivity indicating the partial crystallization of the polymeric matrix. When heating, the resistance of the sample has more pronounced change around the melting point. Although these effects around theses characteristic temperatures of the polymeric matrix are important and could be used for the development of certain sensors applications making use mainly of the notable change of conductivity around the melting point but they remain out of the scope of this work. Our intension in this work is to study the conduction process and it's correlation to the structural properties of the CB network. Below $\mathrm{T}=70 \mathrm{~K}$, one notes that resistivity increases dramatically (for these reasons, sample dimensions should be chosen in such way that the resistance stay in the measurable range). At very low temperature one could check the conduction process of the $\mathrm{CB}$ network. Plotting the conductivity versus the temperature with an adequate exponent in order to find a straight line as shown in Figure 2b, one can estimate from the curve adjustment Mott's exponent $\mathrm{m}=0,65$ and $T_{0} \cong 64 \mathrm{~K}$. From Mott's exponent one can deduce a generalized dimension $\tilde{d}=(1-m) / m \cong 0,54$ which obviously can be explained only when considering VRH on a fractal. 

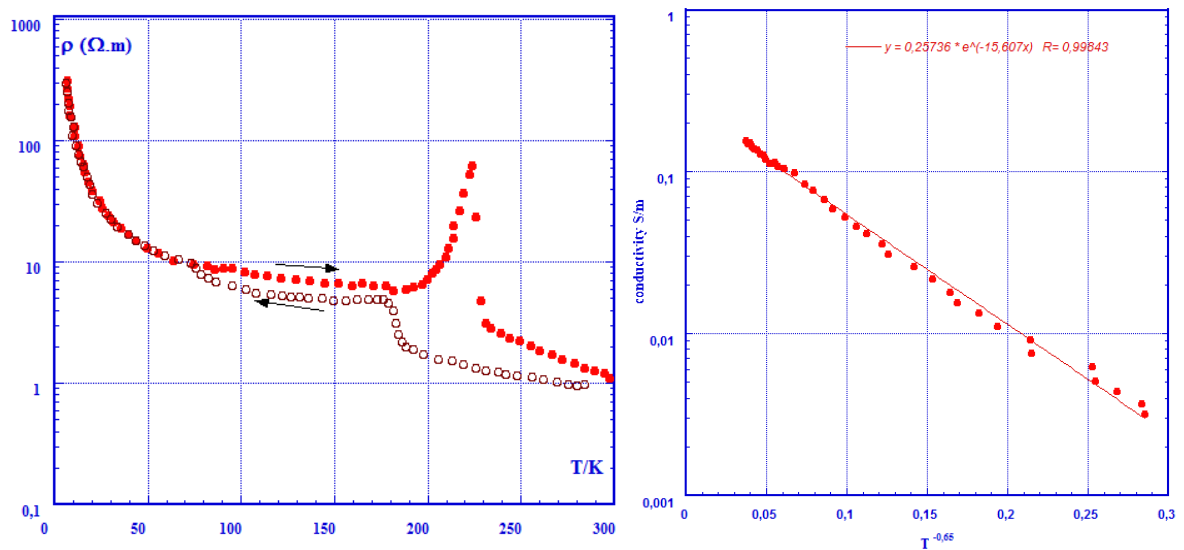

Fig. 2: a) Low temperature dc resistivity during cooling and heating $(3 \mathrm{~K} / \mathrm{mn})$ of cured samples with 3 wt $\%$ cured silicone- printex XE2

b) Typical Mott fitting of dc conductivity of the same sample as in figure $3 \mathrm{a}$ for temperature below $70 \mathrm{~K} . m=0,65$ and $\mathrm{T}_{0}=64 \mathrm{~K}$ with fitting accuracy $\mathrm{R}=99,84 \%$.

\subsection{Effect of the field strength: non ohmic conduction}

At room temperature, non - Ohmic conduction occurs gradually at applied electric field above $10^{4} \mathrm{~V} / \mathrm{m}$. Nevertheless, this departure from linearity behaviour is earlier at lower filler content as well as at lower temperature. We focused in this work on the behaviour of the non-linearity when lowering temperature.

Figure 3a shows the measured electric conductivity at different temperature when the voltage (or applied electric field) increases for sample with $1,5 \mathrm{wt} \%$ or $0,75 \%$ volume fraction of CB. We plotted $\sigma(\mathrm{V}, \mathrm{T}) / \sigma_{0}(0, \mathrm{~T})$ rather than simply the conductivity $\sigma(\mathrm{V}, \mathrm{T})$ versus the applied voltage in order to show clearly how the onset voltage of nonlinearity is temperature dependent. Let's consider that nonlinear behaviour starts when we deviate from the ohmic conductivity by $10 \%$.

$\mathrm{V}_{0}$ is the onset voltage of non-linearity defined by the former relation. Replotting $\sigma(\mathrm{V}, \mathrm{T}) / \sigma_{0}(0, \mathrm{~T})$ versus $\mathrm{V} / \mathrm{V}_{0}$ which is the same as $\mathrm{F} / \mathrm{F}_{0}$ in term of electric field, one find out that all curves superpose in a unique master curve as depicted in figure $3 \mathrm{~b}$. The onset voltage or field scales with temperature with and exponent $X_{\mathrm{T}}=1,2$. The scaling exponent of the onset linearity with "zero field conductivity" is about $\mathrm{x}_{0}=0,63$ and could be expressed $8 * 0,08$ as proposed by Nandi. The physical origin of this quantified scaling exponent is still under investigation. If one accepts that when increasing the field strength new conduction path are activated. Adjusting the hole curve at T=15 $\mathrm{K}$ with stretched exponential as suggested Jana et al.[12] we could find in deed the same $\mathrm{m}=0,64$ indicating that at this low temperature the field is responsible of charge carrier hopping. However, for higher temperature the adjustment of curves could not give the Mott exponent unless we select data at relatively high field. This could be an experimental evidence about the existence of activation less regime at relatively high field.
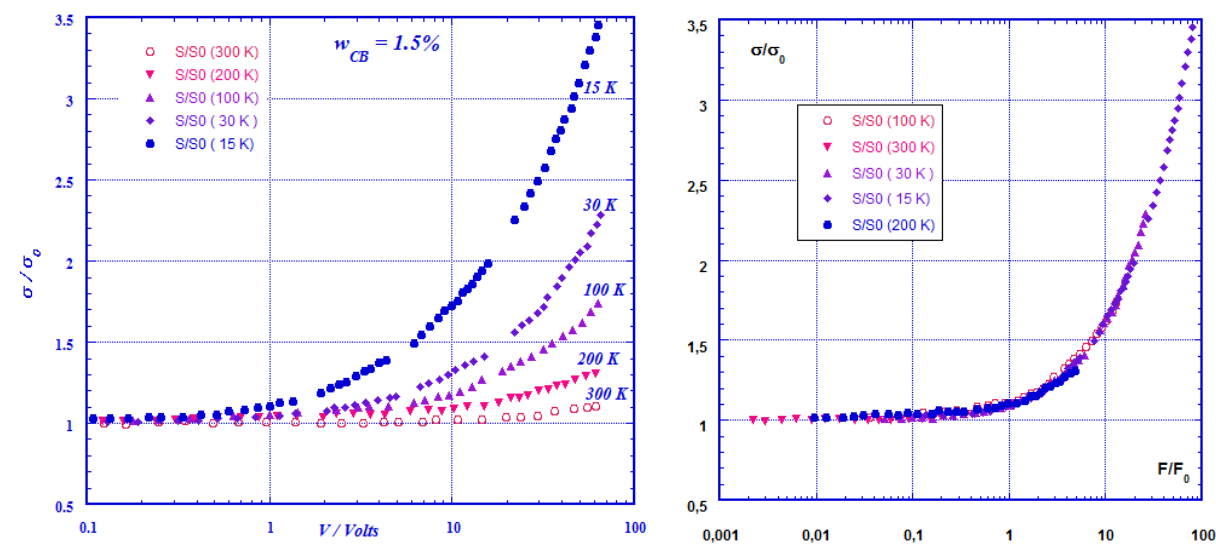

Fig. 3: a) Rescaled dc conductivity $\sigma / \sigma_{0}$ at different temperature of cured samples with $1,5 \mathrm{wt} \%$ cured siliconeprintex XE2 nanocomposites versus the applied voltage 
b) Master curve obtained when plotting $\sigma / \sigma_{0}=f\left(F / F_{0}\right)$ for cured samples with $1,5 \mathrm{wt} \%$ cured silicone- printex XE2 at different temperature.

\subsection{Effect of curing rate on surface morphology and conductivity}

A skin layer made of pure silicone is often formed when curing slowly the rubber matrix. The gravitation is not responsible for such settling down of $\mathrm{CB}$ aggregate since the skin forms even when curing the samples with gravitation field going from the bottom to the top. The origin of the skin formation is then linked to surface forces as stated by many recent works concerning micro and nanoparticles at fluid-fluid interfaces see for example [1315] for a review. Figure $4 \mathrm{a}$ shows $1 \mathrm{wt} \%$ and $5 \mathrm{wt} \% \mathrm{CB}$ samples with two different curing rates fast $(\mathrm{F})$ and slow (S). The tuning of the curing rate was achieved by the addition of an inhibitor to the curing reaction (hydrosililation), in our case 1-ethynyl-1-cyclohexanol. For fast cured sample no inhibitor was used while for slow curing sample the concentration of the inhibitor was about $0,5 \mathrm{wt} \%$.

The analysis of cross-section profile of AFM picture (figure $4 \mathrm{~b}$ ) revealed that the typical height of the immerged part of an aggregate does not exceed $50 \mathrm{~nm}$ while the perturbation parallel to the interface by the presence of immerging part of aggregate is much bigger as described by the width of half high about $200 \mathrm{~nm}$ depicted in figure 4b. In addition, we should keep in mind that the immerging part of aggregates are part of a colloidal network spanning the whole system and that the constitutive unit of this colloidal network is not a carbon black particle with typical size about a $=30 \mathrm{~nm}$ but an agglomerates with size ranging between 100-300 $\mathrm{nm}$. One has to focus first on the excess of free energy due to the presence of immerged/submerged parts of the aggregates. Clearly we have in our case a competition between the dynamic of desorption from the interface and the curing rate of the silicone rubber.

At a given curing rate, the number of immerging part of aggregate is higher when increasing the carbon black concentration resulting in a surface with higher roughness. For a given $\mathrm{CB}$ content, the number of immerging aggregate is decreasing with lower curing rate. In addition,

As can be seen on figure $5 \mathrm{a}$, the width at half height of immerging aggregate is twice of those in sample with fast curing rate. This could be the signature of rearrangement of aggregates due to lateral forces or inter-aggregates interactions due to capillary forces resulting from surface deformation.

In addition, it is also obvious that the bigger the aggregate are, the more stable at the interface they will be. In general, we noticed that slower curing rate gives less roughness of the surface. This means that small parts of agglomerate are instable at the interface and tend to be submerged by silicone during curing. Upper parts of aggregates experience also lateral forces tending to assemble them so that they become bigger and hence more stable at interface. If we consider the typical size of an aggregates is the correlation length $\xi$ then the reduced immersion depth will be typically $\bar{h}=1-a / \xi$ which is very close to one given the typical value of a $30 \mathrm{~nm}$ and $\xi>1 \mu \mathrm{m}$. This simply means that complete submergence of aggregates could be explained when considering a nonzero line tension on the immerging carbon black particles as depicted in figure 4 of reference [13] plotting the excess free energy versus the reduced immersion depth.
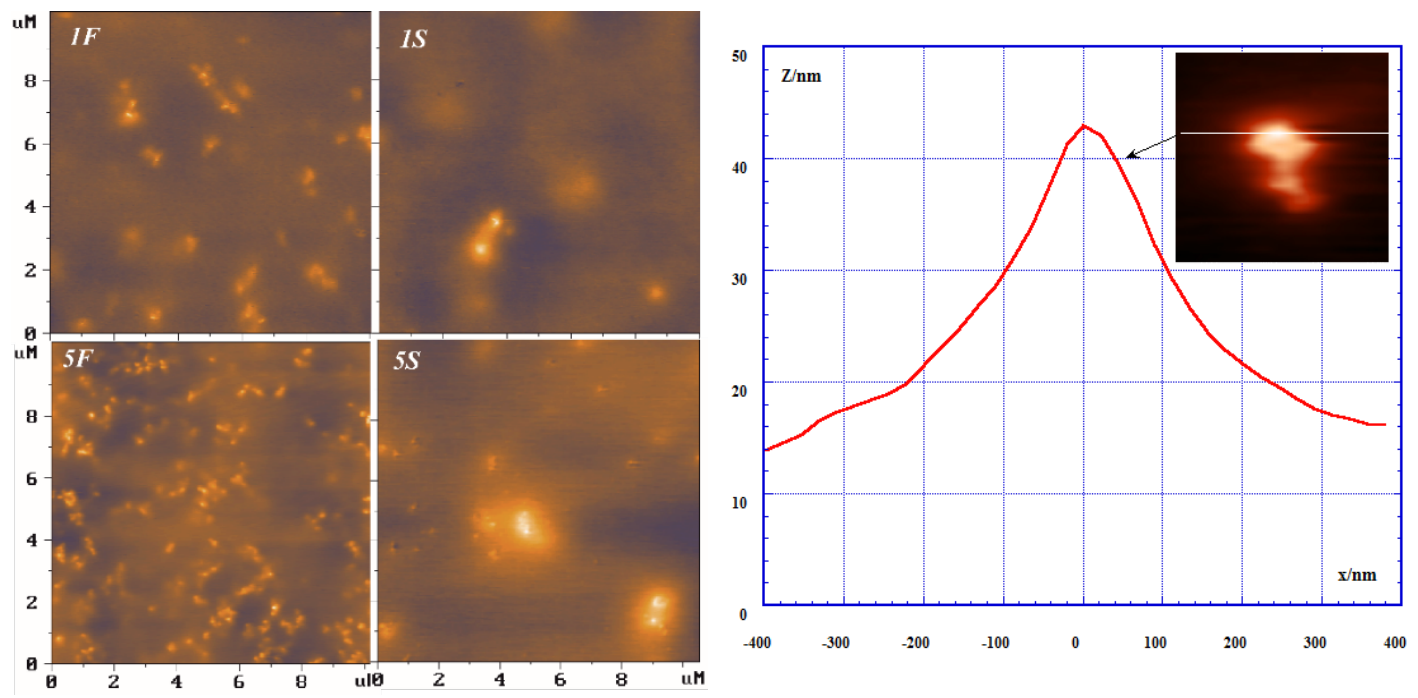
Fig. 4. a) AFM picture of silicone-printex XE2 with different $\mathrm{CB}$ content and different curing rate (1F) $1 \mathrm{wt} \%$ without inhibitor. (5F) $5 \mathrm{wt} \%$ without inhibitor (1S) $1 \mathrm{wt} \%$ with $0,5 \mathrm{wt} \%$ inhibitor (5S) $5 \mathrm{wt} \%$ with $0,5 \mathrm{wt} \%$ inhibitor. Color scale is $0-60 \mathrm{~nm}$ from dark to clear

b) A cross-section of AFM picture of an aggregate (inset picture) of a fast curing sample (without inhibitor) at CB content $1 \mathrm{wt} \%$.

\section{Conclusion}

Polymeric nanocomposites fall in the class of functional materials where one intend to make use of both filler and polymeric matrix properties, in order to achieve a given functionality. Electric conductivity is the main property one intends to improve, when introducing conductive nano filler in an insulating polymeric elastomer. In such cases, a filler network spans the systems giving a fractal path to charge carriers. The main challenge is to explain using a unified and coherent theoretical background the effects of different parameters (filler volume fraction, temperature, electric field strength...) and their correlation to structural features. VRH and percolation are the main theoretical background we used to establish structure-properties relationship in conductive CB-Silicone nanocomposites. However, one has to adapt these general theories to the particularity of our system. In fact, the low percolation threshold can be explained by the fact that we have percolation of blob of agglomerates rather than percolation of single CB particles. In such case if one admits that hard spheres has a percolation threshold about $16 \%$, the blobs should have an internal volume fraction about $1 \%$ in order to achieve the measured threshold $(0,15 \%)$. The measured scaling exponent of the dc conductivity is about 2,404 and could be connected to the measured fractal dimension if one assumes a fraction between the random walk dimension and the fractal dimension about 1,8 and not 3/2 as assumed by Alexander-Orbach. The measured fractal dimension of the carbon black network is about the estimated fractal dimension of a percolation network within the error of measure. However as stated, by Stanley [6] this alone cannot lead us to conclude that aggregation process is percolation (since even diffusion limited cluster aggregation has the same fractal dimension). The other exponents of sub structural features and dynamical behaviour confirm that percolation of already present agglomerates is behind the formation of the macroscopic filler network.

During thermal transitions of the polymeric matrix (melting-crystallization-glass transition) one could measure a notable change in conductivity and these effects could have interesting applications. Fundamental and quantitative explanations of such effects is still lacking in literature. Our attention was more oriented to the charge carrier conduction process at low temperature. One showed that VRH hopping is indeed the conduction process and found a Mott exponent about 0,64 and Mott temperature. The former Mott's exponent could be explained by extending the original Mott model into VRH on a fractal rather than a homogenous distribution of hopping sites. If in addition one assumes a super-localization exponent about then we can deduce that a non-constant density of state around the fermi level is needed to explain the measure Mott exponent. Nevertheless, more experimental investigations and evidence about super-localization and hopping on fractal structure is needed. Non-ohmic conduction occurs at an onset field of non-linearity $\mathrm{F}_{0}$ which scales with temperature as well as with the zero field limit conductivity. Clearly much more theoretical work on non-ohmic conduction process in percolating networks is needed. Curves of non-Ohmic conductivity versus the field strength could be superposed in one master curve. The surface morphology and conductivity, as measured by SPM techniques, is found to be affected by both the filler content and the curing rate of the rubber matrix. There is clearly two competing dynamics the desorption kinetics of immerging parts of aggregates and the curing rate of the rubbery matrix. Both AFM and STM confirm that the higher the curing rate and $\mathrm{CB}$ content the more roughness we have. But even at relatively high concentration compared to the percolation threshold; smooth surface is obtained with a top skin silicone layer when using slow curing rate. We attempted to interpret our results using the newly immerging field of nanoparticles at fluid interfaces. Although in our case we have flocculated suspension of nanoaggregates and not floating nanoparticles at the interface, we could find some similarity in the behaviour of immerging part of aggregates at the interface. Finally, surface property of suspension when the fluid experience a curing reaction is of major importance in coating technology and should be address theoretical works.

\section{Acknowledgements}

Dr R. NEFFATI thanks King Khalid University for funding the functional polymer group under project number 295. The Authors are grateful to Prof. Dr. Michaels, Dr. A. Alexeev, Dr. J. Loos, S. Geralds, M. Van Vleuten and R. Sturm for supportive help and fruitful discussions. 


\section{References}

1. Al-Saleh, M.H., Saadeh, W.H., and Sundararaj, U.: 'EMI shielding effectiveness of carbon based nanostructured polymeric materials: a comparative study', Carbon, 2013, 60, pp. 146-156

2. Kwon, S.K., Ahn, J.M., Kim, G.H., Chun, C.H., Hwang, J.S., and Lee, J.H.: 'Microwave absorbing properties of carbon black/silicone rubber blend', Polymer Engineering \& Science, 2002, 42, (11), pp. 2165-2171

3. Lacasse, M.-A., Duchaine, V., and Gosselin, C. Eds. 'Book Characterization of the electrical resistance of carbon-black-filled silicone: Application to a flexible and stretchable robot skin' (IEEE, 2010, edn.), pp. 48424848

4. Chen, X., Jiang, Y., Wu, Z., Li, D., and Yang, J.: 'Morphology and gas-sensitive properties of polymer based composite films', Sensors and Actuators B: Chemical, 2000, 66, (1-3), pp. 37-39

5. Liu, P., Liu, C., Huang, Y., Wang, W., Fang, D., Zhang, Y., and Ge, Y.: 'Transfer function and working principle of a pressure/temperature sensor based on carbon black/silicone rubber composites', Journal of Applied Polymer Science, 2016, 133, (7)

6. Pynn, R., and Skjeltorp, A.: 'Scaling Phenomena in Disordered Systems' (Plenum Press 1991. 1991)

7. Armin, B., and Shlomo, H.: 'Fractals and Disordered Systems' (Springer, 1996. 1996)

8. Zallen, R. 'The physics of amorphous solids' (John Wiley \& Sons, 1983. 1983).

9. Neffati, R.: 'Carbon Black-Silicone Melts Suspensions: Fractal morphology and rheological properties' to be submitted

10. Geraedts, S.: 'Pore size distribution and surface group analysis a study of two electrical grade carbon blacks', Eindhoven University of Technology, 2002

11. Alexander, S., and Orbach, R.: 'Density of states on fractals : “ fractons "', J. Phys. lett., 1982, 43, (17), pp. 625-631

12. Jana, D., and Fort, J.: 'A simple scaling approach to Mott conductivity', Physica B: Condensed Matter, 2004, $344,(1-4)$, pp. $62-65$

13. Bresme, F., and Oettel, M.: 'Nanoparticles at fluid interfaces', J Phys Condens Matter, 2007, 19, (41), pp. 413101

14. kralchevsky, P.A., and Nagayama, K.: 'Capillary interactions between particles bound to interfaces, liquid films and biomembranes, Advances in Colloid and Interface Science, 2000, 85, pp. 145-192

15. Zanini, M., and Isa, L.: 'Particle contact angles at fluid interfaces: pushing the boundary beyond hard uniform spherical colloids', J Phys Condens Matter, 2016, 28, (31), pp. 313002 


\title{
Nanoporous Carbonaceous Adsorbents for Enrichment of Ventilation Air Methane (VAM) with Methane
}

\author{
E. Brodawka, ${ }^{1,}$ M. Bałys, ${ }^{1}$ J. Szczurowski, ${ }^{1}$ L. Czepirski, ${ }^{1}$ K. Zarębska, ${ }^{1}$ P. Da Costa ${ }^{2}$ \\ ${ }^{1}$ AGH University of Science and Technology, Faculty of Energy and Fuels, Krakow, Poland \\ ${ }^{2}$ Sorbonne Université, Institut Jean le Rond D'Alembert, Paris, FRANCE
}

\begin{abstract}
In this paper new concept for the realization of the PSA cycle to enrich the mixture streams with methane was analysed. The removal of methane from VAM is the separation between methane and nitrogen and properly selected adsorbent is a key to the separation by means of the PSA process. The separation effect is based on each component's selectivity for the adsorbent material. The most promising adsorbents for VAM enrichment with methane seems to be carbonaceous adsorbents thanks to its unique porous texture with narrow micropores, large specific surface area and porosity, hydrophobic surface, various surface functional groups. The new concept of the PSA process was verified in the experimental unit. The gas mixture was fed into the column packed with the adsorbent. When the separation between components of the mixture is realized, the enriched stream on the top of the column is collected as the product. The column is regenerated by reducing the pressure to vacuum. Desorbed gas is recovered on the bottom of the column. A mathematical model was developed to optimize process variables (cycle time, pressure ratio) for the individual steps. The results of the experiment confirmed the correctness of calculations undertaken. The concentration of methane in the product (desorbed gas) increases during depressurization step from 0.3 vol.\% (methane concentration in the feed gas) to 2.0 vol. $\%$ at the end of this step. The results indicated that selected activated carbon can be successfully applied in $\mathrm{N}_{2} / \mathrm{CH}_{4}$ separation for ventilation air methane and there is still a chance for improvement in the performance of these systems and further experiments should be continued to optimize of process variables, as well as search new types of carbonaceous adsorbents.
\end{abstract}

Keywords: Pressure Swing Adsorption, nanoporous carbonaceous adsorbent, ventilation air methane, gas mixture separation

\section{Introduction}

Currently, the special interest is a separation of gaseous mixtures containing a low concentration of methane (biogas, ventilation air methane, landfill gases) which could be separated using carbonaceous adsorbents (activated carbons, carbon molecular sieves, activated carbon fibres) [1]. It is well known that methane is an anthropogenic greenhouse gas (more than 20 times as potent as $\mathrm{CO}_{2}$ ), which imposes the most significant influence on the global warming issue. Ventilation air methane (VAM) is the largest source of coal mine methane (CMM) emissions. Most coal mines around the world discharge ventilation air methane (VAM) into the air directly. In Poland, around 600 million $\mathrm{m}^{3} \mathrm{CH}_{4}$ was emitted to the atmosphere with mine ventilation air [2]. Methane emitted from coal mines ventilation systems into the air is the most difficult to use as an energy source, because of the low methane concentration (typically 0.3 to $0.5 \mathrm{vol} \%$ in the Polish mine workings), large airflows and variable, both flow and concentration. Adsorption-based processes including Pressure Swing Adsorption (PSA) are potential technologies that could be used to recover methane from vent streams and enrich the methane to a concentration level [3] that could be practically used as a supplement fuel e.g. in internal combustion engines and gas turbines or as a principal fuel in thermal or catalytic flow-reversal oxidizers or lean-burn gas turbines [4]. In this work new concept for the realization of the PSA cycle to enrich the mixture streams with methane was analysed, as well as the texture parameters of carbon materials were measured.

\section{Experimental}

\subsection{Materials}

The used adsorbents were five non-modified, commercial, nanoporous carbonaceous adsorbents, namely AC1, $\mathrm{AC} 2, \mathrm{AC} 3, \mathrm{AC} 4$ and AC5. Textural characteristics of the samples, obtained by $\mathrm{N}_{2}$ isotherms at $77 \mathrm{~K}$ measured by 
the automatic volumetric sorption analyser (Autosorb-1C, Quantachrome, USA), are shown in Table 1. Fig. 1 presents the $\mathrm{N}_{2}$ isotherms on the selected samples. The specific surface area $\left(\mathrm{S}_{\mathrm{BET}}\right)$ was calculated by the Brunauer-Emmett-Teller (BET) method. The calculation of mesopore size $\left(\mathrm{V}_{\text {mes }}\right)$ and average diameter of mesopores was carried out by applying the BJH model to the adsorption branch of the isotherm. The micropore volume $\left(\mathrm{V}_{\text {mic }}\right)$ and an average width of micropores were obtained by Dubinin-Radushkevich method, and pore size distribution was determined by Density Functional Theory (DFT) method.

Table 1: Porous texture of activated carbons

\begin{tabular}{|c|c|c|c|c|c|}
\hline sample & AC1 & AC2 & AC3 & AC4 & AC5 \\
\hline BET surface area, $\mathrm{S}_{\text {BET }}\left[\mathrm{m}^{2} / \mathrm{g}\right]$ & 2225 & 2460 & 1171 & 723 & 1118 \\
\hline Micropore volume, $\mathrm{V}_{\text {mic }}\left[\mathrm{cm}^{3} / \mathrm{g}\right]$ & 0.76 & 0.81 & 0.38 & 0.28 & 0.42 \\
\hline Mesopore volume, $\mathrm{V}_{\text {mes }}\left[\mathrm{cm}^{3} / \mathrm{g}\right]$ & 0.84 & 0.68 & 0.16 & 0.02 & 0.06 \\
\hline Average diameter of mesopores $[\mathrm{nm}]$ & 2.11 & 2.25 & 2.24 & 2.11 & 2.18 \\
\hline Average width of micropores $[\mathrm{nm}]$ & 1.64 & 1.69 & 1.36 & 1.01 & 1.15 \\
\hline
\end{tabular}

Fig.2 presents differential pore volume distributions of the sample $\mathrm{AC} 1$ calculated from (a) $\mathrm{N}_{2}$ adsorption isotherm at $77 \mathrm{~K}$, (b) $\mathrm{CO}_{2}$ adsorption isotherm at $273 \mathrm{~K}$ and $\mathrm{N}_{2}+\mathrm{CO}_{2}$ adsorption isotherm using the NLDFT model.

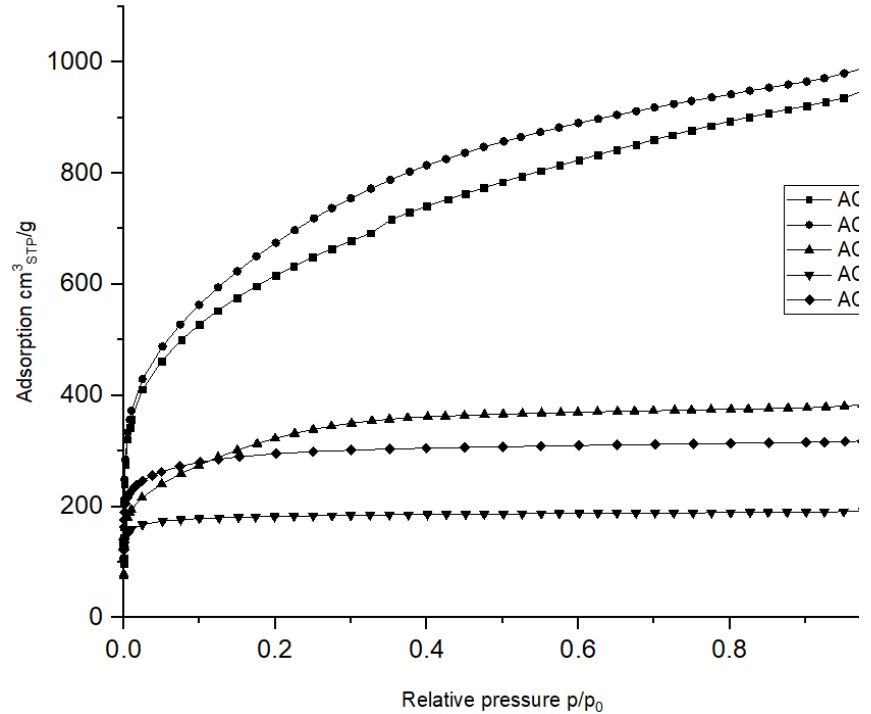

Fig.1: Isotherms of $\mathrm{N}_{2}$ at $77 \mathrm{~K}$ on the samples used in the present study.

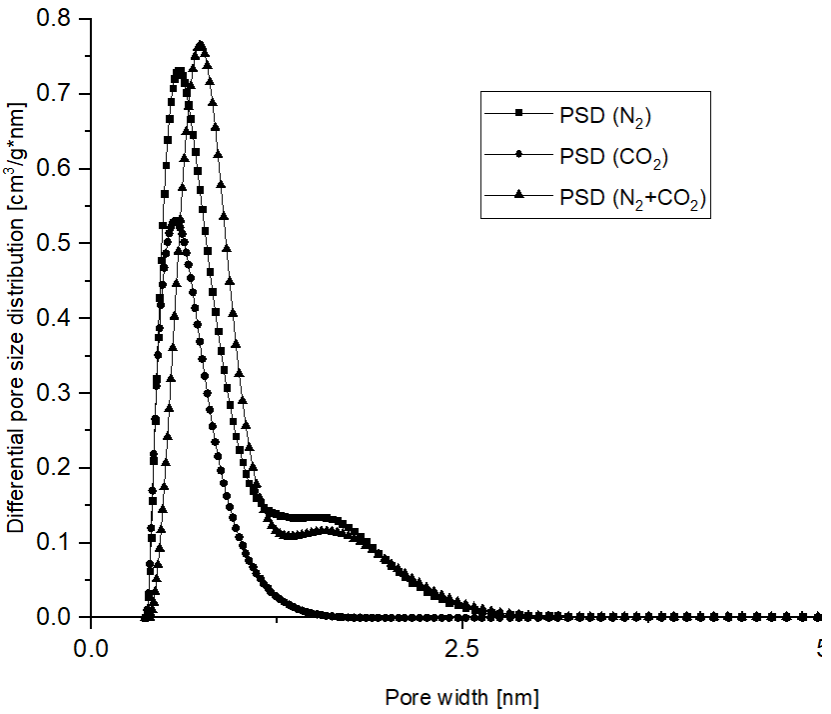

Fig.2: Differential pore volume distributions of the sample AC5.

\subsection{Measurement of adsorption equilibrium isotherms}

The automatic volumetric sorption analyser (Autosorb-1C, Quantachrome, USA) was used to measure the adsorption equilibrium isotherms of $\mathrm{CH}_{4}$ and $\mathrm{N}_{2}$ on the selected carbon materials. Pure $\mathrm{CH}_{4}$ and $\mathrm{N}_{2}$ adsorption isotherms on materials were measured at $293 \mathrm{~K}$ in a pressure range of $0-101,325 \mathrm{kPa}$ after $12 \mathrm{~h}$ degassing at $423 \mathrm{~K}$ and deep vacuum. Fig. 3 presents isotherms of $\mathrm{CH}_{4}$ and $\mathrm{N}_{2}$ on the sample AC5.

In order to describe the equilibrium isotherms of methane and nitrogen, Henry's and Langmuir's adsorption isotherms were used [5]. Those isotherms can be written as follow:

$$
\begin{gathered}
\Theta=K_{H} \cdot p \\
\Theta=\frac{m \cdot b \cdot p}{1+b \cdot p}
\end{gathered}
$$

where:

$K_{H}-$ Henry's constant, 
$m, b$ - Langmuir's constants.

As a measure of separation possibility of adsorbent material we introduce separation factor defined as [5]:

$$
S=\frac{K_{H}^{C H_{4}}}{K_{H}^{N_{2}}}
$$

The constants of Henry's and Langmuir's isotherms and separation factors are summarized in Table 2 for each sample.

Table 2: Fitting parameters of Henry and Langmuir isotherms for adsorption equilibrium isotherms of single $\mathrm{CH}_{4}$ and $\mathrm{N}_{2}$ and separation factor for each sample.

\begin{tabular}{|c|c|c|c|c|c|}
\hline sample & AC1 & AC2 & AC3 & AC4 & AC5 \\
\hline \multicolumn{5}{|c|}{ methane, adsorption isotherm at 293K } \\
\hline$m$ & 3.080 & 3.124 & 1.497 & 1.848 & 2.511 \\
\hline $\mathrm{a}$ & $4.02 \mathrm{E}-04$ & $3.73 \mathrm{E}-04$ & $7.50 \mathrm{E}-04$ & $1.44 \mathrm{E}-03$ & $1.12 \mathrm{E}-03$ \\
\hline $\mathrm{K}_{\mathrm{H}}$ & $1.24 \mathrm{E}-03$ & $1.16 \mathrm{E}-03$ & $1.12 \mathrm{E}-03$ & $2.66 \mathrm{E}-03$ & $2.82 \mathrm{E}-03$ \\
\hline $\mathrm{7}$ nitrogen, adsorption isotherm at 293K \\
\hline $\mathrm{m}$ & 0.701 & 0.622 & 0.459 & 0.687 & 1.055 \\
\hline $\mathrm{a}$ & $5.06 \mathrm{E}-04$ & $5.82 \mathrm{E}-04$ & $7.43 \mathrm{E}-04$ & $8.27 \mathrm{E}-04$ & $6.32 \mathrm{E}-04$ \\
\hline $\mathrm{K}_{\mathrm{H}}$ & $3.55 \mathrm{E}-04$ & $3.62 \mathrm{E}-04$ & $3.41 \mathrm{E}-04$ & $5.68 \mathrm{E}-04$ & $6.67 \mathrm{E}-04$ \\
\hline \multicolumn{7}{|c|}{ separation factor } \\
\hline
\end{tabular}

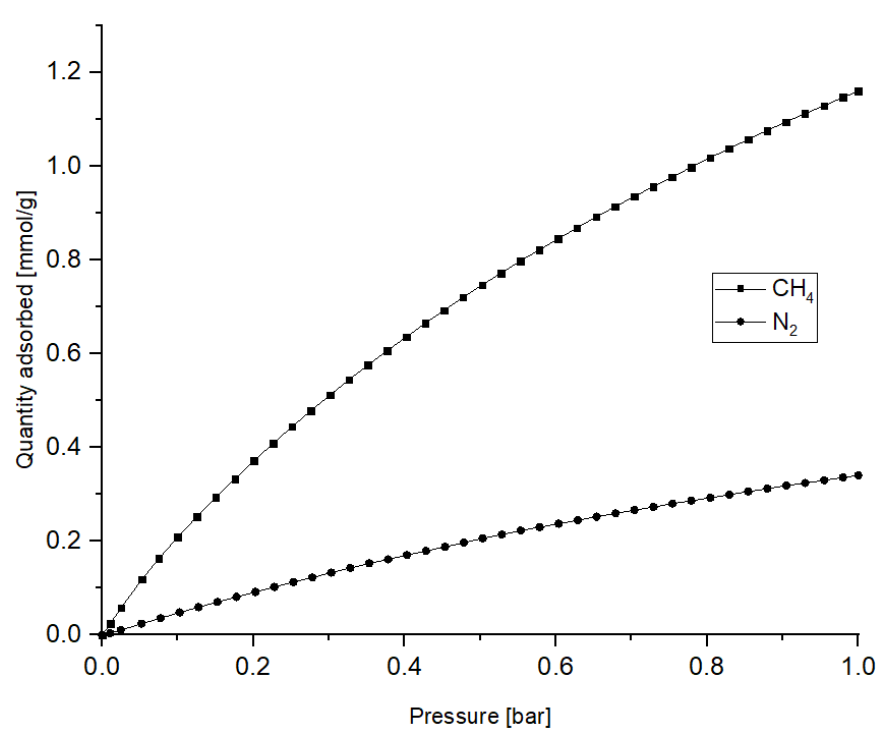

Fig. 3: Isotherms of $\mathrm{CH}_{4}$ and $\mathrm{N}_{2}$ at $293 \mathrm{~K}$ on the sample AC5.

\subsection{PSA experiments}

The new concept of the PSA process was verified in the one-bed experimental unit (Fig.4). A three-step cycle was studied comprising pressurization to the highest pressure (1bar), feed with simultaneous adsorption, countercurrent blowdown with simultaneous desorption. The blowdown step was performed in a deep vacuum. Feed gas containing $0.3 \mathrm{vol} . \% \mathrm{CH}_{4}$ and $99.7 \mathrm{vol} . \% \mathrm{~N}_{2}$ was introduced to the packed bed with AC5 adsorbent during the first two steps. According to several tests, the concentration of methane increases from 0.3 vol.\% (gas feed) to 2.0 vol.\% at the end of the desorption step. Average methane concentration in product gas was around $1 \%$. 


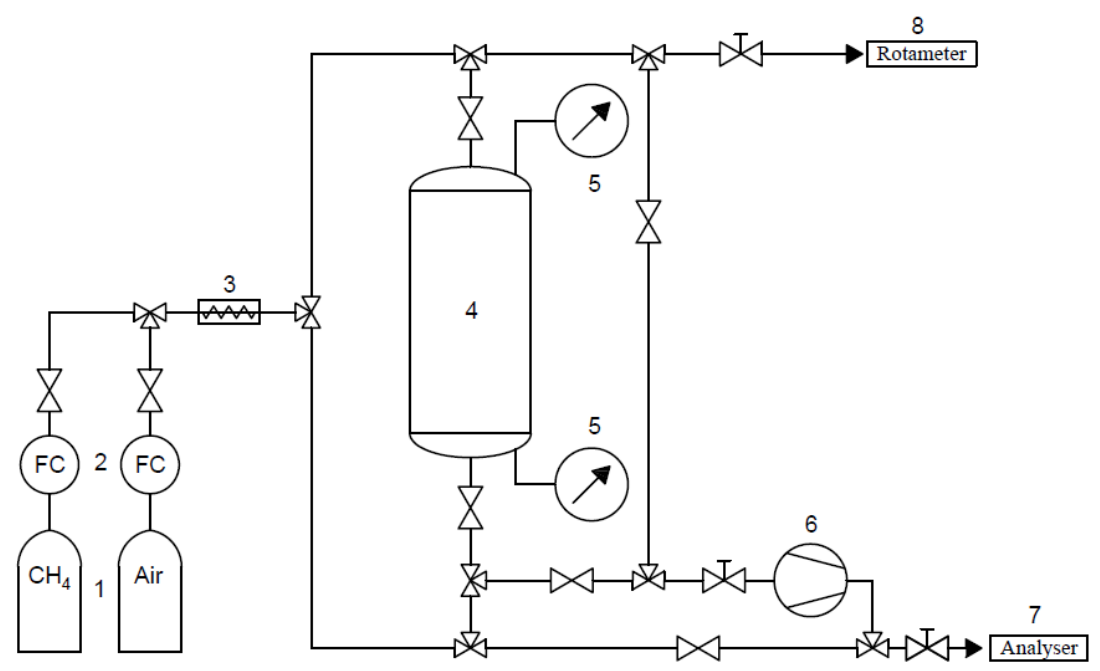

Fig. 4: Scheme of the adsorption separation unit. 1 - gas bottle, 2 - mass flow controller, 3 - mixer, 4 - adsorption column, 5 - pressure sensor, 6 - vacuum pump, 7 - gas analyser, 8 - rotameter. Source: own work

\section{Conclusion}

In this work, we propose a novel concept of an adsorption-based process for VAM enrichment. Results show a pressure swing adsorption process could be used to recover methane from VAM and enrich to the industrial requirements. Micropore carbonaceous adsorbents thanks to its unique porous texture, large specific surface area and porosity seems to be the most promising adsorbents for VAM enrichment with methane. Although experimental results obtained from the four-step one-bed PSA process demonstrate that the low-concentration methane can be enriched, methane purity in product gas is still limited by the $\mathrm{CH}_{4} / \mathrm{N}_{2}$ selectivity on carbon materials and process conditions. Further work will be carried out to improve the PSA process with a search of new types of adsorbents (activated carbons, carbon molecular sieves, activated carbon fibers, activated carbon monoliths), as well as optimization of process variables.

\section{Acknowledgements}

Financial support for this work was provided by AGH University of Science and Technology framework No. 16.16.210.476.

\section{References}

1. D. Qu, Y. Yang, K. Lu, L. Yang, P. Li, J. Yu, A. M. Ribeiro. A. E. Rodrigues, "Microstructure effect of carbon materials on the low-concentration methane adsorption separation from its mixture with nitrogen" Adsorption, vol. 24, no. 4, pp. 357-369, 2018.

2. S. Nawrat, Z. Kuczera, R. Łuczak, P. Życzkowski, S. Napieraj, K. Gatnar, "Utylizacja metanu z pokładów węgla w polskich kopalniach podziemnych", Uczelniane Wydawnictwo Naukowo-Dydaktyczne - AGH, Kraków, 2009.

3. Y. Liu, Y. Li, W. Liu, X. Yang, H. Yang, Y. Meng, "Ventilation air methane recovery by pressure swing adsorption", 5th Int. Conf. Bioinforma. Biomed. Eng., 2011.

4. M. Bałys, J. Szczurowski, L. Czepirski, "Adsorption technology for Ventilation Air Methane enrichment", Selected issues related to mining and clean coal technology, Monograph, pp. 253-257, 2016.

5. S. Sircar, "Adsorption”, Encyclopedia of Chemical Processing, 2006, Taylor\&Francis, pp. 25-39. 


\title{
Comparison of the oxygen gas and UV barrier properties of nano $\mathrm{ZnO}$ coated PET and PHBHHx packaging films via ultrasonic spray coating
}

\author{
Mohsin Abbas ${ }^{1 *}$, Mieke Buntinx ${ }^{1}$, Wim Deferme ${ }^{2,3}$, Naveen Reddy ${ }^{1}$, Roos Peeters $^{1}$ \\ ${ }^{1}$ Hasselt University, IMO-IMOMEC, Packaging Technology Center, \\ Science Park 27, 3590 Diepenbeek, Belgium, mohsin.abbas@uhasselt.be \\ ${ }^{2}$ Hasselt University, IMO-IMOMEC, Functional Materials Engineering Group, \\ Science Park 1, 3590 Diepenbeek, Belgium \\ ${ }^{3}$ IMEC vZw-Division IMOMEC, \\ Science Park 1, 3590 Diepenbeek, Belgium
}

\begin{abstract}
Synthetic polymers are widely used due to their low cost and high performance. On the other hand, these synthetic polymers have a negative impact on the environment because most of them are non-biodegradable and they cannot be decomposed by micro-organisms or bacteria. Bioplastics are more environmentally friendly due to their biobased origins and/or biodegradability. However, their barrier properties are not optimal for food packaging applications. In the past, nanoparticles (NPs) of zinc oxide $(\mathrm{ZnO})$, titanium dioxide $\left(\mathrm{TiO}_{2}\right)$ and aluminum oxide $\left(\mathrm{Al}_{2} \mathrm{O}_{3}\right)$ have been used in matrices of various bio(polymers) through different processing methods such as extrusion blow moulding, solvent casting etc., to optimize the barrier properties. Wet-chemical deposition, physical vapor deposition (PVD) and chemical vapor deposition (CVD) are the prominent deposition techniques of these nanoparticles. In this research, ultrasonic spray coating, a wet-chemical technique - has been adopted to deposit ZnO NPs on commercial poly(ethylene terephthalate) PET film and self-made poly(3-hydroxybutyrateco-3-hydroxyhexanoate) PHBHHx materials. These materials were coated with $2.5 \mathrm{wt} \% \% \mathrm{ZnO}$ NPs solution under optimized conditions of the spray coater. For 50x ZnO NPs coated PET substrates, oxygen transmission rate (OTR) results showed a slight decrease of 5\% in OTR value and no decrease in OTR was observed for 50x $\mathrm{ZnO}$ NPs coated PHBHHx substrates. Moreover, a significant decrease in UV barrier properties for $3 \mathrm{x}$ and $5 \mathrm{x} \mathrm{ZnO}$ NPs coated PET and PHBHHx substrates was attained at 370 and $390 \mathrm{~nm}$ respectively.
\end{abstract}

Keywords: poly(ethylene terephthalate) (PET), poly(3-hydroxybutyrate-co-3-hydroxyhexanoate) (PHBHHx), ultrasonic spray coating, oxygen transmission rate.

\section{Introduction}

Zinc oxide nanoparticles (ZnO NPs) have been progressively studied in many research areas due to their unique functional properties. These nanoparticles have also been used in packaging materials to enhance their barrier properties like oxygen $\left(\mathrm{O}_{2}\right)$, carbon dioxide $\left(\mathrm{CO}_{2}\right)$ and water vapor barrier properties. In this study, we have used two different kind of materials, a synthetic polymer - Polyethylene terephthalate (PET) and a biopolymer - Poly(3hydroxybutyrate-co-3-hydroxyhexanoate) (PHBHHx). PET is a highly demanding packaging material and PHBHHx is an emerging biopolymer with biodegradable characteristics. Deposition methods such as physical vapor deposition (PVD), chemical vapor deposition (CVD) and wet-chemical method have been used to deposit different nanoparticles on different (bio)polymers. In wet-chemical methods, the ultrasonic spray coating is a versatile technique due to less material consumption, creation of relatively small droplets of a few microns in combination with rapid evaporation of the solvents [1] and uniform coating by optimizing its operating parameters. The aim of this research was to optimize the ultrasonic spray coating process regarding the deposition of ZnO NPs on PET and PHBHHx substrates and then to study the oxygen and UV barrier properties of these coated substrates.

\section{Experimental}

\subsection{Materials}

$\mathrm{ZnO}$ nanoparticles - 20 wt.\% dispersion in water, Isopropanol (IPA), Polyvinyl alcohol (PVA) and De-ionized water (DI water) were used. The concentration of ZnO NPs dispersion was optimized to $2.5 \mathrm{wt} . \%$ for spray coating experiments. The substrates used for ZnO NPs coating were commercial grade PET foil with 125 micron thickness 
and self-made PHBHHx samples with $\sim 0.5 \mathrm{~mm}$ thickness. Self-made PHBHHx samples were made through its granulates by using Plate Press, which was kindly provided by KU Leuven, Belgium. PET foil and PHBHHx samples were cut into dimensions of $3.5 \mathrm{~cm} \times 3.5 \mathrm{~cm}$. PET samples were cleaned with soapy water, DI water $(2 \mathrm{x})$ under 15 min sonication for each step, followed by soaking these samples in acetone for few seconds. Then these samples were immersed in IPA solution for 15 min sonication. Finally, these samples were dried in air with Nitrogen $\left(\mathrm{N}_{2}\right)$ gun.

\subsection{Methods}

To deposit ZnO NPs on PET and PHBHHx samples, an ultrasonic spray coater from Sono-Tek corporation (USA), equipped with an AccuMist ${ }^{\mathrm{TM}}$ system of ultrasonic spraying nozzle, was used. An ultrasonic spray coater works on the principle of ultrasonic atomization. AccuMist ${ }^{\mathrm{TM}}$ nozzle, as shown in Fig. 1 [2], operates by transforming high frequency sound waves into mechanical energy that is transferred into a liquid, creating standing waves at the tip of the nozzle [3]. Consequently, the liquid is fragmented into a fine mist of micron sized droplets at the atomizing surface of the nozzle.

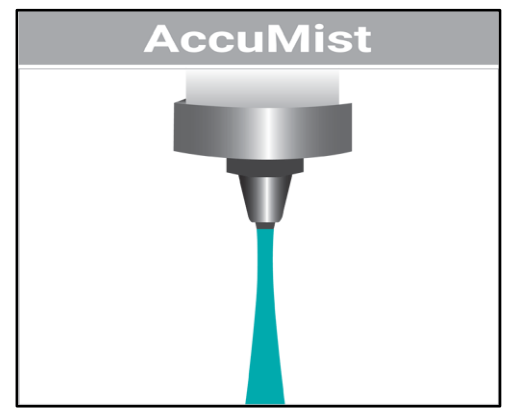

Fig. 1: Ultrasonic spray shaping of an AccuMist ${ }^{\mathrm{TM}}$ nozzle

A FEI Quanta 200F - scanning electron microscope (SEM), USA was used to determine the surface morphology of the ZnO NPs coated PET and PHBHHx samples. To measure the oxygen transmission rates $\left(\mathrm{OTR}-\mathrm{cc} / \mathrm{m}^{2}-\right.$ day) of neat and ZnO NPs coated samples, a Mocon Ox-Tran ${ }^{\circledR}$ was used. The samples for OTR tests were prepared in the form of aluminum masks of surface area of $5 \mathrm{~cm}^{2}$. The operating conditions used for the OTR tests were $23^{\circ} \mathrm{C}$ and $0 \%$ relative humidity (RH) respectively. UV barrier properties of ZnO NPs coated PET and PHBHHx substrates were determined by Cary 5000 UV-Vis-NIR spectrometer.

\section{Results and Discussion}

The ultrasonic spray coater was optimized with its operating parameters for both substrates - pump flow rate $(0.1$ $\mathrm{ml} / \mathrm{min})$, path speed $(10 \mathrm{~mm} / \mathrm{sec})$, generator power $(2.5$ watts $)$, nozzle to substrate distance $(75 \mathrm{~mm})$, hot plate temperature $\left(30^{\circ} \mathrm{C}\right)$ and the amount of $\mathrm{ZnO}$ NPs coated layers (50x). After $\mathrm{ZnO}$ NPs deposition, top side and cross-sectional SEM images of 50x ZnO NPs coated PET and PHBHHx substrates were obtained, as shown in Figure 2 (a) \& (b) and Figure 3 (c) \& (d), respectively.
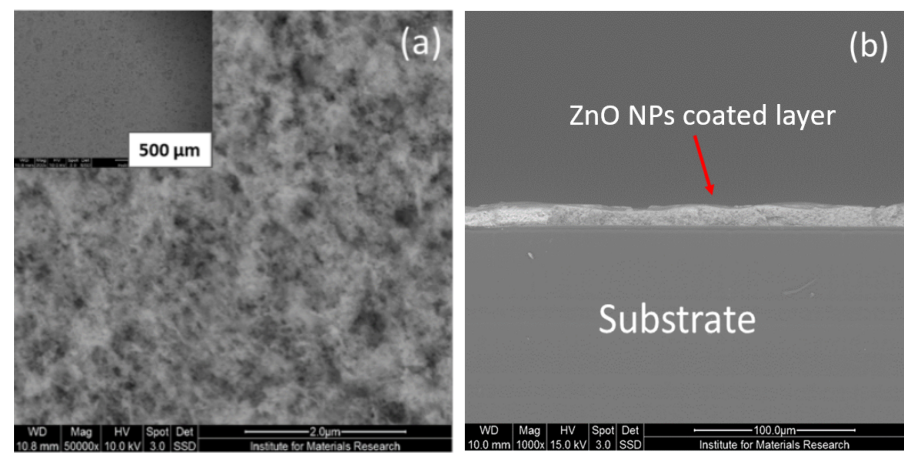

Fig. 2: (a) Top side SEM image, (b) Cross-sectional SEM image of 50x ZnO NPs coated PET substrate 

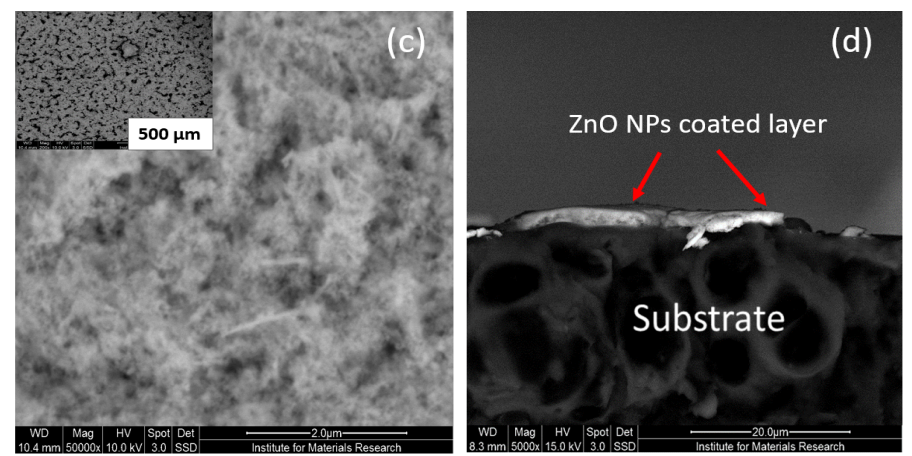

Fig. 3: (c) Top side SEM image, (d) Cross-sectional SEM image of 50x ZnO NPs coated PHBHHx substrate

The OTR tests of neat and 50x ZnO NPs coated PET and PHBHHx substrates were repeated three times. The results showed a slight decreasing trend in OTR value of 50x ZnO NPs coated PET samples. The OTR values of neat and coated PET samples were obtained as $12.2 \pm 0.3$ and $11.6 \pm 0.2 \mathrm{cc} /\left[\mathrm{m}^{2}\right.$-day], respectively. This slight decrease of $5 \%$ in OTR value could be associated to the reason of availability of a less tortuous path to small oxygen molecules to pass through the ZnO NPs coated layer. In case of PHBHHx substrates, the OTR values of neat and 50x ZnO NPs coated samples were unchanged, as $14.5 \pm 0.3$ and $14.5 \pm 0.4 \mathrm{cc} /\left[\mathrm{m}^{2}\right.$-day], respectively. No decrease in OTR values for ZnO NPs coated PHBHHx substrate was due to its hydrophobic behaviour, which lead to less adhesion of $\mathrm{ZnO}$ NPs particles to its surface.

For UV tests, 3x and 5x ZnO NPs coated PET and PHBHHx substrates were prepared. The UV-vis spectra was obtained in terms of wavelength (nm) vs. \% transmission (\%T). In Graph 1, for 3x and 5x ZnO NPs coated PET substrates, ZnO NPs absorption peak was obtained at $370 \mathrm{~nm}$ and Graph 2 showed the absorption peak at $390 \mathrm{~nm}$ for $3 \mathrm{x}$ and $5 \mathrm{x} \mathrm{ZnO}$ NPs coated PHBHHx substrates. Both results are in accordance with the literature for absorption range of ZnO NPs in the UV region [4]. For ZnO NPs coated PET substrates, a significant decrease in $\%$ transmission was observed as compared to neat PET substrate. In case of PHBHHx substrates, less sharp peaks were observed that could be due to its more thickness and less transparency, as compared to commercial grade PET foil.

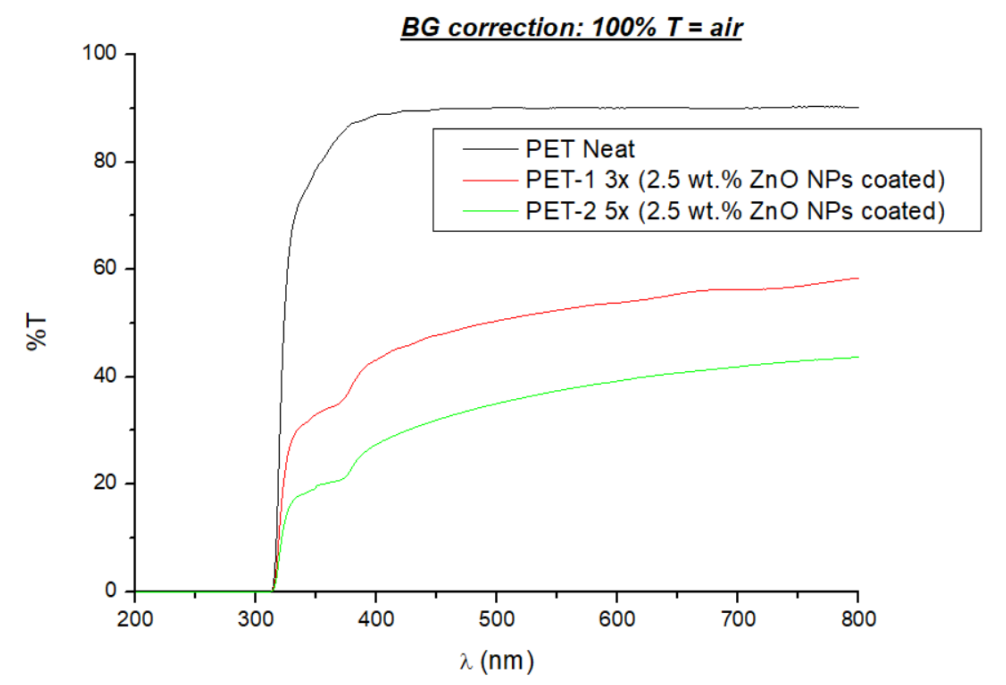

Graph 1: UV-vis spectra of neat, 3x and 5x ZnO NPs coated PET samples 


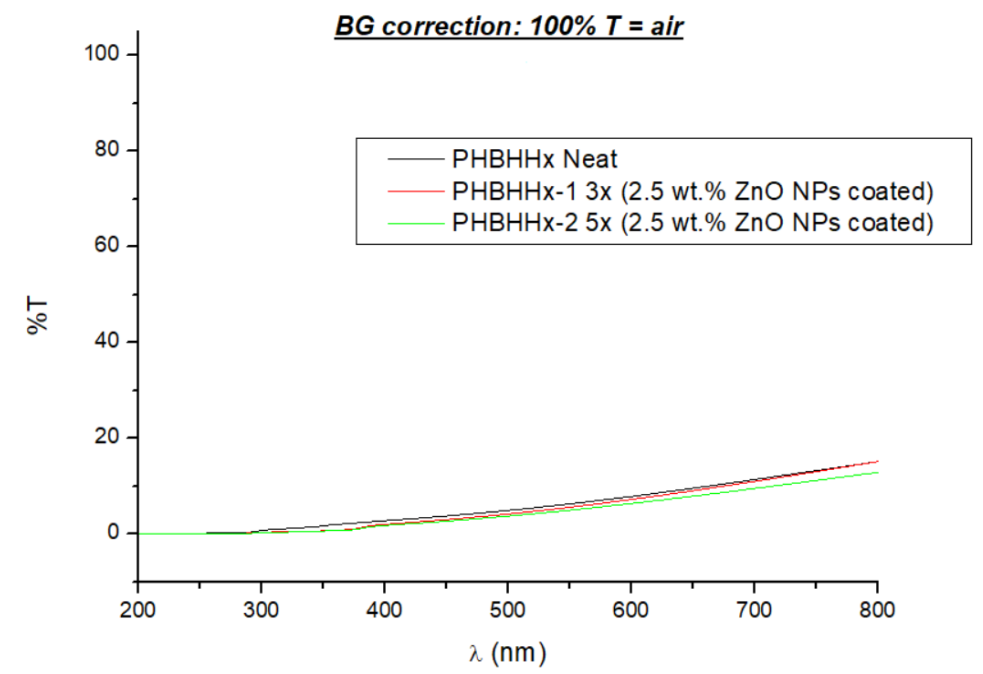

Graph 2: UV-vis spectra of neat, 3x and 5x ZnO NPs coated PHBHHx samples

\section{Conclusion}

Ultrasonic spray coating method was employed to deposit $\mathrm{ZnO}$ nanoparticles on PET and self-made PHBHHx substrates. $2.5 \mathrm{wt} . \% \mathrm{ZnO}$ NPs solution was used for spray coating these substrates under the optimized conditions of the spray coater. For 50x coated PET substrates, the OTR results showed a 5\% decrease in OTR value and no change in OTR value for 50x coated PHBHHx substrates - as compared to their neat substrates. Less tortuous path and hydrophobic behaviour caused this oxygen transmission trend in case of coated PET and PHBHHx substrates, respectively. For UV barrier properties, ZnO NPs showed their good UV barrier property for both substrates, even with 3 and 5 no. of coated layers. These results showed an economical way to block UV light by using $\mathrm{ZnO}$ NPs coated substrates.

\section{Acknowledgements}

The authors would like to express their sincere gratitude to the Higher Education Commission (HEC), Pakistan, under award letter No. PD (HRDI-UESTPs)/Batch-IV/2016/1450, for providing research grant for this work.

\section{References}

1. L. B. Modesto-López, M. Miettinen, T. Torvela, A. Lähde, and J. Jokiniemi, "Direct deposition of graphene nanomaterial films on polymer-coated glass by ultrasonic spraying," Thin Solid Films, vol. 578, pp. 45-52, 2015/03/02/ 2015.

2. Spray shaping systems - AccuMist ${ }^{\mathrm{TM}}$ ultrasonic spray shaping, Available: https://www.sonotek.com/ultrasonic-coating/spray-shaping-systems/, accessed on 19 July, 2019.

3. J. Stryckers, L. D'Olieslaeger, J. V. M. Silvano, C. K. Apolinario, A. C. G. Laranjeiro, J. Gruber, et al., "Layer formation and morphology of ultrasonic spray coated polystyrene nanoparticle layers," physica status solidi (a), vol. 213, pp. 1441-1446, 2016.

4. What is the $\mathrm{ZnO}$ nanoparticles $\mathrm{UV}$ spectrum range? Available: https://www.researchgate.net/post/What_is_the_ZnO_nano_particles_UV_spectrum_range, accessed on 19 July, 2019. 


\title{
Water pumping through microchannels
}

\author{
Z. Ramazanova ${ }^{1}$, Z. Insepov $^{1-3 *}$ \\ ${ }^{1}$ Nazarbayev University, 53 Kabanbay Batyr Ave, Nur-Sultan, Kazakhstan, zamart.ramazanova@nu.edu.kz \\ ${ }^{2}$ Purdue University, 500 Central Dr., West Lafayette, IN 47907, USA, zinsepov@purdue.edu \\ ${ }^{3}$ National Research Nuclear University (MEPhI) 31 Kashira Hwy, Moscow Russia
}

\begin{abstract}
Finite element modelling results are presented on water actuation via a sub-millimeter thin tube by acoustic waves. Basic principles of water actuation techniques for water transport in micron scale tubes are discussed. The tube walls have exposed the frequency of acoustic waves of $37 \mathrm{kHz}$ that showed a substantial increase in the flow rate of water and fountaining at a resonant frequency. Experimental observations of acoustic streaming and pumping effects via sub-millimeter tubes were confirmed by numerical finite-element simulations of water flows under acoustic waves. It was observed that for the same values of the immersion depths of the tubes, the water level increased with the decreasing of the tube diameter. For the full range of diameters, there was a local maximum of water level at the immersion depth. As input data in the simulation model, the following parameters were specified: a frequency of the wave, sound speed in water, the radius of the tube, and the density of the liquid. The flow rate and the mean flow velocities in the inlet and outlet openings were evaluated in the calculations and compared with experiment.
\end{abstract}

Keywords: surface acoustic wave, acoustic streaming, water droplet, finite element modelling, liquid pumping

\section{Introduction}

A significant engineering problem exists in the development of a liquid actuation technology that enables high flow fluxes with lower parasitic power losses in micro fuel cell systems. Current fabrication processes are incapable of providing high volumetric liquid fluxes at affordable prices [1]. Today motor-driven air delivery uses about $15 \%$ to $20 \%$ of the system power available from micro fuel cells. In addition, the costs of such pumps are about ten times higher than the projected market will likely accept [2-4].

Therefore, a novel nano- and micro- pumps are desirable that will be capable of pumping gases and liquids at the nanoscale, through channels with diameters as small as $1-10 \mathrm{~nm}$ and $1-100 \mu \mathrm{m}[2,3,4]$. Such pumps will be important devices in cell biology for facilitation, acceleration, and control of the ion exchange into and out of a cell; in medicine, for selective drag delivery in a human body; in energy technology, for hydrogen storage in carbon nanotubes and for hydrogen transport through them.

In this work, we present our experiment on water pumping via micron-scale tubes driven by acoustic waves (Fig. 1) and finite element method modelling (Fig. 2) of this process.
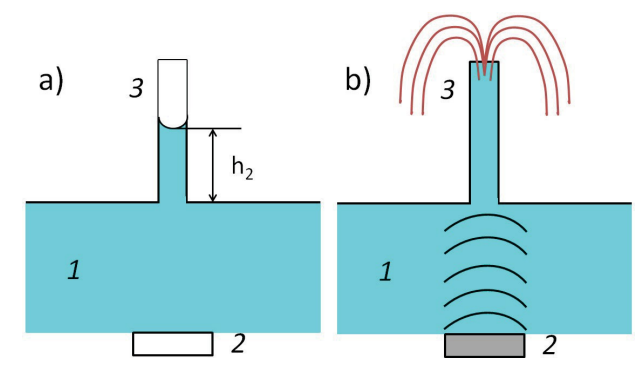

Figure 1 - a) Schematics of experimental set-up for water (1) pumping via a micron scale tube (3); b) Fountain of water through the tube after an ultrasonic transducer (2) was turned on

\footnotetext{
* Corresponding author: zinsepov@pudue.edu
}

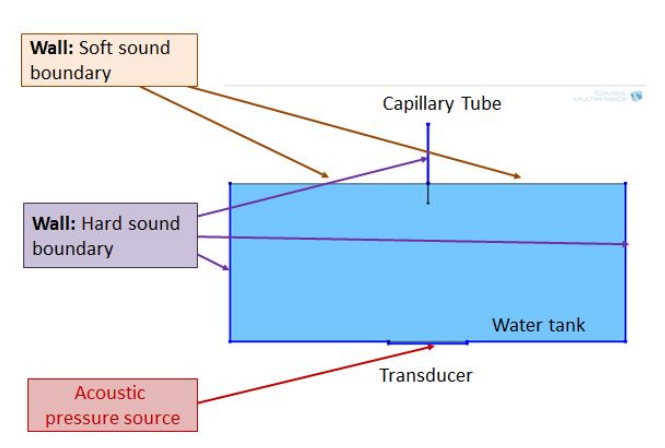

Figure 2 - Schematic illustration of geometry object 


\section{Experiment}

The present work studies the effect of surface acoustic waves with frequency of $37 \mathrm{kHz}$ on the flow rate of water through a tube with a diameter of 0.1-0.6 mm. Fig. 1a shows the experimental setup. We studied the influence of ultrasound on flow actuation in a tube and the schematic of fountaining is shown in Fig. 1b. The experiment fragments are shown in Fig. 3.

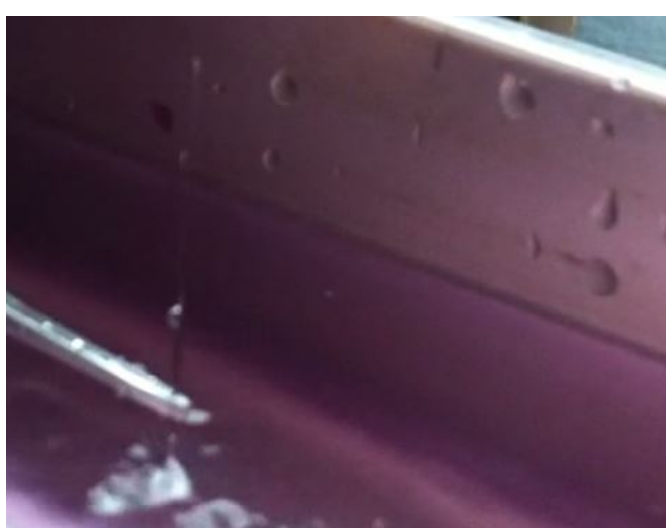

Figure 3 - Fragments the fountaining of water through a sub-millimeter thin tube

The experiment performed at various values of tube diameter, $\mathrm{d}$ (with the diameters ranging between 0.3-0.6 $\mathrm{mm}$ ), and the immersion depth of the capillary tip, $\mathrm{y}$, to establish the dependence of the rising the fountain height $\mathrm{h}$ on diameter and the depth. The results illustrated in Fig.4 indicate the followings:

1) At the same value of the immersion depth, the water level increases with the decreasing of the tube diameter.

2) For full range of diameter, there is a local maximum of water level at the immersion depth of 9-13 mm.

The experimental results showed a significant increase in the water flow through the tube. After it was placed in water and an ultrasound acoustic transducer at the bottom of the vessel started to generate an ultrasound signal. Water started to fountain from the open end of the capillary [5].

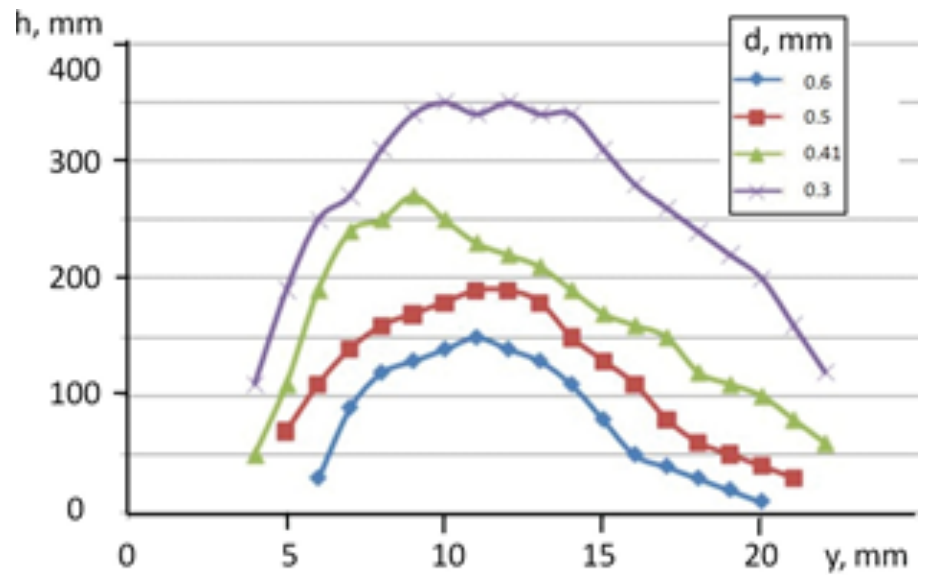

Figure 4 - Dependence of the water fountain height $h$ flow via the tube on the depth of tube tip immersion into water and on the tube diameter $d .(d=0.3-0.6 \mathrm{~mm})$

\section{Simulation}

We investigate numerically actuation of water flow in a sub-millimeter tube and the effect of ultrasound on water droplet using the finite element method (FEM) by COMSOL Multiphysics software [6]. COMSOL Multiphysics package is a powerful interactive environment for the modeling and calculation of most scientific and engineering problems based on the partial differential equations (PDE) with the method of finite elements. The software runs the finite element 
analysis together with the grid taking into account the geometric configuration of the body and control errors by using a variety of numerical solvers. It is a computer program that allows us to model and simulate a wide variety of physical phenomena. The program can tackle technical problems in many fields of science and engineering. Here are some of them: acoustics, electromagnetism, fluid dynamics and heat transfer, structural mechanics, chemical engineering, earth science etc. The fact that COMSOL Multiphysics is capable of analyzing our problems and that it is relatively user -friendly made it a better choice. The wide usage of COMSOL Multiphysics in various domains largely depends on its marked characteristics. These characteristics are: i) It can be used to solve multi-physics problems; ii) the user can specify his order own Partial Differential Equations; iii) Professional predefined modeling interfaces; iv) CAD models can be made directly; v) CAD package can be added.

The Navier-Stokes and acoustic pressure equations were solved simultaneously to determine the hydrodynamic field in the tube. Our method of solving the hydrodynamic equations for sound propagation in water captures all wave effects, including diffraction. Figure 5 shows 2D axisymmetric system geometry of simulation which shows a tank and a capillary where the blue area indicates water, and the red area - air. In our case, alternating pressure on the inlet will promote oscillating water inflow within capillary and filling of upper reservoir with water. This can be observed on the snapshots illustrated in Fig. 5.

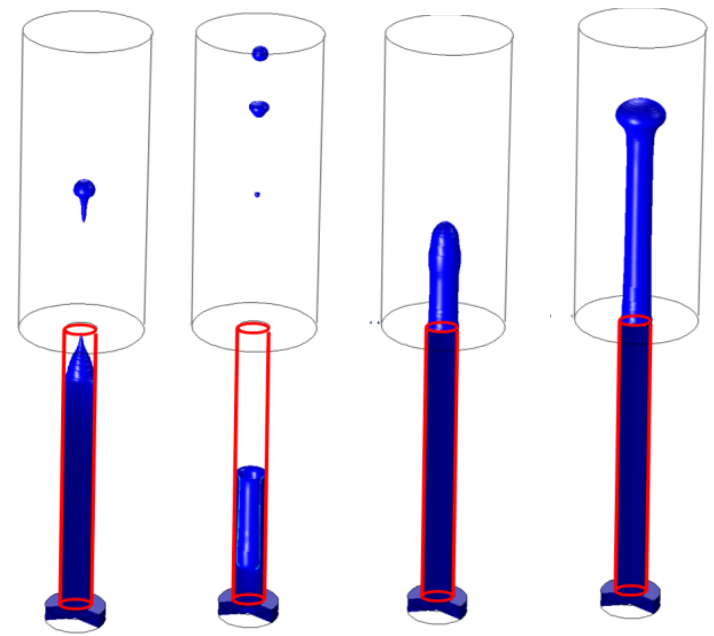

Figure 5 - Image sequences of oscillating water inflow within capillary

The Laminar Two-Phase Flow, Phase Field (tpf) was used for our simulation, providing the velocity field, the pressure, and the phase- field variables [6]. Gravity force was taken into account. A $3 \mathrm{~mm}$ length tube, with various diameters $(\mathrm{d}=0.3 \div 0.6 \mathrm{~mm})$ has a wetted wall with a contact angle of $70^{\circ}$. Effects of gravity field, of ambient pressure were also included into our simulation. The diameter of the capillary tube was $0.3 \mathrm{~mm}$. The total power of the transducer was $150 \mathrm{~W}$. The frequency of the emitted ultrasound was $37 \mathrm{kHz}$. The driving force of water propagation in the capillary was periodically alternating pressure governed by equation (1) and (2) applied on the inlet with pressure, no viscous stress boundary condition:

$$
\begin{gathered}
P=A \sin (k x-\omega t \\
P=P_{0}\left[\mu\left(\nabla \mathrm{u}+(\nabla \mathrm{u})^{T}\right)\right] \mathrm{n}
\end{gathered}
$$

where $P$ - pressure, $A$ - amplitude, $k$ - wavenumber, $x$ - position, $\omega$ - angular frequency, $t$ - time, $n$ - boundary normal pointing out of the domain, $u$ - velocity magnitude, $\mu$ - dynamic viscosity, and $T$ - the total stress tensor.

Water flow at the boundary of the inlet was governed by the Navier-Stokes equation (3):

$$
\rho \frac{\partial u}{\partial t}+\rho(u \cdot \nabla) u=\nabla \cdot\left[-p I+\mu\left(\nabla \mathrm{u}+\nabla \mathrm{u}^{T}\right)\right]+\mathrm{F}_{g}+F_{s t}+F_{e x t}+F
$$

where $\rho$ - density, I - identity matrix, T - total stress tensor, $\mathrm{F}$ - body forces.

Also, we have modelled the process which is shown in Fig. 1 experimental set-up for water pumping via a micron-scale tube with another geometry configuration to calculate the acoustic pressure and velocity 
of the water. Figure 2 shows the modelled geometry object. We used for the modelling an Acoustic pressure transient model in COMSOL Multiphysics with soft sound and hard sound boundary conditions for a water tank.

Figure $6(\mathrm{a}, \mathrm{b})$ shows our numerical results regarding pressure distribution and its alternation in the capillary system. It can be seen that pressure alternates periodically, and this kind of pressure alternations allow us to simulate ultrasound acoustic radiation pressure impact on the subsurface of water.

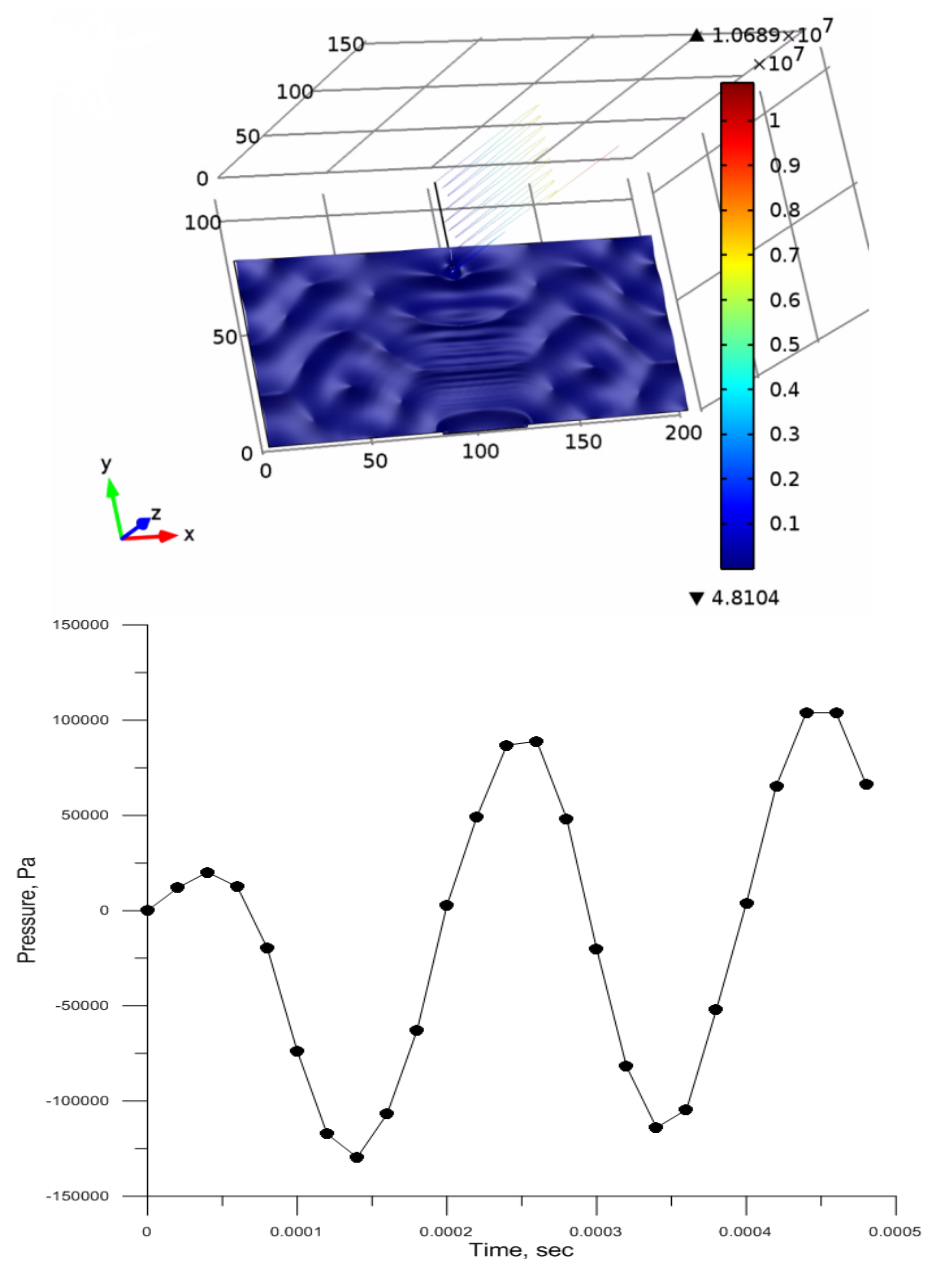

Figure 6 - a) Acoustic pressure distribution in the capillary system; b) Plot of alternating pressure

According to simulation water flow velocity at maximum is $8 \mathrm{~m} / \mathrm{s}$ (higher velocity on graphs corresponds to air flow). In addition, flow velocity alternates as well due to pressure alternation. Variation of velocity magnitude of flow due to interaction with acoustic pressure is shown in Fig. 7. 


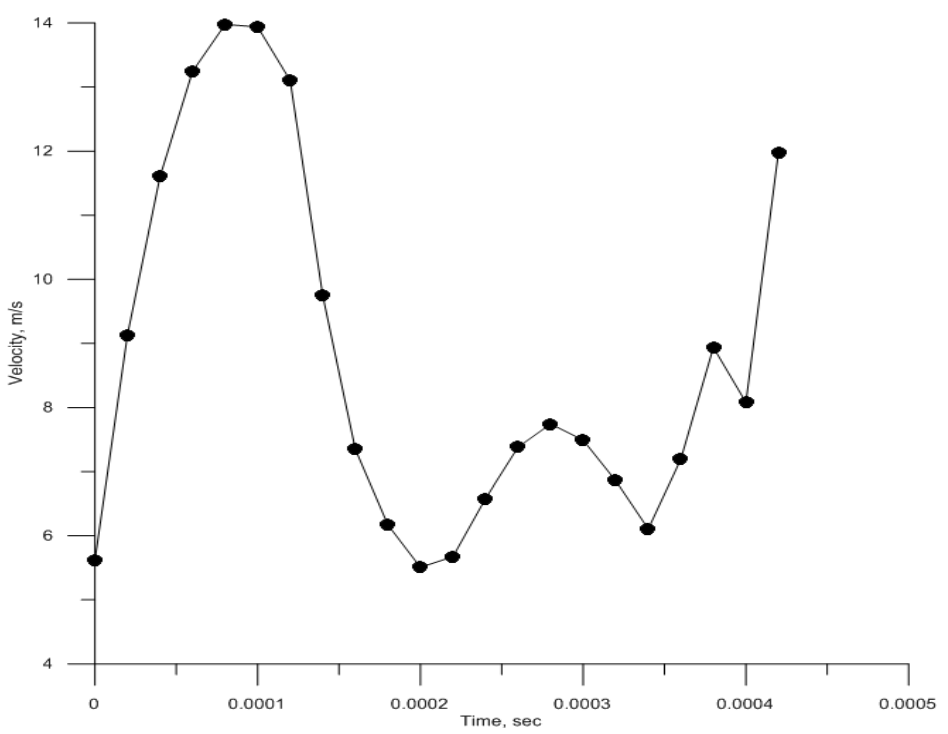

Figure 7 - Flow velocity variation in the capillary system

The explanation of this phenomenon is the oscillation of fluid inside the tube under the influence of ultrasound source, which causes the sharp growth of capillary effect, and level of water in capillary increases several dozen times. It was experimentally proved that the flow is activated not because of the radiation pressure or capillary forces, but because of the standing ultrasound waves.

\section{Summary}

Experimental and numerical studies of the water actuation via a sub-millimeter thin tube by acoustic waves have been conducted to investigate the effects of parameters such as frequency, acoustic pressure, flow rate and velocity magnitude. In the experiment showed a substantial increase in the flow rate of water and fountaining at a resonant frequency which confirmed by numerically of water flows under acoustic waves. The propagation of ultrasound waves in fluids generates the fluid flow, so-called acoustic streaming, the velocity of which depends on viscosity of the media, sound intensity and on sound frequency. In general, the magnitude of this stream is low enough and about a percent of ultrasound velocity.

\section{Acknowledgements}

This work was supported in part by the Nazarbayev University World Science Stars program, under Grant \# 031-2013 of 12/3/2013. The authors would like to thank Ardak Ainabayev, Galiulla Imanbayev and Bakhytnur Berdenova for contribution the manuscript.

\section{References}

1. RNCOS report, "Fuel Cell Industry Analysis", June 2011.

2. Z. Insepov, D. Wolf, A. Hassanein, Nano Letters 6 (2006)

3. Z. Insepov, R. Miller, J. Nanotechnology in Eng. and Medicine, 3 (2012) 025201-1

4. Z. Insepov, A. Hassanein, US Patent \#7,632,482 (Dec. 15, 2009); US Patent \# 7,651,673 (Jan. 26, 2010 )

5. B.Berdenova, A. Ainabayev, Z. Insepov et al., NSTI-Nanotech 2014, ISBN 978-1-4822-5827-1 Vol.2, 2014

6. Www.comsol.com 


\title{
Fabrication of MEMS based blackbody for calibration of infrared camera using black silicon and carbon nanotubes
}

\author{
Seolhui Hwang ${ }^{1}$, Jae Hyuk Lee ${ }^{2}$, Taegyu Kim ${ }^{3}$, Jong G. Ok ${ }^{2}$, Jongkwang Lee ${ }^{1}$ \\ ${ }^{1}$ Hanbat National University \\ 125, Dongseo-daero, Yuseong-gu, Daejeon, Republic of Korea, tsb03182@naver.com \\ ${ }^{2}$ Seoul National University of Science and Technology \\ 232, Gongneung-ro, Nowon-gu, Seoul, Republic of Korea, jaehyuk1015@naver.com \\ ${ }^{3}$ Chosun University \\ 309, Plimun-daero, Dong-gu, Gwangju, Republic of Korea, taegyu@Chosun.ac.kr \\ ${ }^{2}$ Seoul National University of Science and Technology \\ 232, Gongneung-ro, Nowon-gu, Seoul, Republic of Korea, jgok@seoultech.ac.kr \\ ${ }^{1}$ Hanbat National University \\ 125, Dongseo-daero, Yuseong-gu, Daejeon, Republic of Korea, jongkwang@hanbat.ac.kr
}

\begin{abstract}
Optical cameras are not uniform in output characteristics due to high temperature low temperature repetition and repetitive operation non-operation. This is a fatal disadvantage which deteriorates the performance of the optical camera, and a blackbody is used to solve this problem. Black silicon (BSi) and multi-walled carbon nanotubes (MWCNTs) were proposed for fabricating of MEMS based blackbody surface on upper side of a silicon wafer of this system. To fabricate the $\mathrm{BSi}$, we used the cryogenic and Bosch processes in the DRIE process. The detailed experimental variables of the cryogenic process are $: \mathrm{SF}_{6} / \mathrm{O}_{2}$ gas $=40 / 18 \mathrm{sccm}, \mathrm{RF}$ power : $1000 \mathrm{~W}$, pressure : $10 \mathrm{mTorr}$, process time : 15 minutes, process temperature : $-110{ }^{\circ} \mathrm{C}$. Also, the detailed experimental variables of the Bosch process are : $\mathrm{SF}_{6} / \mathrm{C}_{4} \mathrm{~F}_{8}$ gas $=200 / 200 \mathrm{sccm}$, RF power : 2000 $\mathrm{W}, \mathrm{SF}_{6} / \mathrm{C}_{4} \mathrm{~F}_{8}$ pressure : $30 / 40 \mathrm{mTorr}, \mathrm{SF}_{6} / \mathrm{C}_{4} \mathrm{~F}_{8}$ pulse time $=3 / 0.5$ seconds, bottom power : $40 \mathrm{~W}$. The total process time is 5 minutes. MWCNTs were cultivated using a chemical vapored deposition (CVD) process. In order to cultivate MWCNTs, a catalyst layer was required, and Aluminum oxide $\left(\mathrm{Al}_{2} \mathrm{O}_{3}\right) 10 \mathrm{~nm}$ and iron $(\mathrm{Fe}) 1 \mathrm{~nm}$ were used as catalysts. Then, the substrate was placed in a furnace and Ar was supplied at $400 \mathrm{sccm}$, $\mathrm{H}_{2}$ at $200 \mathrm{sccm}$, and $\mathrm{C}_{2} \mathrm{H}_{4}$ at $100 \mathrm{sccm}$ for $10 \sim 15$ minutes at a temperature range $725^{\circ} \mathrm{C} \sim 775^{\circ} \mathrm{C}$. MWCNTs cultivated on the surface of BSi can be seen from the results of SEM measurements and show that nano- or micro-sized MWCNTs are well grown on the BSi surface. As a result, it was found that the high reflectance $(20 \sim 50 \%)$ of BSi in the infrared range $(6 \sim 14 \mu \mathrm{m})$ was greatly reduced to $2 \sim 5 \%$ or less in MWCNTs growth.
\end{abstract}

Keywords: Deep reactive ion etching (DRIE), Black silicon (BSi), Chemical vapor deposition (CVD), Multiwalled carbon nanotubes (MWCNTs)

\section{Introduction}

Currently, the information provided by satellites plays a role in forecasting weather and observing the earth. Also, as the information age becomes more and more, the role of satellites becomes more important. These satellites are composed of various elements, among which an optical camera is mounted for carrying out earth observation missions.

The optical camera acts as the eye of a satellite, detects the light reflected from the earth, and creates images to collect image information. At present, optical cameras are not uniform in output characteristics due to the repetition of high temperature-low temperature and repeated operation - non-operation [1]. This is a major factor that deteriorates the performance of the optical camera. Therefore, in order to receive high-quality images through an optical camera, it is necessary to correct these problems.

A blackbody system is used to correct the non-uniform output characteristics of current optical cameras. Blackbody systems have been steadily progressing to improve the performance of optical cameras. However, the existing blackbody system requires a very large and complicated device, which makes it difficult to obtain a uniform temperature of the blackbody surface.

Therefore, this study proposed a blackbody system using MEMS technology. As shown in figure 1, the black body consists of a silicon wafer, which increases the thermal conductivity. In addition, the back side of the 
silicon wafer integrated the microheater and the temperature sensor to increase the sensitivity of the sensor to improve the response characteristics, and the front surface of the silicon wafer realized the low reflectance on the black body surface. This unified system has advantages in that it can be simplified compared to the existing black body system and no complicated device is required.

The overall mechanism of this system and the development of microheaters and micro-heaters and temperature sensors on the back of silicon wafers have been completed. At present, it is necessary to fabricate a low reflectance blackbody surface on the front side of a silicon wafer. The low-reflectance blackbody surface fabricated using MEMS technology utilizes shape and material properties. Therefore, we will utilize multiwalled carbon nanotubes (MWCNTs) with black silicon and (BSi) material properties using geometrical properties to fabricate black body surfaces based on MEMS technology. To do this, MWCNTs are grown on the surface of BSi after BSi is fabricated.

$\mathrm{BSi}$ is an effective material for reducing light in the visible light range by forming various shapes of nanomicro size on 8- inch silicon wafer surface. It is fabricated through deep reactive ion etching (DRIE) and is manufactured without a mask, which simplifies the process and reduces the cost of the process.

MWCNTs are carbon isotopes having a cylindrical nanostructure and are used in various fields due to their low reflectance and high thermal conductivity. It was fabricated on the BSi surface to maximize the light reduction effect in the infrared region. It was fabricated by chemical vapor deposition (CVD).

In this study, the performance of the black body system was evaluated by measuring the reflectance of the manufactured BSi and MWCNTs. Fourier transform infrared (FT-IR) was used for the reflectance measurement and scanning electron microscope (SEM) was used for the surface analysis. Figure 2 shows a schematic view of the MWCNTs grown on the BSi surface.

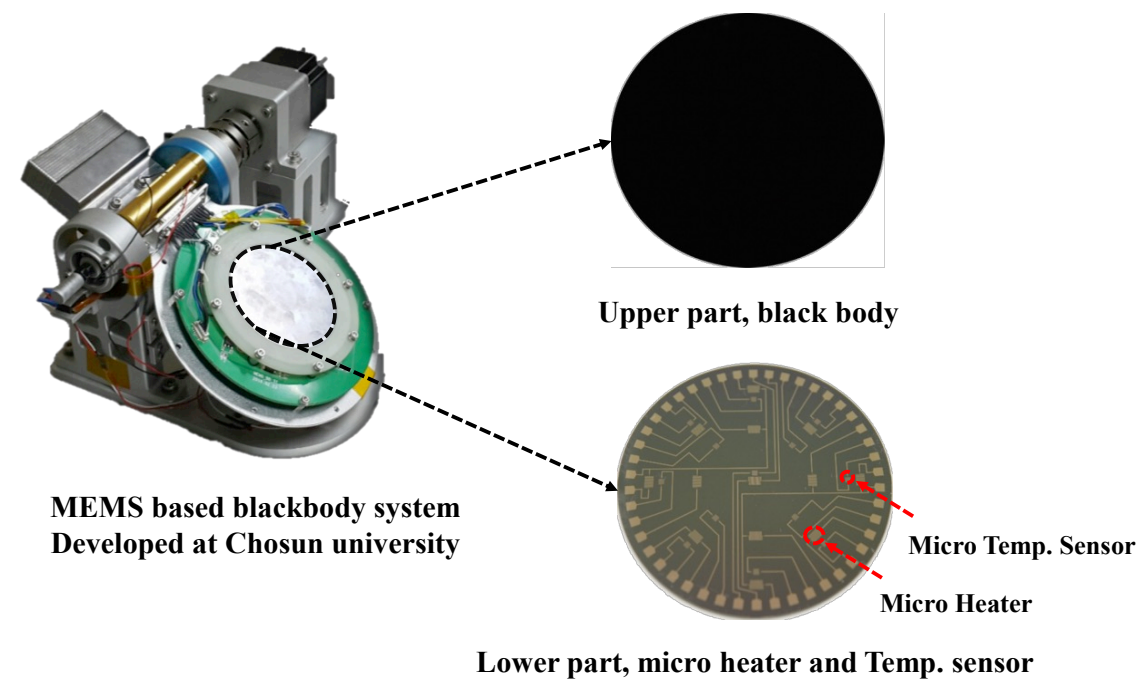

Fig. 1: The entire system of a blackbody based on MEMS.

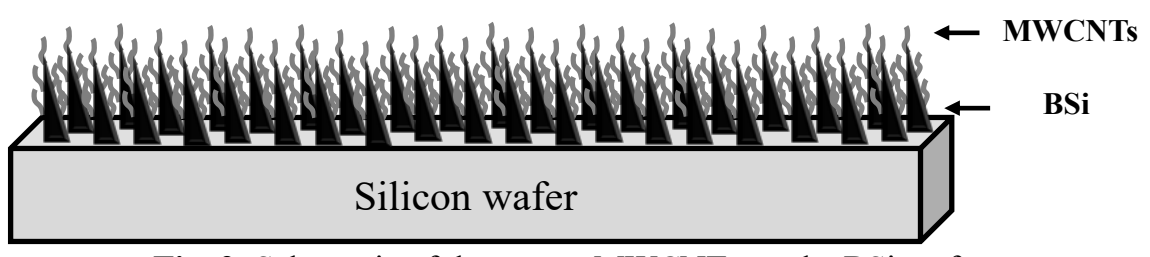

Fig. 2: Schematic of the grown MWCNTs on the BSi surface.

\section{Experiment}

\subsection{Fabrication method of black silicon}

DRIE is an anisotropic process for manufacturing high aspect ratio holes and trenches using cryogenic and Bosch processes. Generally, during the DRIE process, only the desired part is processed using the mask, but the surface is formed without using the mask when manufacturing the BSi. In this experiment, we tried to confirm the change of BSi shape due to the process change using both the cryogenic process and the Bosch process. The BSi fabrication process through the DRIE process is performed alternately with the passivation 
step and the etching step. As shown in figure 3, the etch step occurs for a certain period of times, and then the process of generating the passivation step is repeated to change the surface roughly, and then the BSi is fabricated [2].

Octafluorocyclobutane $\left(\mathrm{C}_{4} \mathrm{~F}_{8}\right)$ is commonly used gas in the passivation step of the Bosch process, and $\mathrm{O}_{2}$ is commonly used gas in the passivation step of the cryogenic process. In addition, sulfur hexafluoride $\left(\mathrm{SF}_{6}\right)$ is mainly used gas in etching step [2]. The DRIE process is influenced by many factors such as formation temperature, chamber cleanliness, and process temperature, among which process parameters are most affected [3]. Process parameters include gas flow rate, process time, and chamber pressure [4].

\subsection{Cryogenic process}

Experimental details of cryogenic process are $: \mathrm{SF}_{6} / \mathrm{O}_{2}$ gas $=40 / 18$ [sccm], RF power $=1000$ [W], chamber pressure $=10[\mathrm{mTorr}]$, total process time $=15[\mathrm{~min}]$, process temperature $=-110\left[{ }^{\circ} \mathrm{C}\right]$.

\subsection{Bosch process}

Experimental details of cryogenic process are $: \mathrm{SF}_{6} / \mathrm{C}_{4} \mathrm{~F}_{8}$ gas $=200 / 200$ [sccm], $\mathrm{RF}$ power $=2000$ [W], $\mathrm{SF}_{6}$ $/ \mathrm{C}_{4} \mathrm{~F}_{8}$ chamber pressure $=30 / 40[\mathrm{mTorr}], \mathrm{SF}_{6} / \mathrm{C}_{4} \mathrm{~F}_{8}$ pulse time $=3 / 0.5$ [sec], bottom power $=40$ [W], total process time $=5[\mathrm{~min}]$.

\subsection{Fabrication method of MWCNTs}

CVD is a process in which a substrate on which a catalyst is deposited is placed in a reactor and various gases are injected to grow CNTs. This is a process in which after the heat treatment is performed in an $\mathrm{H}_{2}$ atmosphere at a high temperature of 700 to $950^{\circ} \mathrm{C}$, a hydrocarbon is supplied into the reactor, and the residual hydrocarbon gas is exhausted with Ar gas. Through this process, MWCNTs are grown. In this study, the CVD process was performed using a Labview control-based furnace manufactured by Seoul National University of Science and Technology.

The catalyst layer was deposited to grow MWCNTs on the BSi surface. $10 \mathrm{~nm}$ of aluminum oxide $\left(\mathrm{Al}_{2} \mathrm{O}_{3}\right)$ and $1 \mathrm{~nm}$ of iron $(\mathrm{Fe})$ were used as catalyst materials. Since MWCNTs are grown by catalyst particles, catalyst layer deposition is very crucial [6]. Figure 4 shows a schematic diagram of the order in which MWCNTs are grown on the BSi surface.

The detailed process parameters used here provide argon $(\mathrm{Ar})$, hydrogen $\left(\mathrm{H}_{2}\right)$, and ethane $\left(\mathrm{C}_{2} \mathrm{H}_{4}\right)$ gases in the temperature range of $725^{\circ} \mathrm{C}$ to $775^{\circ} \mathrm{C}$. The flow rate of Ar was $400 \mathrm{sccm}$, the flow rate of $\mathrm{H}_{2}$ was $200 \mathrm{sccm}$, and the flow rate of $\mathrm{C}_{2} \mathrm{H}_{4}$ was $200 \mathrm{sccm}$. The process time of each gas was 10 to 15 minutes.

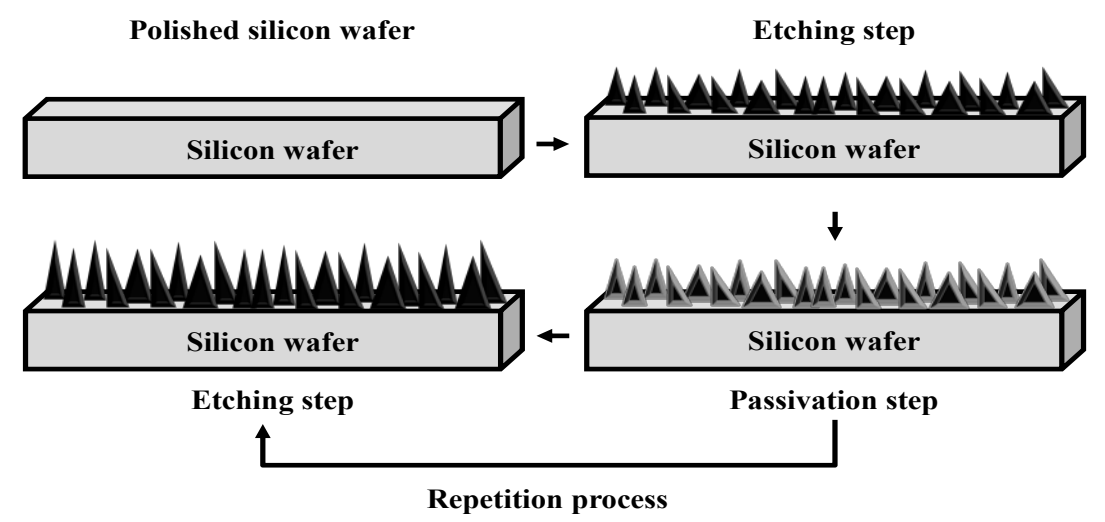

\section{Results and discussion}

Fig. 3: The progress of DRIE.

Figure 4 shows the surface where only BSi is present and the surface where MWCNTs are grown on BSi surface. Surface analysis was performed via SEM. Figure 4 shows that the BSi fabricated through the cryogenic process has a grass-like structure and the length is about $668 \mathrm{~nm}$. In addition, the BSi fabricated through the Bosch process has a needle structure and has a length of about $2.31 \mu \mathrm{m}$. These results show that MWCNTs of several hundred nanometers in length can grow better on fabricated BSi surfaces through cryogenic processes. 
Figure 5 shows the surface reflectance results for BSi only and MWCNTs grew on BSi surface. Reflectance measurements were carried out in the wavelength range of $6 \mu \mathrm{m}$ to $14 \mu \mathrm{m}$ through FT-IR to measure the light of the actual optical camera operating wavelength range.

As a result of the reflectance measurement, the reflectance of the BSi fabricated by the cryogenic process was about $20 \%$, and the reflectance of the BSi fabricated by the Bosch process was about $50 \%$. This result indicates that BSi can't be used as a black body at the wavelength range of the optical camera. However, when MWCNTs grow on the surface of BSi, the reflectance decreases to about $2 \sim 5 \%$. The results show that the MWCNTs applied to the surface of BSi significantly reduce the reflectance in the infrared range and can be used as a blackbody system.
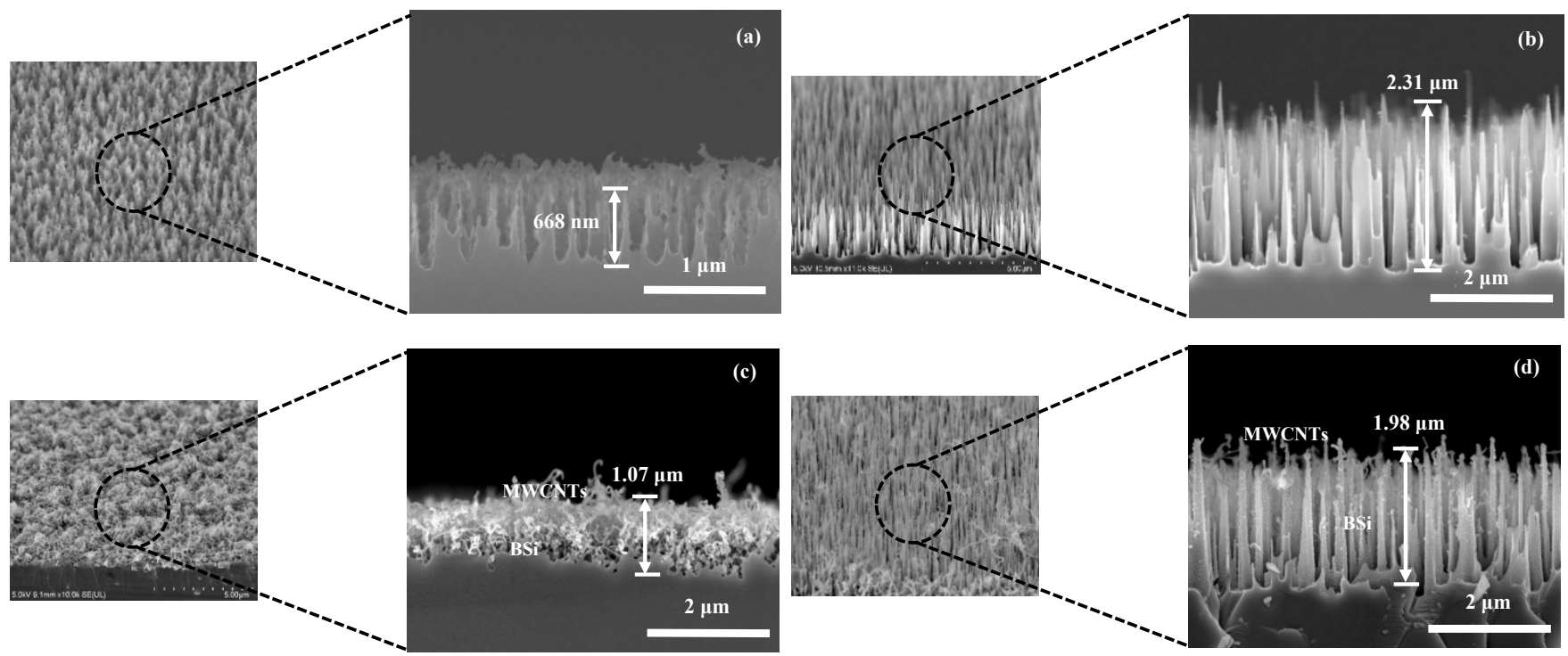

Fig. 4: The comparison of morphology the BSi and MWCNTs grown on BSi. (a) SEM image of BSi fabricated by cryogenic process. (b) SEM image of BSi fabricated by Bosch process. (c) SEM image of MWCNTs grown on BSi fabricated by cryogenic process.

(d) SEM image of MWCNTs grown on BSi fabricated by Bosch process.
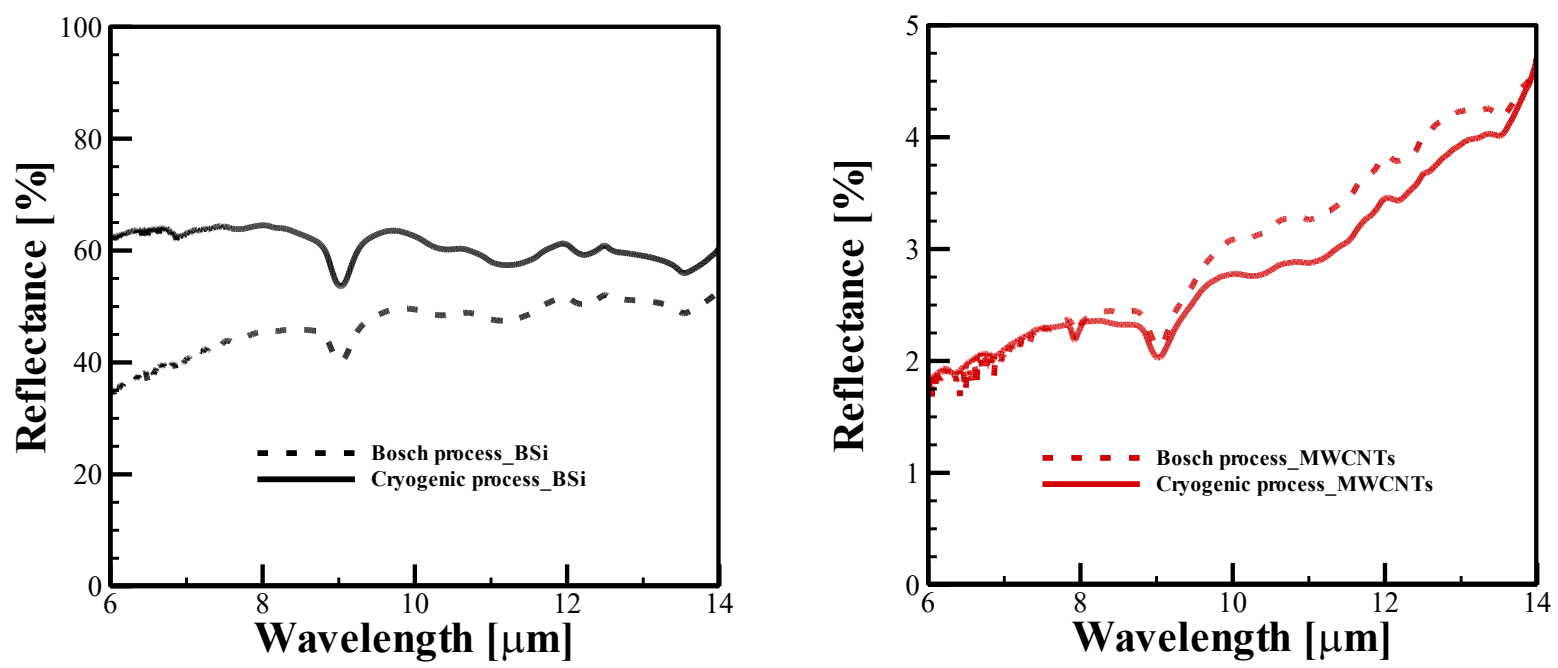

Fig. 5: The reflectance of BSi (left) and MWCNTs grown on BSi (right) measured by FT-IR.

\section{Conclusion}

In this study, we proposed a MEMS-based blackbody surface to fabricate a blackbody system for calibration of satellite infrared cameras. In order to fabricate the MEMS-based black body surface, we used BSi using shape features and MWCNTs using material properties. The DRIE process was used to fabricate the BSi, and the BSi fabricated through the cryogenic process had a grass-like shape of about $668 \mathrm{~nm}$ in size. In addition, the BSi fabricated through the Bosch process has a needle shape of about $2.32 \mu \mathrm{m}$ in size. The MWCNTs were 
grown using a CVD process, and growth was observed on the BSi surface with a grass-like shape. To evaluate the performance of the surface of a black body based on MEMS, the reflectance was measured by FT-IR. As a result of the reflectance measurement, BSi shows a reflectance as high as about 40 to $60 \%$, but when the MWCNTs are grown, the reflectance greatly decreases by about 2 to $5 \%$. These results show that the MWCNTs grown on the BSi surface can have a performance that can be utilized as a black body.

\section{Acknowledgements}

This research was supported by Basic Science Research Program through the National Research Foundation of Korea (NRF) funded by the Ministry of Education (NRF-2016R1D1A1B03934309).

\section{References}

1. Jin. K.W, Joo. G.H and Yang. K.H, "Research Trend of Calibration Methods on the Satellite Visible and Infrared Sensor", Current Industrical and Technological Trends in Aerospace, Vol. 7. No. 1, pp. 89-96, 2009.

2. Steglich. M, Käsebier. T, Zilk. M, Pertsch, T. Kley, E-B. and Tünnermann, A, "The structural and optical properties of black silicon by inductively coupled plasma reactive ion etching," J. Applied Physics, Vol. 116. 173503. pp. 1-12, 2014.

3. Tian-Le Gao, Xiao-Sheng Zhang, Guang-Yi Sun and Hai-Xia Zhang, "Fabrication of Superhydrophobic Wide-band "Black Silicon" by Deep Reactive Ion Etching," Proceedings of the IEEE International Conference on Nano/Micro Engineered and Molecular Systems, Kaohsiung, Taiwan, pp. 209-212, 2011.

4. Wasilik, M and Chen, Ning, "Deep Reactive Ion Etch Conditioning Recipe," Proceedings of the SPIE, Vol. 5342, pp. 103-110, 2004. 


\title{
Carbon dots photoluminescence technique to detect total Chromium in industrial wastewater
}

\author{
Rupam Sinha ${ }^{\mathrm{a}}$, Ravula Rajasekhar ${ }^{\mathrm{b}}$, and Tapas K Mandal ${ }^{\mathrm{a}, \mathrm{b}, \mathrm{c}^{*}}$ \\ ${ }^{a}$ Department of Chemical Engineering, Indian Institute of Technology Guwahati, Assam, \\ 781039, India \\ ${ }^{\mathrm{b}} \mathrm{Centre}$ for the Environment, Indian Institute of Technology Guwahati, Assam, 781039, India \\ ${ }^{\mathrm{c}}$ Centre for Nanotechnology, Indian Institute of Technology Guwahati, Assam, 781039, India \\ *Email corresponding authors: tapasche@iitg.ac.in
}

\begin{abstract}
:
This current work focuses on the synthesis of photoluminescent (PL) CDs from indigenous potato sources by simple heating reaction. Functional groups of the synthesized CDs were characterized by FTIR spectrum and it was found that $-\mathrm{COOH}$ functional groups were attached to their surface. These carboxylic acids are responsible for their extraordinary water-solubility. The synthesized CDs exhibited a quantum yield (QY) of $6.08 \%$. The photoluminescent property of the CDs was utilized to develop a sensing technique for detection of total Chromium in industrial wastewater. Principal of selectively higher quenching of $\mathrm{CDs}$ by $\mathrm{Cr}^{6+}$ ions was utilized to detect Chromium in industrial wastewater.
\end{abstract}

\section{Introduction:}

Carbon quantum dots (CDs) are the new sensation in the scientific world in recent times. They are the new members of the carbon family having a diameter in the range of 2 to $10 \mathrm{~nm}$. CDs generally consist of $\mathrm{sp}^{2}$ hybridized carbon with crystalline or amorphous core and an oxidized carbon surface with carboxyl group. ${ }^{1}$ The inception of carbon quantum dots is actually an accidental experimental result obtained by $\mathrm{Xu}$ et al. in $2004 .^{2}$ They discovered this newly appointed carbon member during the separation and purification of single walled carbon nanotube (SWCNT). After the discovery of CDs, much research have been carried out to identify the properties and different applications of CDs. One of the most interesting properties of CDs is their photoluminescence property and because of this particular property CDs are considered as very useful photoluminescent probes. Also, CDs have gained more attention because of their other noticeable characteristics, like low toxicity, water solubility, high chemical stability, easy functionalization ${ }^{3}$ and impressive bio compatibility. ${ }^{4}$ All these properties attract the researches to extent the application of CDs in the fields of photocatalysis, ${ }^{5}$ bio-imaging, ${ }^{6-8}$ light emitting devices $^{9}$ and sensing ${ }^{10-12}$ etc. These fascinating properties of CDs and their wide range of applications have influenced different researchers to explore various sources to synthesize CDs. Some of the reported sources for CDs synthesis are orange juice, ${ }^{1}$ garlic, ${ }^{13}$ grape juice, ${ }^{14}$ banana juice ${ }^{15}$ etc. These bio-resources have been employed as the precursor of CDs because of their abundant nature, non-toxicity and low cost. Bio-resources contain full of carbohydrates such as starch, polysaccharide and sugar. The elements like $\mathrm{O}$ and $\mathrm{H}$ present in the carbohydrate (apart from $\mathrm{C}$ element), help to synthesize $\mathrm{CDs}$ with $\mathrm{OH}$ and $\mathrm{COOH}$ functional groups. These functional groups are the real reason behind the extraordinary water-solubility of CDs. To synthesize CDs from different bio resources, different methods have also been employed. For example, electrochemical method, ${ }^{16}$ hydrothermal method ${ }^{11}$ microwave synthesis technique, ${ }^{17-19}$ simple heating technique ${ }^{20}$ etc. are some of the different $\mathrm{CD}$ synthesis techniques. Among these methods, the hydrothermal process or simple heating reactions for $\mathrm{CD}$ synthesis have gained more popularity because of their easy control over reaction and simplicity in sample preparation.

In this work, we have synthesized $C D$ by simple heating reaction using indigenous potato as the bioprecursor. We used the photoluminescence property of CDs to detect total chromium in tannery water sample. We further checked the feasibility of $\mathrm{Cr}^{6+}$ detection system using real water sample (Tannery water) collected from Membrane separation laboratory, Indian Institute of Technology Guwahati, India.

\section{Experimental Section:}




\subsection{Materials}

$\mathrm{K}_{2} \mathrm{Cr}_{2} \mathrm{O}_{7}$, ethanol and $\mathrm{H}_{2} \mathrm{O}_{2}$ were purchased from Merck, India. All the chemicals were used without further purification. The potatoes were purchased from local market of Amingaon Market, Guwahati, India and were washed with water for further usage. The tannery water was collected from Membrane separation laboratory, Indian Institute of Technology Guwahati, India.

\subsection{Synthesis of photoluminescent CDs}

The photoluminescent CDs were synthesized by simple heating technique as described in our other work. ${ }^{21}$ In a typical method, $50 \mathrm{~g}$ of washed potato was blended with $125 \mathrm{ml}$ of de-ionized water by a mixer grinder (Samsung, MG 2573) and the mixture was heated at $150{ }^{\circ} \mathrm{C}$ for 90 minutes with constant magnetic stirring. Then the potato pulp was removed by simple filtration and $20 \mathrm{ml}$ of the liquid mixture was mixed with $20 \mathrm{ml}$ ethanol. This mixture was heated at $200^{\circ} \mathrm{C}$ for 2.5 hours. The remaining material after heating was then assorted in $20 \mathrm{ml}$ of de-ionized water. Afterwards, the solution was again filtered to remove the larger particles and the brownish filtrate was then dissolved in $30 \mathrm{ml}$ of ethanol. This brown CD solution was then centrifuged for 10 minutes at $12000 \mathrm{rpm}$ to remove extra remaining larger particles.

2.3 Characterizations

UV-vis spectra of the synthesized CDs were obtained by a UV-vis spectrophotometer (PerkinElmer, Lambda 35). Photoluminescent spectra were obtained by a photoluminescent spectrometer (PerkinElmer, LS 45). The Fourier transform infra red (FTIR) spectrum was obtained by FTIR spectrophotometer (Shimadzu, IRAffinity-1) in the range of 500-4000 $\mathrm{cm}^{-1}$. The crystallinity of CD sample was examined by X-ray diffraction analysis (XRD) using X-ray diffractometer (Burker, D8 Advance) between $20^{\circ}$ and $80^{\circ} 2 \theta$ values.

\subsection{Quantum yield calculation}

Quantum yield (QY) of the thus prepared CDs were calculated by the method used by Goswami et $a l .{ }^{22}$ in their work. In a typical method, QY of CDs was determined relatively with a reference photoluminescent sample quinine sulphate. The quinine sulphate reference was prepared by dissolving it in $0.1 \mathrm{M} \mathrm{H}_{2} \mathrm{SO}_{4}$ solution. The following formula was used to calculate the QY of CDs. Both the reference material and sample material were excited at same wavelength $(345 \mathrm{~nm})$,

$$
Q Y_{S}=Q Y_{R} \times \frac{A_{S}}{A_{R}} \times \frac{A b s_{R}}{A b s_{S}} \times \frac{\eta_{S}^{2}}{\eta_{R}^{2}}
$$

Where, $Q Y_{R}$ and $Q Y_{S}$ denote the quantum yield of reference and sample resepective. $A_{S}$ and $A_{R}$ refer to the area under the emission peak of sample and reference respectively at an excitation wavelength of $345 \mathrm{~nm}$ at slit width of $2 \mathrm{~nm} . A b s_{R}$ and $A b s_{S}$ are the absorbance reading of reference and sample respectively. $\eta$ refers to the refractive index of the solvent $(1.33$ for both sample and reference). The quantum yield of reference $\left(Q Y_{R}\right)$ is $54 \%$.

2.8 Detection of total Chromium

In the present method, total Chromium is detected in the form of $\mathrm{Cr}^{6+}$ ions since Chromium can alter the photoluminescence characteristics of CDs in this from only. In a typical method, a stock solution CDs was prepared with a concentration of $0.25 \mathrm{mg} / \mathrm{ml}$. Then $40 \mu \mathrm{L}$ of a particular concentration of $\mathrm{Cr}^{6+}$ was added to $5 \mathrm{ml}$ of CD solution. The final concentration of $\mathrm{Cr}^{6+}$ was ranging from 0 to $100 \mu \mathrm{M}$. The photoluminescence spectra were recorded at an excitation wavelength of $345 \mathrm{~nm}$ after 20 minutes of reaction at room temperature.

2.9 Oxidization of Tannery water using UV light

We oxidized the collected tannery water sample to convert all the $\mathrm{Cr}^{3+}$ ions into $\mathrm{Cr}^{6+}$ ions by using a previously established method reported by Rodman et al. ${ }^{23}$ In a typical method, we diluted the tannery water to $1: 2$ ratio with DI water. Then we added $0.1 \mathrm{ml} \mathrm{H}_{2} \mathrm{O}_{2}$ in the diluted tannery water and we transferred the mixture in a quartz photo reactor system. Then the reactor 
system was placed in a dark place and exposed to a UV light source $(75 \mathrm{~W}$, Rrimin Reptile Tortoise UVA UVB 3.0 Heating Lamp, Amazon) for 2.5 hours.

\section{Results and Discussion:}

The optical properties of the CDs were analyzed by UV-vis spectroscopy and photoluminescence spectroscopy. Figure 1 shows the UV-vis spectra and photoluminescence spectra of the aqueous solution of CD. Figure 1a demonstrates a continuous increase in absorbance value from $540-230 \mathrm{~nm}$ and a peak at $275 \mathrm{~nm}$ are observed. This peak refers to the $\pi-\pi^{*}$ and $n-\pi^{*}$ transition of electrons of the conjugated $\mathrm{C}=\mathrm{C}$ and $\mathrm{C}=\mathrm{O}$ bonds. Moreover along with this peak a long tail till $540 \mathrm{~nm}$ can also be observed which suggests the occurrence of CDs according to the previously reported articles. ${ }^{24-26}$ Figure $1 \mathrm{~b}$ shows the photoluminescence property of the $\mathrm{CD}$. The figure illustrates the dependency of photoluminescence intensities of the $\mathrm{CD}$ solution on different excitation wavelength ranging 305-405 nm. Initially, photoluminescence intensity rises with increasing excitation wavelength from $305-345 \mathrm{~nm}$, while the photoluminescence intensity starts decreasing along with a further increase in the excitation wavelength i.e $365 \mathrm{~nm}$ and onwards confirming that the synthesized CDs are mainly UV sensitive. The photoluminescence property of the CDs was quantified by calculating its Quantum Yield (QY) and the calculated QY value of the synthesized CDs is $6.08 \%$. The crystallinity of CD sample was also checked using XRD analysis and it is shown in figure 1c. No sharp peak is observed in this figure suggesting the amorphous nature of the synthesized CDs. Along with the XRD characterization, the characterization of surface functional groups of CDs was also done. For this, FTIR analysis was performed and it is shown in Figure 1d. It is observed from this figure, that there are two broad bands at around $3434 \mathrm{~cm}^{-1}$ and at $2940 \mathrm{~cm}^{-1}$. These two broad bands correspond to the stretching vibration of $-\mathrm{OH}$ (due to the presence of $-\mathrm{COOH}$ group) and $\mathrm{C}-\mathrm{H}$ groups. The presence of peaks at $1697 \mathrm{~cm}^{-1}$ and $1629 \mathrm{~cm}^{-1}$ are because of the existence of $\mathrm{C}=\mathrm{O}$ and $\mathrm{C}=\mathrm{C}$ respectively. The peak at $1396 \mathrm{~cm}^{-1}$ signifies the bending vibration of $\mathrm{C}-\mathrm{H}$. All these results related to the FTIR peaks are in agreement with the previously reported articles. ${ }^{6,25}$ 

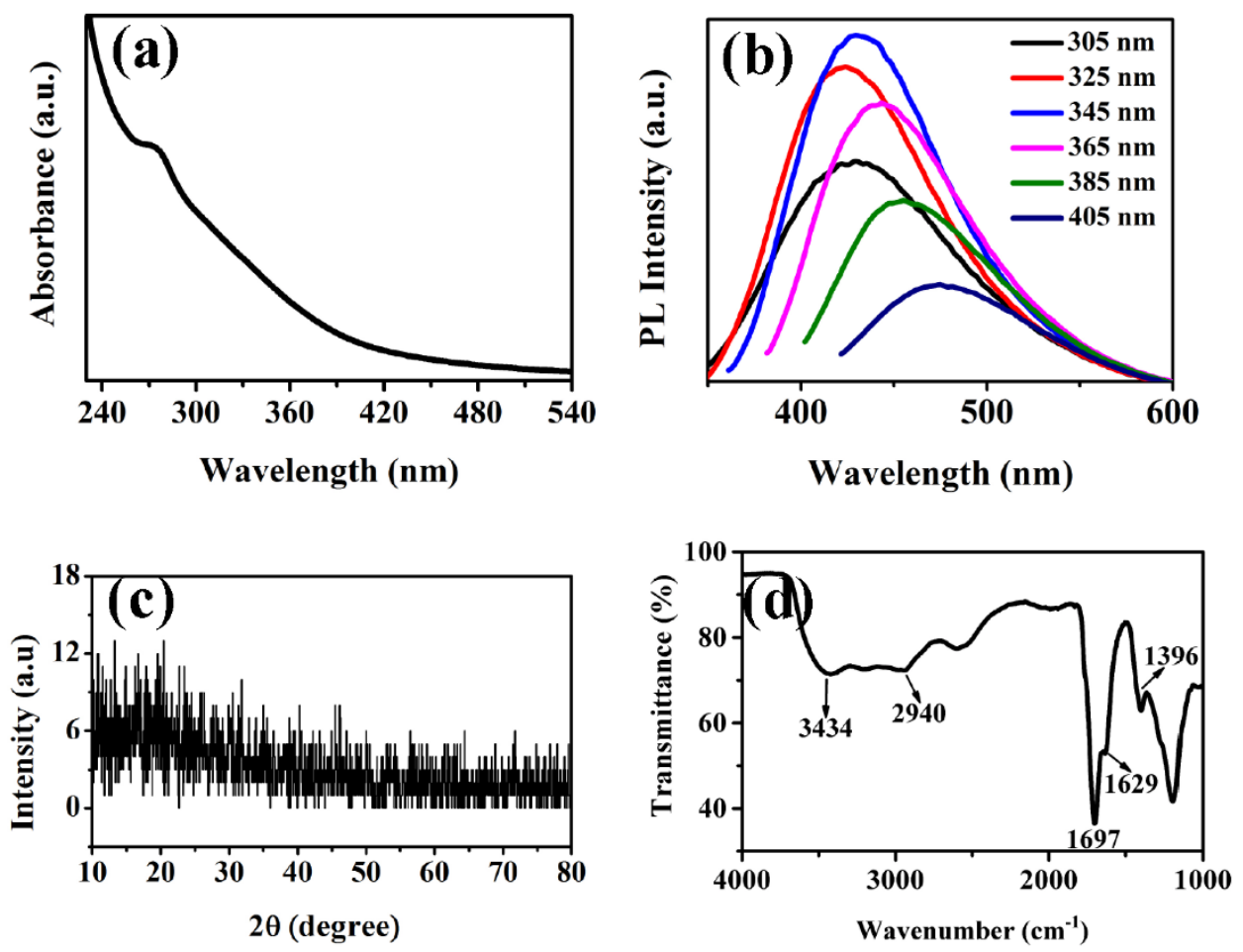

Figure 1. (a) UV-Vis spectrum. (b) PL insity at different excitation wavelength. (c) XRD plot and (d) FTIR spectrum of the synthesized CDs.

We used the photoluminescent property of $\mathrm{CD}$ to detect $\mathrm{Cr}^{6+}$ in water sample. Figure $2 \mathrm{a}$ shows the photluminescence (PL) intensity decay of $\mathrm{CD}$ solution along with the addition of $^{\mathrm{Cr}^{6+}}$ in the solution. This observation related to the decrease in PL intensity can be attributed to that, the $\mathrm{Cr}^{6+}$ metal ions can effectively quench the photoluminescence of CDs. This quenching is attained by electron transfer or energy transfer. ${ }^{24,27}$ In this figure, the decrease in PL intensity along with the increase in $\mathrm{Cr}^{6+}$ concentration dictates the sensitivity of the CDs towards different concentration of $\mathrm{Cr}^{6+}$. This photoluminescence quenching data trails the Stern-Volmer equation. The mechanism is either static or dynamic and the equation is as follows:

$$
\frac{F_{0}}{F}-1=K_{S V}[M]
$$

Where, $[M]$ denotes the metal ion concentration (here, $\mathrm{Cr}^{6+}$ ), $F_{0}$ and $F$ denote the PL intensities of CDs at $427 \mathrm{~nm}$ in the absence and presence of $\mathrm{Cr}^{6+}$ ions and $K_{S V}$ is the Stern-Volmer quenching coefficient. Figure $2 \mathrm{~b}$ shows the Stern-Volmer plot for $\mathrm{Cr}^{6+}$ ions and it is observed that there is a very good linear fit with a correlation coefficient $\left(\mathrm{R}^{2}\right)$ of 0.98169 and also this sensing system was detecting $\mathrm{Cr}^{6+}$ at concentrations as low as $0.012 \mu \mathrm{M}$. As far as the quenching of PL due to the presence of $\mathrm{Cr}^{6+}$ is concerned, it can be explained as a non-radiative recombination of electron-hole pair by energy or electron transfer process. This is due to the presence of vacant $d$ orbital and low lying $d-d$ transition state ${ }^{28}$ which ultimately helps in photoluminescence quenching of CDs. 

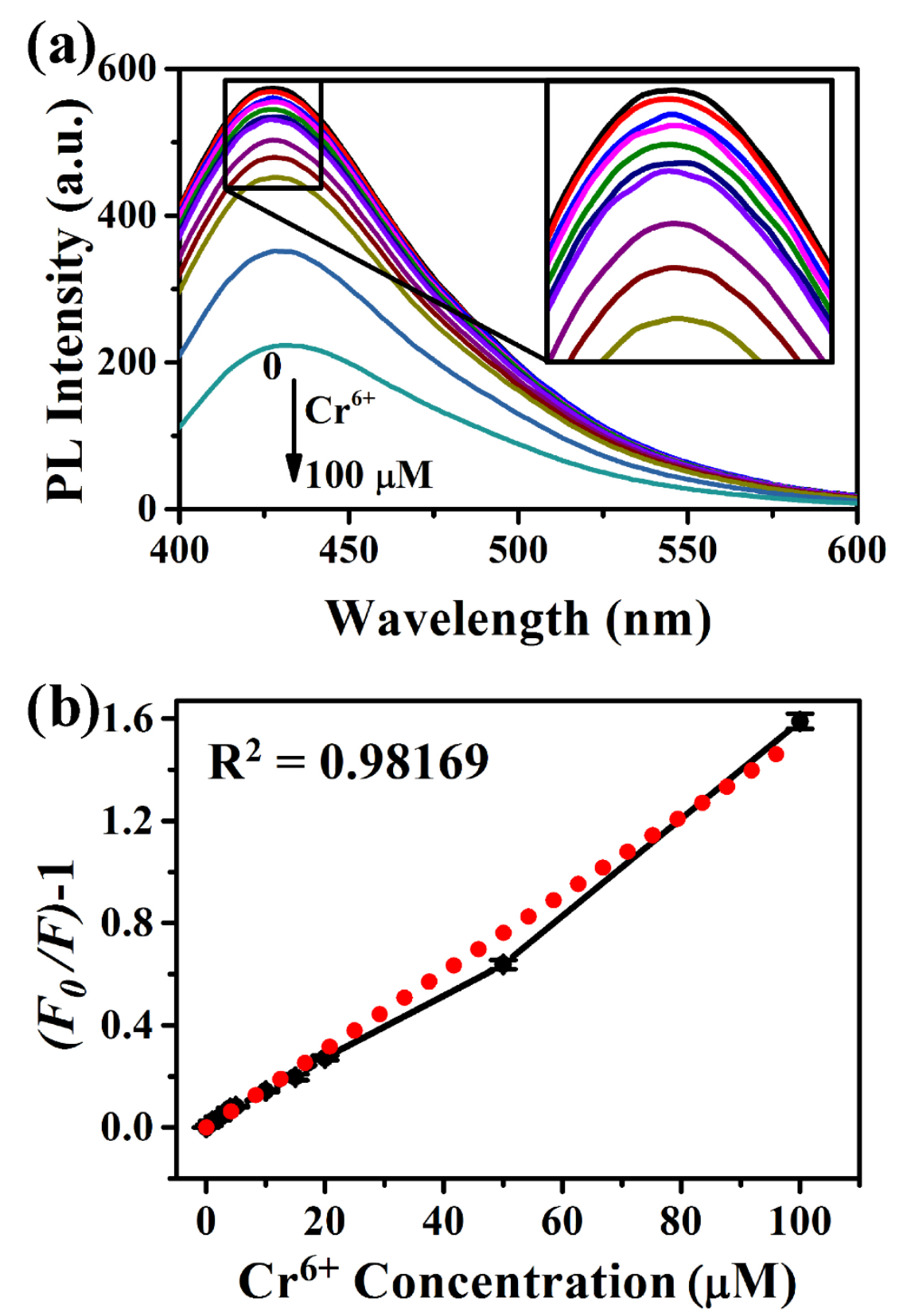

Figure 2. (a) PL spectra of CD solution in the presence of different Cr6+ concentrations (from top to bottom: $0,0.5,1,2,3,4,5,10,15,20,50$ and $100 \mu \mathrm{M})$. (b) Linearly fitted Stern-Volmer plot for the quenching of PL of CD with Cr6+. (Excitation wavelength: $345 \mathrm{~nm} ; \mathrm{F}$ and $\mathrm{F}_{0}$ are the photoluminescence intensities of CD solution at $427 \mathrm{~nm}$ in the presence and absence of Cr6+ respectively. The error bars represent the standard deviation value considering three different measurements).

The feasibility of the sensing system towards $\mathrm{Cr}^{6+}$ ions were checked using real sample water. For this, we used tannery water to check the sensitivity of the CDs. First we characterized the tannery water using UV-Vis spectrophotometer to check whether $\mathrm{Cr}^{6+}$ ions were present in the sample water or not. It was found out that the sample did not have $\mathrm{Cr}^{6+}$ ions in it, instead it was containing $\mathrm{Cr}^{3+}$ ions. Figure $3 \mathrm{a}$ is the absorbance plots of $\mathrm{Cr}^{6+}$ ion solution at different known concentration and figure $3 \mathrm{~b}$ is the corresponding calibration curve of the $\mathrm{Cr}^{6+}$ ion solutions. But incase of figure 3c (black line), we do not observe any significant peak at $351.5 \mathrm{~nm}$ 
wavelength which indicates the absence of $\mathrm{Cr}^{6+}$ ions in the collected tannery sample water. To convert the $\mathrm{Cr}^{3+}$ ions to $\mathrm{Cr}^{6+}$ ions we used the method which was reported by Rodman et al. ${ }^{23}$ The red line in figure $3 \mathrm{c}$ is the absorbance peak of oxidized tannery water. The appearance of a sharp peak at $351.5 \mathrm{~nm}$ wavelength confirms the transition of $\mathrm{Cr}^{3+}$ to $\mathrm{Cr}^{6+}$ ions. The sensitivity and selectivity of our synthesized CDs was examined using this oxidized tannery water. Figure $3 \mathrm{~d}$ the normalized PL intensity of blank CD solution and CD solution with oxidized tannery water. Here, we can observe that the system has detected the presence of $\mathrm{Cr}^{6+}$ in the solution. It is calculated from the plot that, the photoluminescence decay is around $60 \%$ after adding the oxidized tannery water.
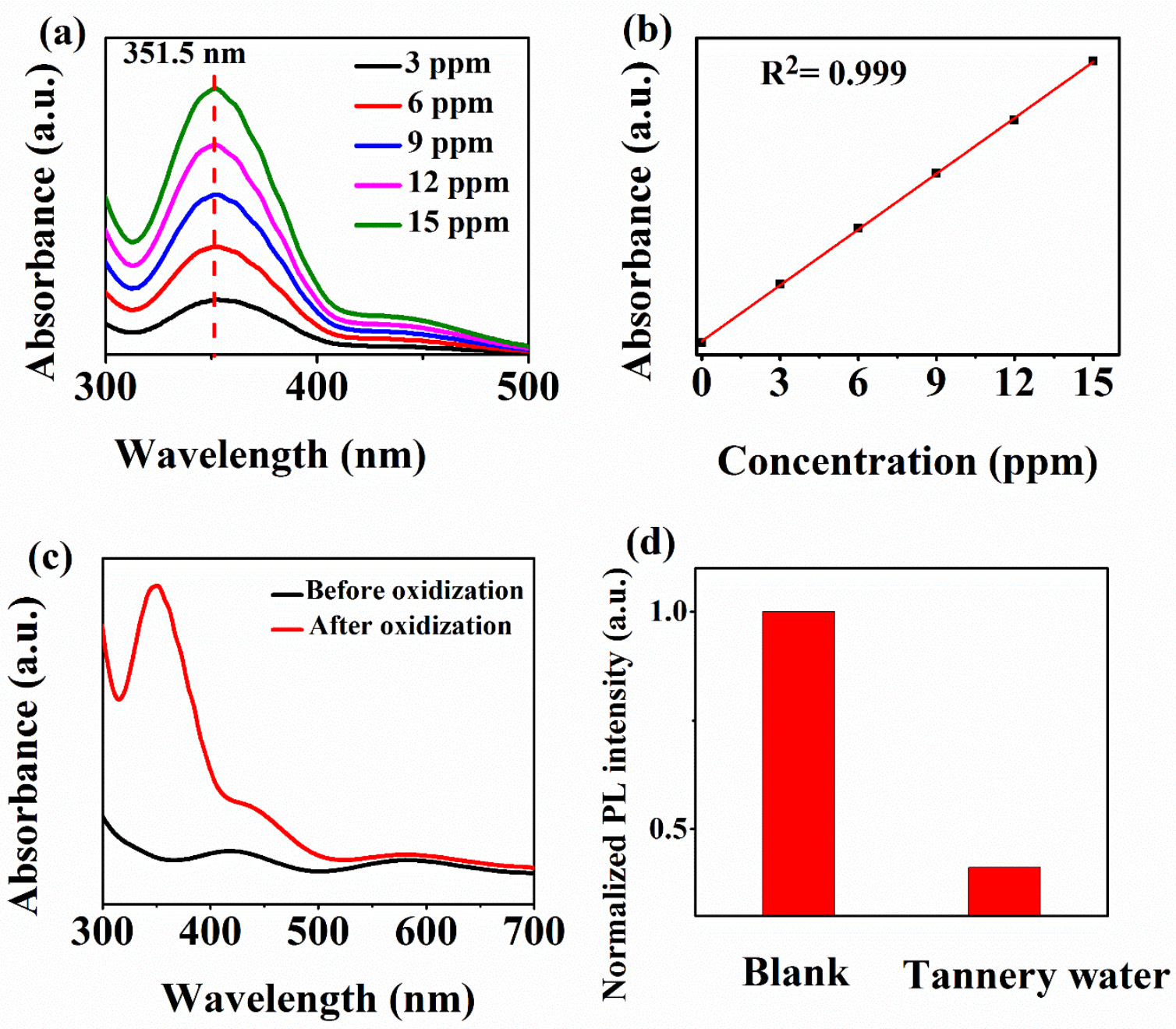

Figure 3. (a) Absorbance of $\mathrm{Cr}^{6+}$ solution of known concentration. (b) Calibration curve. (c) Absorbance of tannery water before and after oxidization. (d) Normalized PL intensity plot of blank CD solution and CD solution with oxidized tannery water.

\section{Conclusions:}

In this study, a facile synthesis of CDs from potato was developed. The synthesized CDs were found out to be water soluble which was presumably considered because of the presence of the functional groups $\mathrm{OH}$ and $\mathrm{COOH}$, detected in FTIR analysis. Thus synthesized CDs were showing noticeable photoluminescence property with a QY of $6.08 \%$ at $345 \mathrm{~nm}$. This luminescence property of the synthesized CDs was utilized to detect $\mathrm{Cr}^{6+}$ ions in water sample and this heavy metal detection quality of the CDs was further explored to check the sensing 
feasibility in case of real samples. For this, we used tannery waste water sample and we checked the $\mathrm{Cr}^{6+}$ ion sensitivity using the thus synthesized CDs. It was observed that the sensing quality of our system is quite well as it was found out that the photoluminescence decay after adding the tannery (oxidized) water sample was as high as around $60 \%$.

\section{References}

1. Sahu, S.; Behera, B.; Maiti, T. K.; Mohapatra, S. Simple one-step synthesis of highly luminescent carbon dots from orange juice: application as excellent bio-imaging agents. Chemical Communications 2012, 48, 8835-8837.

2. Xu, X.; Ray, R.; Gu, Y.; Ploehn, H. J.; Gearheart, L.; Raker, K.; Scrivens, W. A. Electrophoretic Analysis and Purification of Fluorescent Single-Walled Carbon Nanotube Fragments. Journal of the American Chemical Society 2004, 126, 12736-12737.

3. Hola, K.; Bourlinos, A. B.; Kozak, O.; Berka, K.; Siskova, K. M.; Havrdova, M.; Tucek, J.; Safarova, K.; Otyepka, M.; Giannelis, E. P.; Zboril, R. Photoluminescence effects of graphitic core size and surface functional groups in carbon dots: $\mathrm{COO}-$ induced red-shift emission. Carbon 2014, 2014, 279-286.

4. Zhang, P.; Xue, Z.; Luo, D.; Yu, W.; Guo, Z.; Wang, T. Dual-Peak Electrogenerated Chemiluminescence of Carbon Dots for Iron Ions Detection. Analytical Chemistry 2014, 86, 5620-5623.

5. Pan, J.; Sheng, Y.; Zhang, J.; Wei, J.; Huang, P.; Zhang, X.; Feng, B. Preparation of carbon quantum dots/ $\mathrm{TiO} 2$ nanotubes composites and their visible light catalytic applications. Journal of Materials Chemistry A 2014, 2, 18082-18086.

6. Shen, J.; Shang, S.; Chen, X.; Wang, D.; Cai, Y. Facile synthesis of fluorescence carbon dots from sweet potato for $\mathrm{Fe}^{3+}$ sensing and cell imaging. Materials Science and Engineering C 2017, 76, 856-864.

7. Vandarkuzhali, S. A. A.; Jeyalakshmi, V.; Sivaraman, G.; Singaravadivel, S.; Krishnamurthy, K. R.; Viswanathan, B. Highly fluorescent carbon dots from Pseudo-stem of banana plant: Applications as nanosensor and bio-imaging agents. Sensors and Actuators B: Chemical 2017, 252, 894-900.

8. Song, Y.; Zhu, S.; Yang, B. Bioimaging based on fluorescent carbon dots. RSC Advances 2014, 4, 27184-27200.

9. Mao, L.-H.; Tang, W.-Q.; Deng, Z.-Y.; Liu, S.-S.; Wang, C.-F.; Chen, S. Facile Access to White Fluorescent Carbon Dots toward Light-Emitting Devices. Industrial \& Engineering Chemistry Research 2014, 53, 6417-6425.

10. Lu, W.; Qin, X.; Liu, S.; Chang, G.; Zhang, Y.; Luo, Y.; Asiri, A. M.; Al-Youbi, A. O.; Sun, X. Economical, Green Synthesis of Fluorescent Carbon Nanoparticles and Their Use as Probes for Sensitive and Selective Detection of Mercury(II) Ions. Analytical Chemistry 2012, 84, 5351-5357.

11. Sen, L.; Jingqi, T.; Lei, W.; Yingwei, Z.; Xiaoyun, Q.; Yonglan, L.; M., A. A.; O., A. Y. A.; Xuping, S. Hydrothermal Treatment of Grass: A Low-Cost, Green Route to NitrogenDoped, Carbon-Rich, Photoluminescent Polymer Nanodots as an Effective Fluorescent Sensing Platform for Label-Free Detection of Cu(II) Ions. Advanced Materials 2012, 24, 2037-2041.

12. Zhou, L.; Lin, Y.; Huang, Z.; Ren, J.; Qu, X. Carbon nanodots as fluorescence probes for rapid, sensitive, and label-free detection of $\mathrm{Hg} 2+$ and biothiols in complex matrices. Chemical Communications 2012, 48, 1147-1149.

13. Zhao, S.; Lan, M.; Zhu, X.; Xue, H.; Ng, T.-W.; Meng, X.; Lee, C.-S.; Wang, P.; Zhang, W. Green Synthesis of Bifunctional Fluorescent Carbon Dots from Garlic for Cellular 
Imaging and Free Radical Scavenging. ACS Applied Materials \& Interfaces 2015, 7, 17054-17060.

14. Huang, H.; Xu, Y.; Tang, C.-J.; Chen, J.-R.; Wang, A.-J.; Feng, J.-J. Facile and green synthesis of photoluminescent carbon nanoparticles for cellular imaging. New Journal of Chemistry 2014, 38, 784-789.

15. De, B.; Karak, N. A green and facile approach for the synthesis of water soluble fluorescent carbon dots from banana juice. RSC Advances 2013, 3, 8286-8290.

16. Liu, M.; Xu, Y.; Niu, F.; Gooding, J. J.; Liu, J. Carbon quantum dots directly generated from electrochemical oxidation of graphite electrodes in alkaline alcohols and the applications for specific ferric ion detection and cell imaging. Analyst 2016, 141, 26572664.

17. Jaiswal, A.; Ghosh, S. S.; Chattopadhyay, A. One step synthesis of C-dots by microwave mediated caramelization of poly(ethylene glycol). Chemical Communications 2012, 48, 407-409.

18. Bu, L.; Peng, J.; Peng, H.; Liu, S.; Xiao, H.; Liu, D.; Pan, Z.; Chen, Y.; Chen, F.; He, Y. Fluorescent carbon dots for the sensitive detection of $\mathrm{Cr}(\mathrm{vi})$ in aqueous media and their application in test papers. RSC Advances 2016, 6, 95469-95475.

19. López, C.; Zougagh, M.; Algarra, M.; Rodríguez-Castellón, E.; Campos, B. B.; Esteves da Silva, J. C. G.; Jiménez-Jiménez, J.; Ríos, A. Microwave-assisted synthesis of carbon dots and its potential as analysis of four heterocyclic aromatic amines. Talanta 2015, 132, 845850.

20. Sk, M. P.; Chattopadhyay, A. Induction coil heater prepared highly fluorescent carbon dots as invisible ink and explosive sensor. RSC Advances 2014, 4, 31994-31999.

21. Sinha, R.; Bidkar, A. P.; Rajasekhar, R.; Ghosh, S. S.; Mandal, T. K. A Facile Synthesis of Nontoxic Luminescent Carbon Dots for Detection of Chromium and Iron in Real Water Sample and Bio-imaging. The Canadian Journal of Chemical Engineering 2019, Accepted.

22. Goswami, U.; Dutta, A.; Raza, A.; Kandimalla, R.; Kalita, S.; Ghosh, S. S.; Chattopadhyay, A. Transferrin-Copper Nanocluster-Doxorubicin Nanoparticles as Targeted Theranostic Cancer Nanodrug. ACS Applied Materials \& Interfaces 2018, 10, 3282-3294.

23. Rodman, D. L.; Carrington, N. A.; Xue, Z.-L. Conversion of chromium(III) propionate to chromium(VI) by the Advanced Oxidation Process: Pretreatment of a biomimetic complex for metal analysis. Talanta 2006, 70, 668-675.

24. Tyagi, A.; Tripathi, K. M.; Singh, N.; Choudhary, S.; Gupta, R. K. Green synthesis of carbon quantum dots from lemon peel waste: applications in sensing and photocatalysis. RSC Advances 2016, 6, 72423-72432.

25. Dubey, P.; Sonkar, S. K.; Majumder, S.; Tripathi, K. M.; Sarkar, S. Isolation of water soluble carbon nanotubes with network structure possessing multipodal junctions and its magnetic property. RSC Advances 2013, 3, 7306-7312.

26. Baker Sheila , N.; Baker Gary, A. Luminescent Carbon Nanodots: Emergent Nanolights. Angewandte Chemie International Edition 2010, 49, 6726-6744.

27. Guo, Y.; Zhang, L.; Zhang, S.; Yang, Y.; Chen, X.; Zhang, M. Fluorescent carbon nanoparticles for the fluorescent detection of metal ions. Biosensors and Bioelectronics 2015, 63, 61-71.

28. Yejee, H.; Youngmin, Y.; Yong-Min, L.; Wonwoo, N. Double Action: Toward Phosphorescence Ratiometric Sensing of Chromium Ion. Advanced Materials 2012, 24, 2748-2754. 


\title{
Magneto-Electrodeposition of $\mathrm{CoFe}_{2} \mathrm{O}_{4}$ Nanowire Arrays
}

\author{
Nabil Labchir ${ }^{1,2^{*}}$, Abdelkrim Hannour ${ }^{2}$, Didier Vincent ${ }^{1}$, Ahmed Ihlal $^{2}$ and Mohamed Sajieddine ${ }^{3}$ \\ ${ }^{1}$ Univ Lyon, UJM-Saint-Etienne, CNRS, LabHC,UMR 5516, F-4203, Saint-etienne, France \\ nabil.labchir@univ-st-etienne.fr, didier.vincent@univ-st-etienne.fr \\ ${ }^{2}$ Univ Ibn Zohr, Faculty of Sciences Agadir, LabMER, BP 8106, Cité Dakhla, 80000, Agadir, Morocco \\ a.hannour@uiz.ac.ma, a.ihlal@uiz.ac.ma \\ ${ }^{3}$ Univ Sultan Moulay Slimane, FST, LabPM, BP 523, 23000, Béni Mellal, Morocco \\ sajieddinem@yahoo.fr
}

\begin{abstract}
In this paper, Cobalt-ferrite $\left(\mathrm{CoFe}_{2} \mathrm{O}_{4}\right)$ nanowire arrays were electrodeposited in anodic aluminium oxide (AAO) membranes. We demonstrate that the magnetic properties of the nanowires can be changed with the application of a magnetic field (MF) B during the electrochemical growth. XRD studies have shown the presence of a polycrystalline appearance for electrodeposited nanowires. The average size of $\mathrm{CoFe}_{2} \mathrm{O}_{4}$ crystallites and the lattice parameter was determined using Scherrer's formula and FullProf software, respectively. The morphology of the $\mathrm{CoFe}_{2} \mathrm{O}_{4}$ nanowires surface were analysed by scanning electron microscope (SEM). The results showed that the wire diameter vary between 200 and $220 \mathrm{~nm}$. The chemical composition of $\mathrm{CoFe}_{2} \mathrm{O}_{4}$ nanowires was investigated by energy dispersive X-ray spectrometry (EDXS). SQUID studies indicate that the nanowires electrodeposited with application of a magnetic field have a coercive field $\mathrm{H}_{\mathrm{c}}$ of $800 \mathrm{Oe}$ and a squareness ratio $\mathrm{M} / \mathrm{M}_{\mathrm{S}}$ of 0.25 .
\end{abstract}

Keywords: Electrodeposition, magnetic field, $\mathrm{CoFe}_{2} \mathrm{O}_{4}$ nanowire, AAO membrane.

\section{Introduction}

Magnetic ferrite nanomaterials in the form of nanowires are the subject of intensive research due to their potential uses and applications in many areas, for example, in giant magnetoresistance devices, and in highdensity magnetic recording media [1-3]. Cobalt ferrite $\left(\mathrm{CoFe}_{2} \mathrm{O}_{4}\right)$ in its reverse spinel structure is known among the most attractive magnetic ferrites. In addition, $\mathrm{CoFe}_{2} \mathrm{O}_{4}$ is characterized by their good physical and chemical properties such as moderate saturation magnetization, significant magnetic anisotropy, good chemical stability, and remarkable mechanical hardness, which makes it a good material to manufacture magneto-optical components. the growing evolution of cobalt ferrite applications with nanometric size (nanoparticles, thin film, nanowires,..) and the control of these magnetic properties led to its development by several techniques, including coprecipitation [4], hydrothermal [5], inverse micelles [6], microemulsion [7], plasma [8], laser deposition [9], and combustion [10]. The above methods are known for their efficiency in producing magnetic nanomaterials with uniform and nanometric scale, but they have some inconvenient, as the need for more expensive and sometimes toxic reagents, a long duration, sophisticated installations, and high temperature. To overcome all these problems, we decided to move towards the technique of electrodeposition known by its ease of control, simplicity of equipment, and also by the diversity of the parameters to be regulated which can control the properties of electro-deposited material according to many researches in the literature [11-12], we noticed that the magnetic field is able to change the nature and the physical properties of deposit during the electrodeposition process, especially in the case of magnetic precursors such as iron and cobalt. In the present work, cobalt ferrite nanowires were synthetized by electrodeposition method with presence of a magnetic field (MF B).

\section{Experimental part}

Electrodeposition of $\mathrm{CoFe}_{2} \mathrm{O}_{4}$ nanowires was carried out using a three electrode electrochemical cell with an anodic aluminium oxide (AAO) membrane $\left(10^{\wedge}\right.$ pores $/ \mathrm{cm}^{2}, 50 \%$ of porosity $)$ as working electrode, a platinum wire, a saturated calomel electrode was used in all experiments as a counter electrode and a reference electrode, respectively. Before starting the deposition process, the AAO membranes were cleaned ultrasonically in acetone, and in distilled water for $10 \mathrm{~min}$. $\mathrm{CoFe}_{2}$ nanocomposite were electrodeposited from an aqueous solution of $0.04 \mathrm{M}$ of $\mathrm{CoSO} 4.7 \mathrm{H} 2 \mathrm{O}$, and $0.06 \mathrm{M}$ of $\mathrm{FeSO} 4.7 \mathrm{H} 2 \mathrm{O}$ at $27^{\circ} \mathrm{C}$. The $\mathrm{pH}$ of solutions was fixed to 
3 by Chloridric acid. Cyclic voltammetry and chronoamperometry investigations were carried by PGZ 301 Potentiostat, coupled to a computer with Voltamaster 4 software for potential control. $\mathrm{CoFe}_{2}$ nanocomposite were electrodeposited at $-1200 \mathrm{mV}$ for 50 minutes with presence of applied perpendicular magnetic field to the nanoporous membrane. Following the deposition, electrodeposited samples were annealed in air at $500{ }^{\circ} \mathrm{C}$ during $24 \mathrm{~h}$ to obtain the $\mathrm{CoFe}_{2} \mathrm{O}_{4}$ phase. Magneto-Electrodeposited/annealed $\mathrm{CoFe}_{2} \mathrm{O}_{4}$ nanowires was analyzed by DRX, SEM (EDXS), , and SQUID.

\section{Results and Discussion}

The synthesis of nanocomposite is carried out in potentiostatic mode, by applying a constant potential -1200 $\mathrm{mV}$ between the working electrode and the reference electrode. Figure 1 shows the variation of the current density measured as a function of time for the reduction of the $\mathrm{Co}^{2+}$ and $\mathrm{Fe}^{2+}$ ions into $\mathrm{Al}_{2} \mathrm{O}_{3}$ alumina membranes pores under the conditions described above.

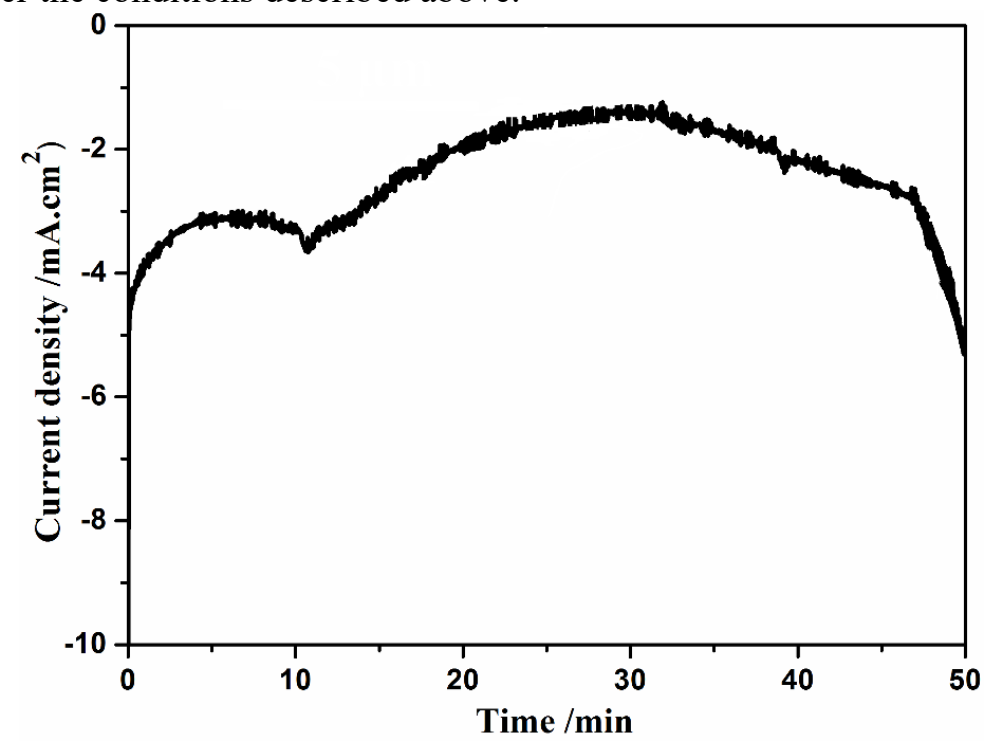

Fig. 1: Chronoamperometry curve for the magneto-deposition of $\mathrm{CoFe}_{2}$ nanocomposite.

We can distinguish 3 characteristic phases of the evolution of deposit. The first phase consists of a transitional regime for the establishment of the current density. It stabilizes in less than 10 minutes for the solution and the membrane used. The second phase corresponds to the filling of the pores of the membrane and leads to a relatively constant current-density. However, in phase 2 we note a variation of the current density which increase slightly before decreasing, this is attributed to the inhomogeneous transfer of ions into the pores. The third phase is associated with a sudden increase of the current density which corresponds to the synthesis of $\mathrm{CoFe}_{2}$ outside the membrane after complete filling of the pores. This rapid increase in current can therefore be explained by a significant increase in the working electrode area.

Micro-structural analysis and spinel phase identification was obtained from X-ray diffraction data. Figure 2 shows the X-ray diffraction spectrum which confirms that all peaks observed correspond to cobalt ferrite. The mesh parameter and the crystallites size are calculated using the Scherrer's equation and the Fullprof software [13]. 


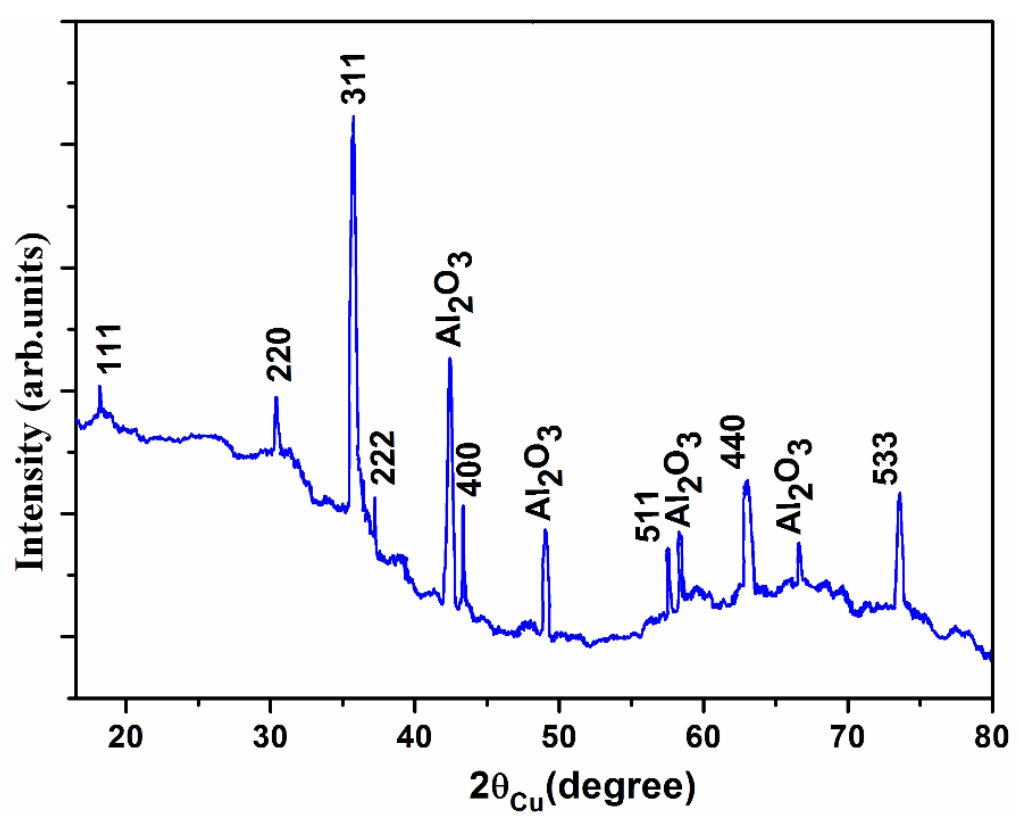

Fig. 2: X-ray diffraction of $\mathrm{CoFe}_{2}$ nanocomposite after an annealing in $500 \mathrm{C}$ during $24 \mathrm{~h}$.

Table 1: Structural parameters of $\mathrm{CoFe}_{2} \mathrm{O}_{4}$ nanowires calculated from X-ray data.

\begin{tabular}{|c|c|c|c|c|}
\hline Name & Space group & $\mathrm{a} / \AA$ & Cell volume $/ \AA^{3}$ & $\mathrm{D}$ (crystallite size) $/ \mathrm{nm}$ \\
\hline $\mathrm{CoFe}_{2} \mathrm{O}_{4}$ & $\mathrm{Fd} \overline{\mathbf{3}} \mathrm{m}$ & 8.38 & 588.48 & 20 \\
\hline
\end{tabular}

It is well observed in Figure 3 (a) the SEM of sample synthesized by the magneto-electrodeposition after their dissolution in $1 \mathrm{M} \mathrm{NaOH}$, the nanowires presented in the sample having a diameter varies from 200 to 220 nm.

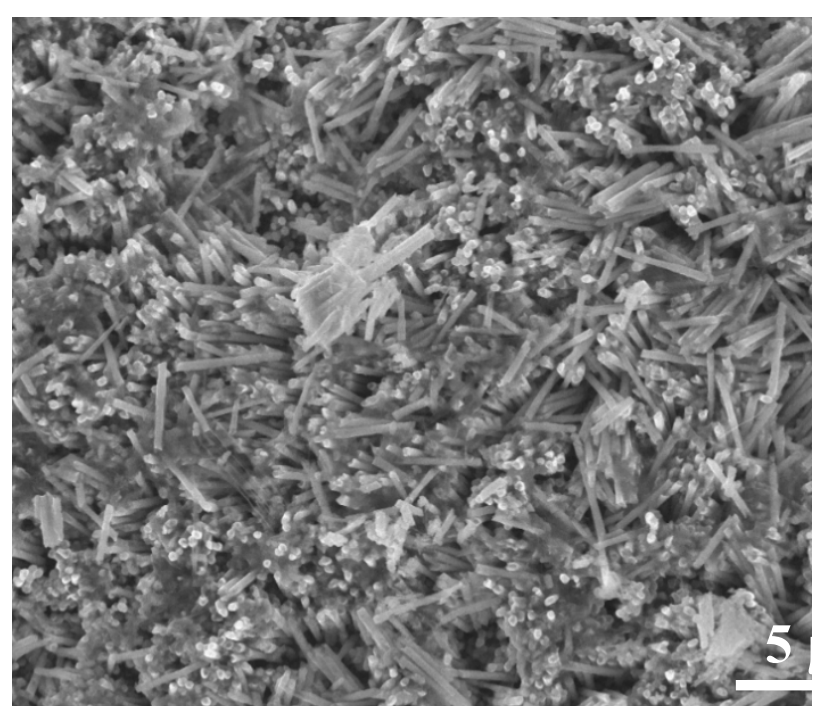




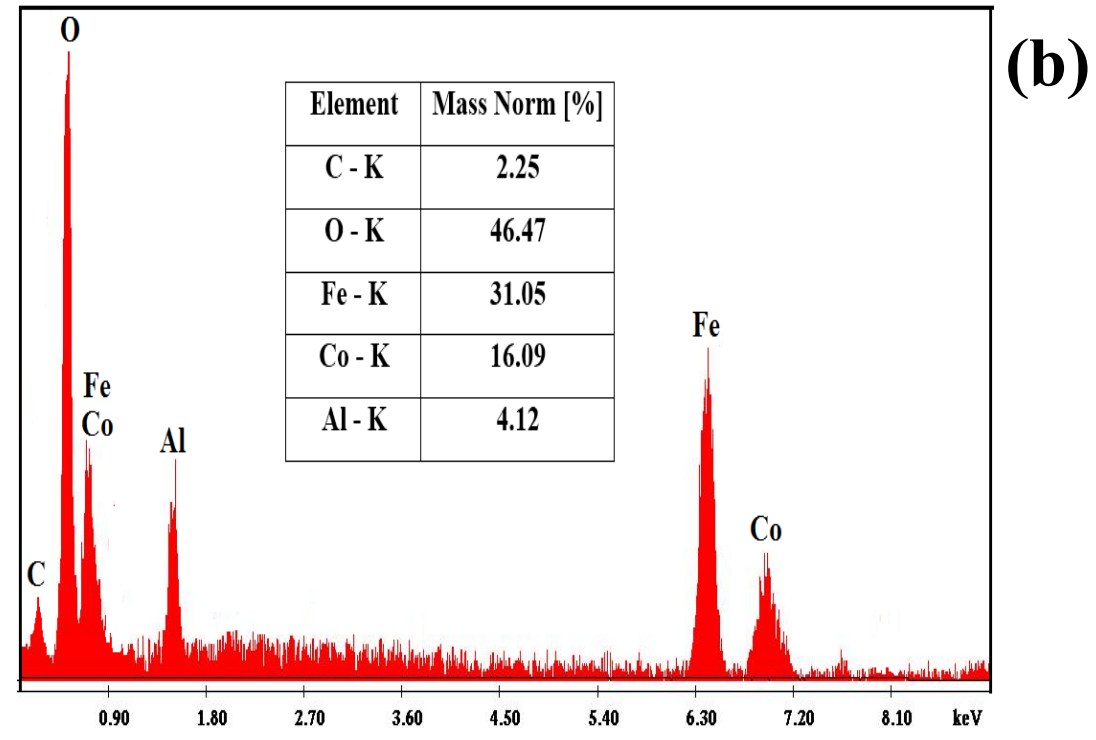

Fig. 3: SEM image and the attached EDXS spectrum of magneto-electrodeposited $\mathrm{CoFe}_{2} \mathrm{O}_{4}$ nanowires.

The EDXS spectrum and the attached table (figure $3(\mathrm{~b})$ ) for magneto-electrodeposited $\mathrm{CoFe}_{2} \mathrm{O}_{4}$ nanowires showed that the sample is composed from cobalt ferrite elements such as $\mathrm{Co}, \mathrm{Fe}$, and $\mathrm{O}$.

The Magnetic response of the $\mathrm{CoFe}_{2} \mathrm{O}_{4}$ nanowires electrodeposited with and without presence of MF B were investigated by Superconducting Quantum Interference Device (SQUID) magnetometer at room temperature (Figure 4).

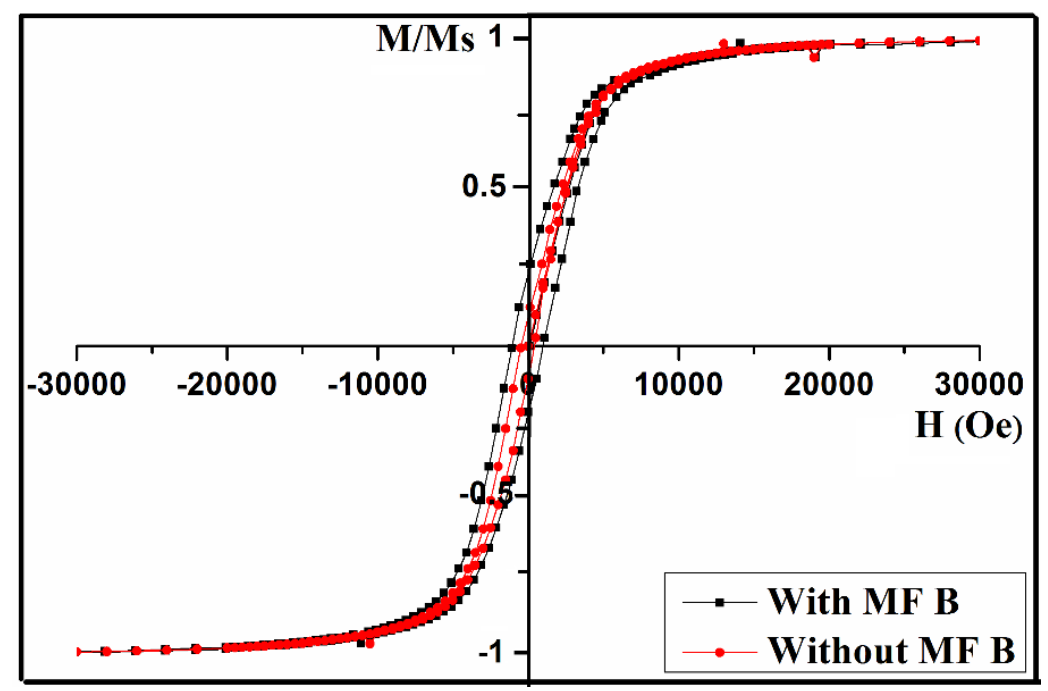

Fig. 4: Hysteresis loops for $\mathrm{CoFe}_{2} \mathrm{O}_{4}$ nanowires measured with the field applied parallel to the wire axe.

From the obtained hysteresis loops measured in the 100 Oe applied magnetic field, the Coercivity $\left(H_{c}\right)$ and the squareness $M / M_{s}$ were determined. The measured magnetic parameters were: $H_{c}=400$ and $800 \mathrm{Oe}, M / M_{s}=$ 0.12 and 0.25 for the synthesized nanowires without and with applied perpendicular MF B, respectively. According to the obtained $H_{C}$ and $M / M_{S}$ values from the magnetic hysteresis loops for the $\mathrm{CoFe}_{2} \mathrm{O}_{4}$ nanowires, it should be noted that the magnetic field improves the magnetic properties, this is related to the change of the chemical composition and the crystalline order created in the cobalt ferrite inside the pores of AAO membrane. The obtained results remain encouraging to thoroughly investigate in future works, other parameters such 
as the change of the pore diameter size of AAO membrane used as substrate, and also the annealing temperature.

\section{Conclusion}

Cobalt ferrite nanowires with diameter of about $200 \mathrm{~nm}$ were successively prepared by the mgnetoelectrodeposition. The surface morphology observations by SEM confirm the good making of 1D nanostructures that indicate the uniformity in size and shape of nanowires. The magneto-electrodeposited $\mathrm{CoFe}_{2} \mathrm{O}_{4}$ nanowires have a moderate squareness 0.25 and coercive field $800 \mathrm{Oe}$. As a continuation of the present work, we intend to obtain a good magnetic properties such as coercivity, saturation magnetization, and ferromagnetic resonance of the magneto-electrodeposited nanowires. The intention is to use AAO membranes with small diameters pores and test the possibility of annealing under a magnetic field.

\section{Acknowledgements}

This research was supported by PHC Toubkal 18/75 project (Campus France: 38983UH).

\section{References}

1. T. M. Whitney, J. S. Jiang, P. C. Searson, C. L. Chien, "Fabrication and magnetic properties of arrays of metallic nanowires," Science, Vol. 261, p. 1316, 1993.

2. L. Piraux, J. M. George, J. F. Despres, C. Leroy, E. Ferain, R. Legras, K. Ounadjela, A. Fert, "Giant magnetoresistance in magnetic multilayered nanowires," Appl. Phys. Lett, vol. 65, p. 2484, 1994.

3. J. M. D. Coey and C. L. Chien, "Half-Metallic Ferromagnetic Oxides," MRS Bull, vol. 28, pp. 720-724, 2003.

4. Y. Kim, D. Kim and C.S. Lee, "Synthesis and characterization of $\mathrm{CoFe}_{2} \mathrm{O}_{4}$ magnetic nanoparticles prepared by temperature-controlled coprecipitation method," Physica B, vol. 337, pp. 42-51, 2003.

5. L. J. Cote, A. S. Teja, A. P. Wilkinson and Z. J. Zhang, "Continuous hydrothermal synthesis of $\mathrm{CoFe}_{2} \mathrm{O}_{4}$ nanoparticles," Fluid Phase Equilibr, vol. 210, p 307, 2003.

6. C. T. Seip, E. E. Carpenter, C.J. O'Connor, J. Vijay and S. Li, "Magnetic properties of a series of ferrite nanoparticles synthesized in reverse micelles," IEEE Trans. Magn vol. 34, p. 1111, 1998.

7. F. Foroughi, S. A. H. Tabrizi and J. Amighian, "Microemulsion synthesis and magnetic properties of hydroxyapatite-encapsulated nano $\mathrm{CoFe}_{2} \mathrm{O}_{4}$," journal of magnetism and magnetic materials, vol. 382,pp.182-187, 2015.

8. J. S. Jung, J. H. Lim, K. H. Choi, S. L. Oh, Y. R. Kim, S. H. Lee, D. A. Smith, K. L. Stokes, L. Malkinski and C. J. O'Connor," $\mathrm{CoFe}_{2} \mathrm{O}_{4}$ nanostructures with high coercivity," JOURNAL OF APPLIED PHYSICS, vol. 97, p. 306, 2005.

9. J. Barbosa, B. G. Almeida, J. A. Mendes, A. G. Rolo, J. P. Araújo and J. B. Sousa ,'Nanogranular $\mathrm{BaTiO}_{3}-\mathrm{CoFe}_{2} \mathrm{O}_{4}$ thin films deposited by pulsed laser ablation," Journal of Applied Physics, vol. 101, p. 09M101, 2007.

10. C. H.Yanz, Z. G. Xuf, F. X. Cheng, Z. M. Wang, L. D. Sunc, C. S. Liaoj and J. T. Jia, ” Nanophased $\mathrm{CoFe}_{2} \mathrm{O}_{4}$ prepared by combustion method, "Solid State Commun, vol. 111, p. 287, 1999.

11. Y. Yu, Z. Song, H. Ge, G. Wei and L. Jiang," Effects of Magnetic Fields on the Electrodeposition Process of Cobalt," Int. J. Electrochem. Sci, vol. 10, pp. 4812 - 4819, 2015.

12. H. Matsushima , T. Nohira , I. Mogi and Y. Ito, "Effects of magnetic fields on iron electrodeposition," Surface and Coatings Technology, vol. 179, pp. 245-251, 2004.

13. D. Erdem, N. S. Bingham, F. J. Heiligtag, N. Pilet, P. Warnicke, L. J. Heyderman and M. Niederberger," $\mathrm{CoFe}_{2} \mathrm{O}_{4}$ and $\mathrm{CoFe}_{2} \mathrm{O}_{4}-\mathrm{SiO}_{2}$ Nanoparticle Thin Films with Perpendicular Magnetic Anisotropy for Magnetic and Magneto-Optical Applications," Advanced Functional Mater, vol. 26, pp. 1954-1963, 2016. 


\title{
Crossbar Architecture for Memristor Based Memory Simulation
}

\author{
Meenal Negi, Seshan Srinivasan, and Lili He \\ San Jose State University \\ Department of Electrical Engineering \\ San Jose, CA 95192-0084, USA
}

\begin{abstract}
Crossbar architectures can be used to construct large memory arrays. The non-volatile characteristic nature of Memristor allows the crossbar design to store values. For this paper, the authors have considered simulation of a $4 \times 4$ crossbar array thereby reading and writing 16 bits to the memory subsystem. The model for memristor and the memristive crossbar for this paper were generated in SIMULINK software. The Memristor was characterized with initial parameters using a MATLAB function. The memory subsystem signals for read and write were generated using MATLAB.
\end{abstract}

Keywords: memristor, crossbar architecture, Simulink, MATLAB, simulation

\section{Introduction}

Memristors are considered as the fourth basic passive element after resistors, capacitors and inductors. Memristors are passive nano-electronic devices similar to resistors. Memristors are usually followed up with active circuit elements such as field effect transistors to boost or amplify the signals. Memristors exhibit nonvolatility characteristic. In this project, a $4 \times 4$ crossbar memory array was simulated using Memristors in Simulink software. Crossbar architectures are considered the easiest in terms of fabrication. Crossbar architecture have good noise tolerance capabilities. The amount of power consumed grows linearly with the size of the crossbar matrix [1-2] due to alternate current paths thus lowering the overall effective resistance.

Crossbar switches are created when two wires overlap each other at any angle other than zero. In a $4 \times 4$ crossbar matrix, there would be total of 16 intersections. Each intersection contains 1 memristor. A $4 \times 4$ crossbar matrix is capable of storing 16 bits. Read and Write signals required for reading/ writing data into and from the crossbar were generated using MATLAB software. There are lot of advantages to use the crossbar array of memristors in ultra-high density memory modules. In current market scenario, most of the memory read / write cycle happens through a DMA controller between the memory module and the CPU systems in case of CMOS transistors. This requires additional circuitry for DMA controller. This additional circuitry takes up additional place on the silicon.

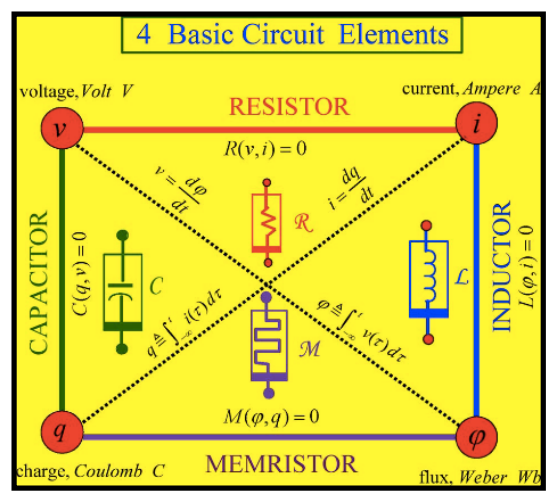

Figure 1: Four basic passive circuit elements [3]

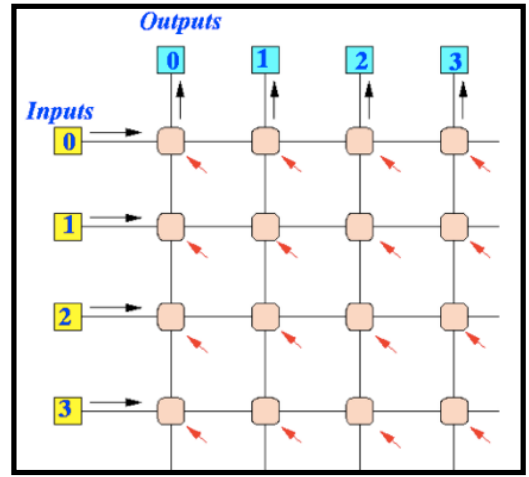

Figure 2: 4x4 Crossbar [4]

Adding to that, the additional DMA block functions in an independent clock domain. The memory module functions in a different clock domain than that of DMA controller. The memory modules usually have their own clock tree and the read/write operation takes place according to this clock signal. When there are multiple clock domains present on a chip that requires to be controlled by an external clock synchronization tree. This adds to the additional burden on the silicon of the chip. Also, these additional circuitries require more power. 
As the number of circuitries increases, the number of power and ground pin on the silicon increases. This may result in multiple problems like for example, ground bounce if the ground pins are not properly grounded. Additional transistors and resistors are used to prevent ground bounce.

The data is usually written to the DMA controller first. The DMA controller then writes the data to the memory block. Same is true for read. Data is read from the memory and written on to the DMA and the user requested data is read back from the DMA. Since the read/ write cycles are through a DMA controller, the maximum speed of memory cannot be leveraged and is limited by the speed of read/ write to the DMA controller. In case of memristors, these devices can directly be embedded on to the processor. This reduces the read/write time cycles. Thus, the memristor based memory circuit works faster than the traditional CMOS memory circuits. Memristors do not need an intermediate DMA controller to read or write the data to/ from the memory. Thus, the maximum speed of the memristive device can be leveraged.

Memristors are sized in the order of $\mathrm{nm}$. Also, since there is no need for a DMA controller on chip for a memristor based memory module, it saves a lot of silicon area on the chip. There will be no clock synchronization block required for memristors. There are no additional components/circuitries involved in the process to be taken care of like for example, ground bounce in case of CMOS transistors. Hence using memristor based memories, the memory module can be made denser and faster. There are lot of other advantages as compared to traditional circuits like reduction in area on silicon, no additional clock synchronization blocks, no additional DMA controller to read/write data from the device. The research ongoing in this domain has proposed many potential applications in Memristors. There are a few recent research topics covered as a part of this paper. The authors have explained these in detail in later sections of the paper. Memristor based memory arrays can be used to design a non-destructive read/write circuit. Even after reading the data multiple times, stored data will be intact. The normalized width is varied to store the data. Non-Linear characteristic of memristor can be utilized to develop a reservoir computing method. It is an essential element of Recurrent Neural Networks. The memristor are given a feedback input. The change in output is observed between two levels of doping surface in a memristor. The current-voltage characteristic $\mathrm{f}$ memristor can be used for pattern recognition purpose. Array of memristors collectively learn patterns using competitive learning. Memristor can be used to create analog Integrated Circuits for any application. The charge flux dependency of memristor can be utilized to have more precise analog circuits.

Memristor was proposed to be an element of high importance in future hardware devices. Thus, the security leaks in memristor need to be addressed. Circuits should be able to protect itself from hardware trojans to avoid malicious modifications. The non-linear behavior parameters can be used to have a physically unclonable circuitry. Denial of service is another kind of attack which a hardware is susceptible to. The tendency of memristor to include current in adjacent device can be utilized to detect any denial of service attack. The early model [1] suggested that memristor should be considered as a part of fundamental elements of circuit along with resistors, inductors and capacitors. Memristors are defined in terms of relationship between charge and flux-linkage [1]. The value of memristance depends on the integral of current flowing through the memristor from time $t=-\infty$ to time $t=t_{0}$. Therefore, even though memristor displays characteristics similar to that of resistors at $t_{0}$, its conductance depends on the past current passed through it as well. Once the memristor is applied with $v(t)$ or $i(t)$, it starts functioning as a linear time varying resistor. In few cases when $M(q)=R$ and $\mathrm{W}(\Psi)=\mathrm{G}$, it acts like time invariant resistor and the $\Psi$-q curve is linear. The first practical memristor was fabricated by HP in 2008 [3]. It was assumed the memristive devices comprised of two regions viz. doped and undoped. The total resistance of the memristor was the sum of the doped and undoped regions. All memristive devices share a common characteristic i.e. oxides are sandwiched between metals (Metal Insulator Metal or MIM). These type of devices have varying memories and are non-volatile. They have very low switching time and very efficient and highly enduring write cycles. Average number of writes that can be performed before the memory becomes unreliable ranges between $10^{9}$ to $10^{15}$. These devices have effectively very low switching power since these devices are very small. Switching power ranges between 0.1 to 1 pico J.

In today's computer architecture, memristors can be very useful. Memristors exhibit few important characteristics which are considered important for memory industry and those are high endurance, high 
reliability and low switching times to name a few. Additional memory levels can be added to the existing memory architecture. Memristors have better speed, data retention and endurance as compared to Flash memory. Memristor based memories are comparable to SRAM, DRAM for the speed of write operation. The write speed varies for memristive memories from tens of nano-seconds to hundreds of pico-seconds. Endurance can be measured by the number of writes a memory can handle without other data being corrupted. Current memory industry suffers below the $45 \mathrm{~nm}$ technology. As memristors have excellent read/write speed, durable and are scalable, memristors are a potential replacement for Flash memories, SRAM and DRAM type of memories. Memristors therefore, offers a "universal - memory" idea for all memory hierarchies [5].

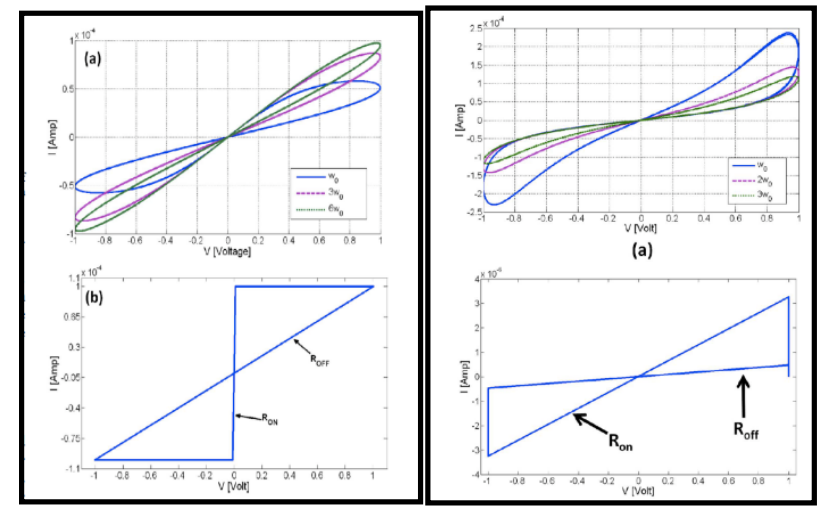

Figure 3. a). Linear Drift

b). Non-linear Drift [3]

Memristor based crossbar arrays are considered the basic building block for ultra high density memories. With high density comes the problem of high power consumption. The power consumption increases dramatically with increase in size of the crossbar size. The power draw is high because of the alternating paths for current to flow in a crossbar architecture. The alternating current paths might lead to read errors at such a high density memory module. One way to avoid the read errors and high power consumption is to divide the array in to smaller sized tiles. Each tile offers effective resistance to prevent a large power draw. This results in much denser memory modules with high accuracy of read and write cycles in a crossbar array. The proposed design in this paper is mix of high density array and one which provides transistor isolation.

In this work, we implemented a $4 \times 4$ crossbar memristive tile. Multiple such blocks can be connected together to build an array. The proposed design consists of a pulse generator block which selects a particular row to read or write the data. The design also consists of a Row decoder circuit which helps to select a particular tile for performing read or write cycles.

\section{Proposed Model and Simulation}

Apart from the row selector and pulse generator block there is a column selector circuit. Column selector circuit helps to select a particular column within a tile of memristors. During a read or write operation, the column selector processes the data selected by row circuits. The write operation is split in to two steps. The first step in the write operation is to write data ' 1 ' to the selected rows. To do this, a voltage of $V_{w} / 2$ is applied to a selected row and $-V_{w} / 2$ to the columns where data ' 1 ' needs to be written. The remaining rows are applied a voltage of 0 or in other words grounded. For writing data ' 0 ' to the select columns in a particular selected row, a voltage of $\mathrm{V}_{\mathrm{w}} / 2$ needs to be applied across global column selector.

A parallel read operation can be performed by setting the select row transistors voltages below the switching threshold. This enables the read-enable transistors and data can be read from the memristive crossbar tiles in parallel. After each write operation, a read operation was performed to validate the data written in the tile. The data written in the memristors were generated from MATLAB script randomly. +V represented a logic ' 1 ' and $-\mathrm{V}$ represented a logic ' 0 '. The data was generated randomly in MATLAB script to write or read data from the crossbar tile. In this setup, it was common to observe write errors more often than read errors because of 
the wire resistances connecting the row-selector, column-selector. However, this situation can be avoided by increasing the write voltage thereby increasing the threshold voltages across the memristive crossbar tile.
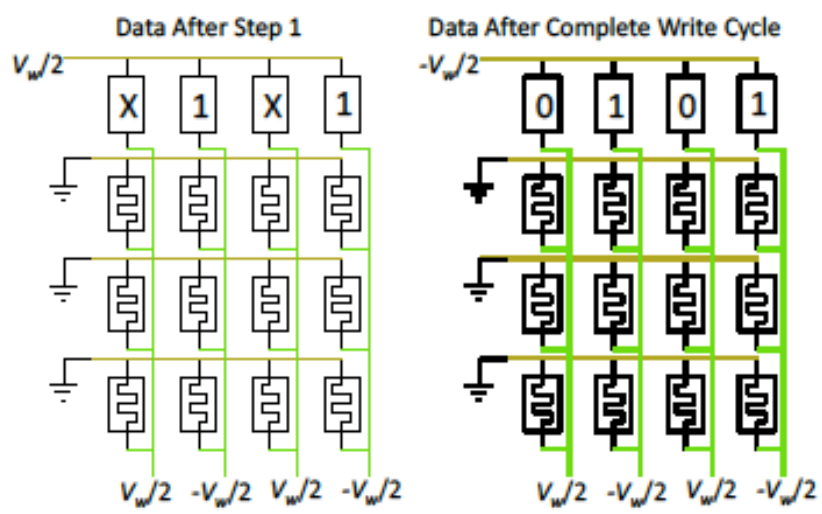

Figure 4. Write operation in a crossbar array [4]

We simulated a memristor model in MATLAB based on the theoretical values presented in [6] and [7]. The memristor model consisted of a $5 \mathrm{~nm}$ thick $\mathrm{TiO}_{2}$ and oxygen poor $\mathrm{TiO}_{2-\mathrm{x}}$ layers sandwiched between platinum (metal) plates. These $\mathrm{TiO} 2$ region creates a doped region whose resistance is lower than the undoped region. The total thickness of the memristor considered for this model is $10 \mathrm{~nm}$ of which $5 \mathrm{~nm}$ is considered as doped and the rest is undoped region. The block diagram of the mathematical model is as follows:

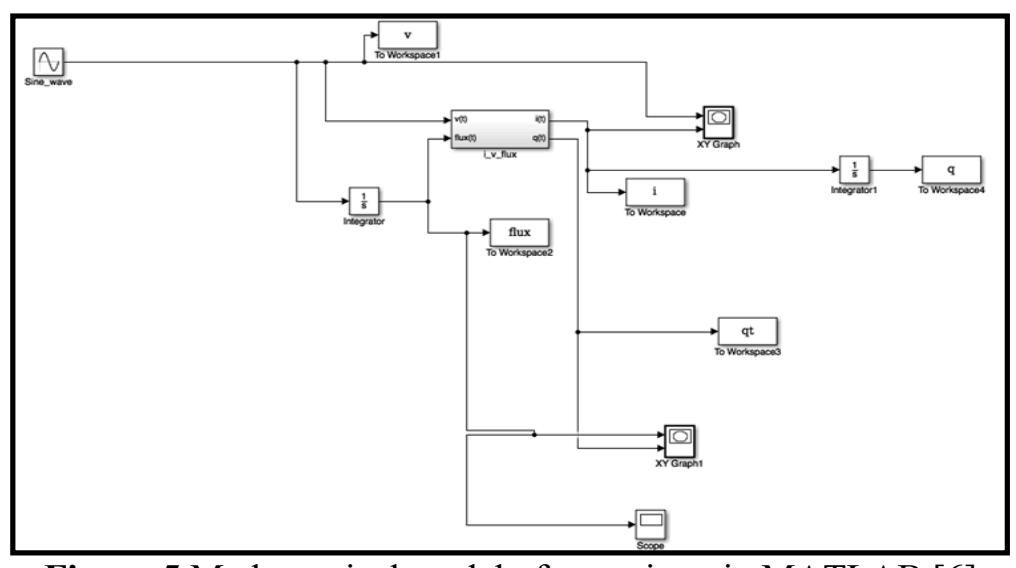

Figure 5 Mathematical model of memristor in MATLAB [6]

The boundary between the doped and undoped region is determined by the mobility constant $\mu_{\mathrm{D}}$ and the electric field across the doped region. The value for mobility was assumed to be constant and the value used for simulation was $\sim 10^{-10} \mathrm{~cm}^{2} \mathrm{~V}^{-1} \mathrm{~s}^{-1}$. R $\mathrm{ON}$ was considered as $100 \Omega$ and $\mathrm{R}_{\mathrm{OFF}}$ was considered $16 \mathrm{~K} \Omega$, where $\mathrm{R}_{\mathrm{ON}}$ is the resistance of doped region and $\mathrm{R}_{\mathrm{OFF}}$ is the resistance of undoped region. In presence of voltage $v(t)$, the current through the memristive device can be given by Kirchhoff's voltage law $v(t)=M(w) * i(t)$. 


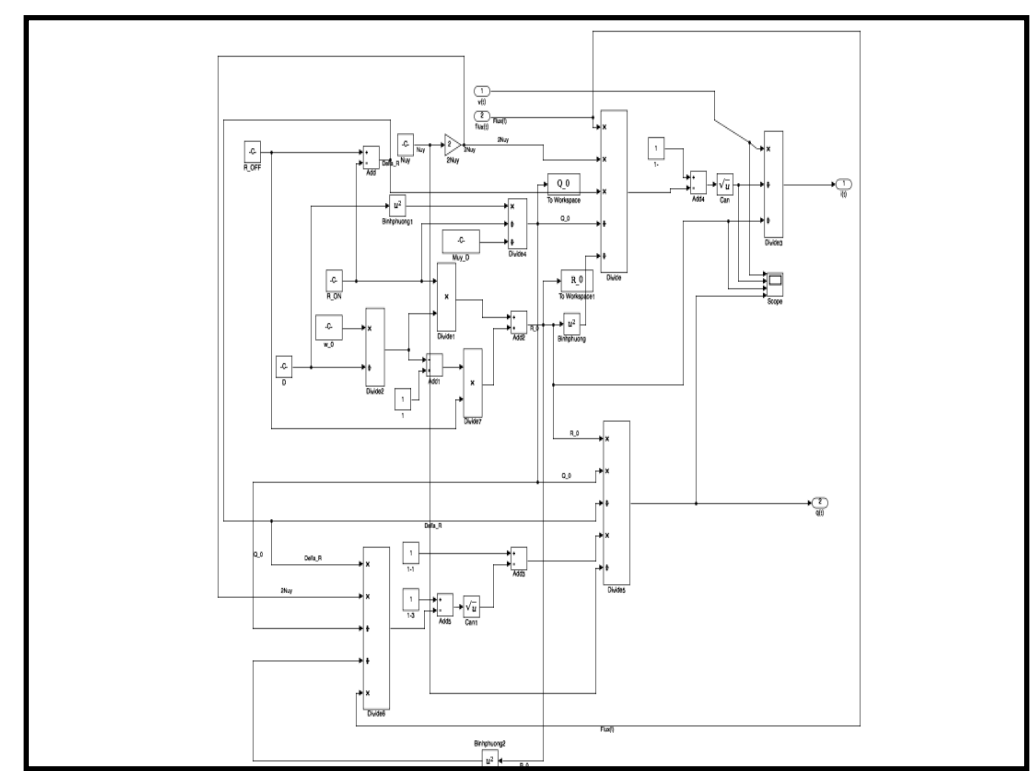

Figure 6. Mathematical model of $4 \times 4$ crossbar [6]

The size if the doped region $\mathrm{w}(\mathrm{t})$ is time dependent which proves the memristive characteristic. The drift across the dopant can cause the doped region to contract or expand. The polarity of the memristor given by $\eta$ can be between \pm 1 . When the doped region expands, the value of $\eta$ is considered to be +1 . When the doped region contracts, the value of $\eta$ is considered to be -1 . Thus, a memristor when connected in a crossbar structure shows a variation of I-V characteristics similar to that of a single memristor. The I-V characteristics is based on the frequency of applied voltage. The mobility of the dopants play an important role in the memristive characteristics exhibited by memristors. Thus, in order to have the best outcome from the memristors, the memristors should be nano-scale devices. In order to get the I-V characteristics and Charge-Flux characteristics similar to the ideal theoretical memristor crossbar, the authors have considered the total width of the memristor to be $10 \mathrm{~nm}$ and the width of the doped region to be $5 \mathrm{~nm}$ which is half of the total region. The initial $\mathrm{R}_{\mathrm{ON}}$ and $\mathrm{R}_{\mathrm{OFF}}$ values considered for this implementation are $100 \Omega$ and $16 \mathrm{~K} \Omega$ respectively.

\section{Results and Discussion}

Case $A: W<<D$

First case of simulation was when the width of doped region was considered to be much less than the total thickness. $\mathrm{w}<<\mathrm{D}$. In order to implement this, the value considered for $\mathrm{w}$ was $1 \times 10^{-12} \mathrm{~m}$ and the value for $\mathrm{D}$ was considered to be $10 \times 10^{-9} \mathrm{~m}$. With the width of doped region being so small compared to the total width (D), the switching time of memristors were very fast. This results in the violation of timings for the row and column selector circuits. These circuits did not have adequate amount of switching time thereby resulting in setup and hold time violations in most cases. For this scenario in simulation, the frequencies were adjusted such that the hold and setup times were satisfied for the row and column circuits. The reads resulted in improper data being read compared to a standard output expected. The read cycles immediately after write cycles resulted in redundant data in some cases. This redundancy was because of the faster switching times of the memristor that either the data was not written properly to the memristor or was incorrectly read back from them. Plot 1 in figure 8 represents the I-V characteristics of a memristor. Plot 2 represents the Charge v/s Flux curve. The different colors in the chart represent behavior at different frequencies. Plot 3 represents the Hysteresis curve. The different frequencies are depicted in different colors in the hysteresis curve. The ON depends on the applied frequency. 


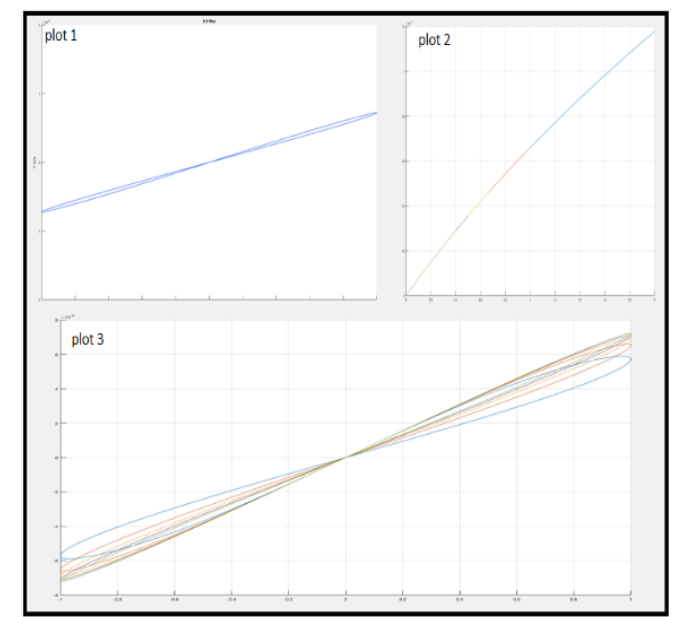

Figure 8. $w<<$ D. Plot 1: I-V curve, Plot 2: Charge/flux curve, Plot 3: Hysteresis curve at different frequencies

Case B: $W=D / 2$ (Ideal Case)

Second case of simulation was when the width of doped region was considered to be half of the total thickness. $\mathrm{w}=\mathrm{D} / 2$. This case is considered to be the ideal case for simulation of a memristive system. This case considered to be ideal because the ON and OFF times are equal for the memristors thereby enabling smooth read and write cycles. The data can be written to one row and can be parallel read from the other cell. This scenario is best for parallel processing of data. It has adequate amount of read and write cycles so that there are no timing violations. Timing violations are not specifically related to the memristors but the circuits used for selection of Row and columns as explained in previous section. The pulse generators, row selector and the column decoder have valid setup and hold time to read or write the data to / from the memristive grid. This scenario can be used to store multiple bits of data in a single memristor. This scenario provides with optimum endurance, high speed read/writes and with great accuracy. This scenario provides the simulation of memristors which are very close to the theoretical model developed by Leon Chua [1]. Plot 1 in the below figure represents the I-V characteristics of a memristor. Plot 2 represents the Charge v/s Flux curve. The different colors in the chart represent behavior at different frequencies. Plot 3 represents the Hysteresis curve. The different frequencies are depicted in different colors in the hysteresis curve. The ON depends on the applied frequency.

Case $C: W=D$

Third case of simulation was when the width of doped region was considered to be equal to the total thickness of the region. $\mathrm{w}=\mathrm{D}$. This case was simulated to realize the impact of the width of doped region being the same as the total width on read and write cycles. When the width (w) is equal to (D), the value for $\mathrm{R}_{\mathrm{ON}}$ is equal to the value of $\mathrm{R}_{\mathrm{OFF}}$. The characteristics exhibited by the array is similar to that of a linear drift memristor. In linear drift model, the boundary conditions are not considered i.e. when $\mathrm{w}=\mathrm{D}$. When current is passed through the memristor for a sufficiently large amount of time, the value of memristance is lesser than the value of resistance $\mathrm{R}_{\mathrm{ON}}$ and $\mathrm{w}(\mathrm{t})$ will be larger than $\mathrm{D}$, the total width of the memristor, which is contradictory to the physical properties of the memristor developed by HP. In this scenario, when there is a write operation taking place, redundant data gets written to the memristor because of the ON time for memristor. When this data is read back, there tends to be error reading the data written. The accuracy for reading the data written previously is relatively low in this scenario. Plot 1 in the below figure represents the I-V characteristics of a memristor. Plot 2 represents the Charge v/s Flux curve. The different colors in the chart represent behavior at different frequencies. Plot 3 represents the Hysteresis curve. The different frequencies are depicted in different colors in the hysteresis curve. The $\mathrm{ON}$ depends on the applied frequency. 


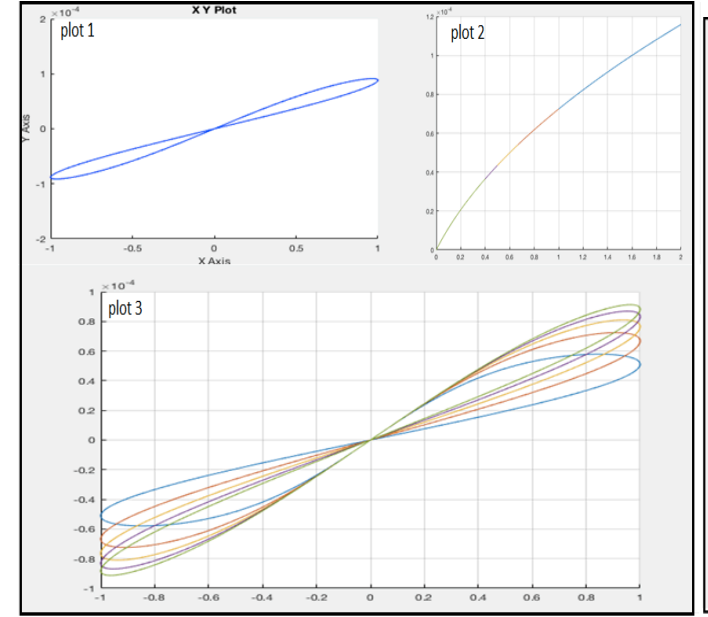

Figure 8, W = D/2

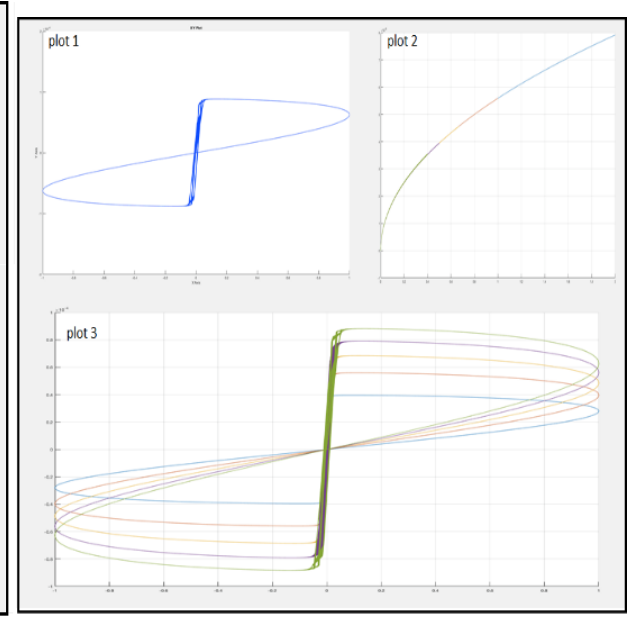

Figure 9, $\mathrm{W}=\mathrm{D}$.

Plot 1: I-V curve, Plot 2: Charge/flux curve Plot 1: I-V curve, Plot 2: Charge/flux curve, Plot 3: Hysteresis curve at different frequencies

\section{Conclusion}

Memristor, a nano scale, passive fourth element was simulated and characterized as a part of this project. Different ratio of $\mathrm{w}$ vs. D situations were simulated, and the results are discussed. The best ratio needs to consider various trade-offs. Memristors provide a major change in the way memory modules are managed in computer architecture. The proposed crossbar architecture memory using memristor aims to be denser than existing RAMs. The proposed crossbar architecture aims at reducing the area and silicon on chip thereby reducing the power consumption. The simulation results at difference width $\mathrm{w}$ vs. total doping thickness ratio w/D is critical for the potential memory performance. The existence of hysteresis indicates that the memory properties relation. Memristors could be used to design devices that will phase out transistors eventually in the memory applications.

\section{References}

1. L.O.Chua, "Memristor - the missign circuit element", IEEE Trans. Circuit Theory, Vols. CT-18, no. 5, pp. 507-519, September 1971.

2. S. J. Wolf and Y. N. Joglekar, "The elusive memristor: properties of basic electrical circuits", European Journal of Physics, Vol. 30, pp. 671-675, 2009.

3. D. B. Strukov, S. S. Gregory, D. R. Stewart and S. R. Williams, "The missing memristor found" , Nature, vol. 453, pp. 80-83, 2008.

4. P. Zhou, B. Zhao, Y. Zhang, J. Yang and Y. Chen, "MRAC: A Memristor-based Reconfigurable Framework for Adaptive Cache Replacement," The Proceeding of International Conference on Parallel Architectures and Compilation Techniques, 2011.

5. M. Elshamy, H. Mostafa, Y. H. Ghallab and M. S. Said, "Novel non-destructive read/write circuit for memristor based memory arrays," IEEE Transactions on Very Large Scale Integration Systems, vol. 23, no. 11, pp. 2648-2656, November 2015.

6. H. Y., H. G.M. and L. P., "Dynamical properties and design analysis for nonvolatile memristor memories," IEEE Trans. Circuits Syst. I, Reg. Papers, vol. 58, no. 4, pp. 724-736, April 2011.

7. M. S. Kulkarni and C. Teuscher, "Memristor-based reservoir computing," in the Proceedings of IEEE /ACM International Symposium on Nanoscale Architectures (NANOARCH), pp. 226-232, 2012.

8. S. Shin, K. Kim and S. M. Kang, "Memristor Applications for Programmable Analog ICs," IEEE Transactions on Nanotechnology, vol. 10, no. 2, pp. 266-274, March 2011.

9. S. Kvatinsky, K. Talisveyberg, D. Fliter, A. Kolodny and U. C. Weiser, "Models of Memristors for SPICE Simulations," in the Proceedings of 27th Convention of Electrical and Electronics Engineers in Israel, 2012. 


\title{
Modelling and Experimental Validation of Water Droplet Motion Influenced by Surface Acoustic Waves
}

\author{
Nurkhat Zhakiyev ${ }^{1}$, Zamart Ramazanova ${ }^{1}$, Kurbangali B Tynyshtykbayev ${ }^{1}$ and Zinetula \\ Insepov ${ }^{1,2,3^{*}}$ \\ ${ }^{1}$ Nazarbayev University, Nur-Sultan, Kazakhstan, Nurkhat.zhakiyev@nu.edu.kz \\ ${ }^{2}$ Purdue University, West Lafayette, IN, United States, zinsepov@purdue.edu \\ ${ }^{3}$ National Research Nuclear University MEPhI, Moscow, Russian Federation
}

\begin{abstract}
In this paper, the free water droplet motion on substrate actuated by surface acoustic waves (SAW) is modelled and experimentaly validated. This paper reports on measurements of the droplet velocity and its dependences from applied SAW power, frequencies. Experimentally observed water droplet of volume 2 and $5 \mu 1$ and their motion influenced by SAW with frequencies 34 and $58 \mathrm{MHz}$ for different power range 0-5 dbm. Results simulated using moving mesh finite element method (MM-FEM) of modelling. For the first time, a threedimensional (3D) time dependent finite element computational modeling of nonlaminar two-phase flow of the liquid droplet motion was developed and visualised using MM-FEM. The interaction of SAW with microliters of liquid is a fundamental theme for a small size fuel cells, and lab-on-chips application purposes.
\end{abstract}

Keywords: Water actuation, Surface Acoustic Waves, fluid flow, numerical simulation, modelling.

\section{Introduction}

Liquid motion induced from high-intensive acoustic waves is called acoustic streaming [1]. Surface acoustic waves generated on the surface of a piezoelectric device can actuate different liquids and this effect wide used on lab-on-a-chip devices. A novel type of microfluidic devices use surface acoustic waves (SAW) as a driving force. Surface acoustic waves are generated by the application of an alternating voltage signal to transducers patterned on a piezoelectric. Computational modelling such as finite-element method (FEM) present an alternative to the experiments and can be used for more numerical representation insides of the flow field, pressure, vorticity and velocity.

\section{Experiment}

The new SAW devices were made on the basis of a lithium niobate piezoelectric crystal $\left(\mathrm{LiNbO}_{3}\right)$ with a high electromechanical coupling coefficient the conversion coefficient of a high-frequency electrical signal into mechanical oscillations of the crystal lattice of a piezoelectric crystal in the form of SAW. To test basic characteristics of SAW devices for liquid displacement we fabricated SAW devices with various resonance frequencies. Fig. 1 shows the captured frames from video of the experimental results with the imposition of the scale bar to calculate the speed of the droplet. Comparison of the speed of movement of a water droplet with experiment shows that it gives a value of $2.25 \mathrm{~cm} / \mathrm{s}$, which is in good agreement with experimentally measured movement using a videotape (in the range of $1.43-2.85 \mathrm{~cm} / \mathrm{s}$ ).

In the experiment, the droplet speed was from $0.5 \mathrm{~cm} / \mathrm{s}$ to $4.5 \mathrm{~cm} / \mathrm{s}$, depending on the volume of droplet and the SAW power. The simulation results are important for clarifying the mechanism of interaction of SAW with a water droplet and for designing laboratory devices on chips. Based on the collected experimental data of droplet movement under action of SAW, the analysis of interdependencies between the supplied power (W), volumes of droplet $(\mu \mathrm{l})$, frequencies $(\mathrm{MHz})$, types of liquids (water, 50\% alcohol solution in water), the speed of movement of the droplet was carried out. We considered water droplet with a volume in the range of 2 and $5 \mu l$ and build graph of depndences (Fig. 2). 

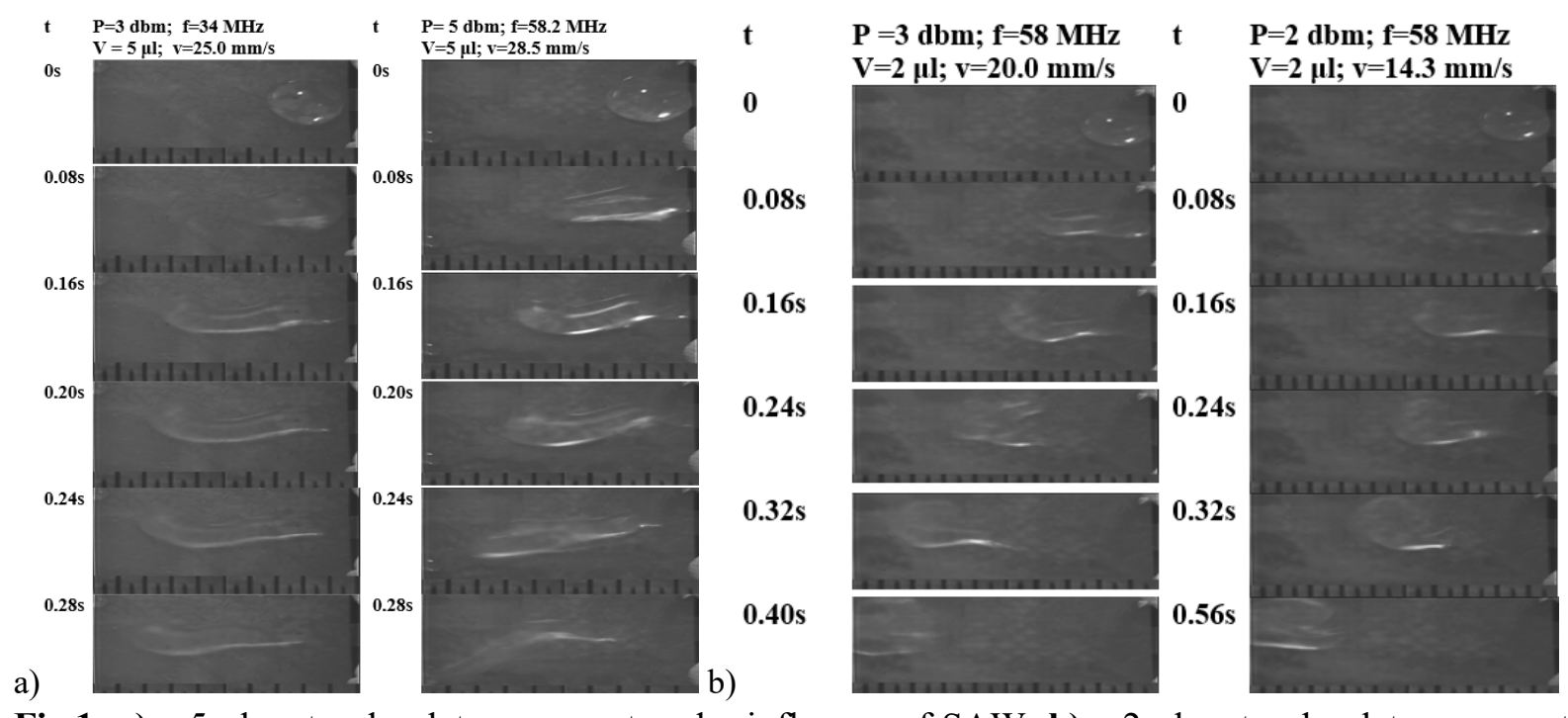

Fig.1: a) a $5 \mu$ water droplet movement under influence of SAW; b) a $2 \mu 1$ water droplet movement under influence of SAW.

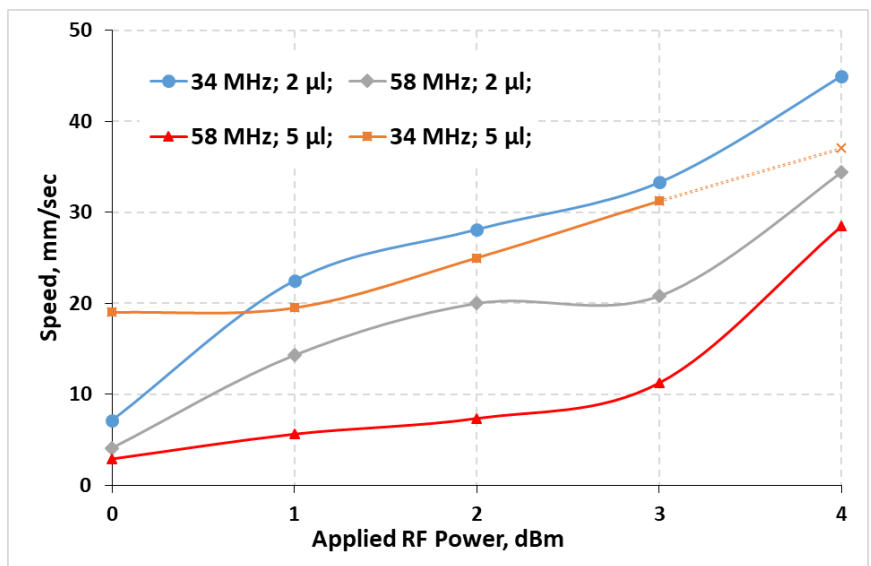

Fig. 2: - Applied frequency and water droplet speeds, $\mathrm{mm} / \mathrm{s}$

\section{FEM Modelling}

The numerical modeling of free water droplet moving under influence of SAW is carried out. To investigate this problem numerically, we have used the finite element method (FEM) using Comsol Multiphysics software. The program contains a library of partial differential equations and various tools for modeling and simulation. The COMSOL Multiphysics simulation platform helps to model the process by defining geometry, meshing sample, specifying physics, solving, and then visualizing results. These include tools for geometric constructions, mesh generators, different solvers that can help to quickly cope with even the most complex problems of linear and nonlinear problems, as well as post-processing. COMSOL Multiphysics allows solving multi-physical problems, which described by a combination of different partial differential equations and thereby the complex analysis of the physical model is carried out.

We have developed a three-dimensional mathematical model of propagation of surface acoustic waves on a Lithium Niobate piezoelectric substrate and water droplet movement under the influence of SAW. This model allows us to consider SAW motion processes on the surface of the substrate and calculate: fluid flow rate, flow velocity and voltage over time.

Modeling consists of two parts. For a start, a piezoelectric substrate with IDT was simulated to determine the eigenfrequencies localization of SAW at the solid-liquid interface. Then these frequencies were used to excite a variable RF signal at IDT with time-dependent analyze. It should be noted that the lower part was fixed and absorbed vibrations and did not reflect the waves. First, we simulated the propagation of surface acoustic waves 
on a Lithium Niobate piezoelectric substrate, and then developed a new computing model that excite the fluid motion using the first part of simulation surface acoustic waves (SAW) on the surface of the piezoelectric crystal.

For the first time, a three-dimensional (3D) mathematical model of the liquid droplet motion was developed. To describe the motion of the fluid flow, we used a Laminar Two-Phase Flow, Moving Mesh interface (TPFMM) in COMSOL Multiphysics 5.2 version with a time-dependent study which solves the Navier-Stokes equation (5) for incompressible fluid flow:

$$
\begin{gathered}
\rho \frac{\partial \boldsymbol{u}}{\partial t}+\rho(\boldsymbol{u} \cdot \nabla) \boldsymbol{u}=\nabla \cdot\left[-p \boldsymbol{I}+\mu\left(\boldsymbol{\nabla} \boldsymbol{u}+(\boldsymbol{\nabla u})^{T}\right)\right]+\boldsymbol{F} \\
\frac{\partial \rho}{\partial t}+\nabla \cdot(\rho \boldsymbol{u})=0
\end{gathered}
$$

where $\rho$ - the density (SI unit: $\mathrm{kg} / \mathrm{m}^{3}$ ); $\mathrm{u}$ - the velocity vector (SI unit: $\mathrm{m} / \mathrm{s}$ ); $\mathrm{p}$ - pressure (SI unit: Pa); F - the volume force vector (SI unit: N/m3); I - Identity matrix; $\mu$-dynamic viscosity (SI unit: $\mathrm{Pa}^{*}$ s).

Equation (5) is the momentum equation and (6) is the continuity equation. Here u denotes the mesh velocity and arises from the definition of time derivatives in the coordinate system of the deformed mesh. Poisson's equation is solved for mesh displacement.

Streaming effect was modeled for water droplet in the gas phase in normal conditions by using Finite Element Method (FEM) approach in three-dimension space and Moving Mesh interface were combined. The Navier Slip boundary condition used for the free surface of the fluid and contact touches, also gravity force taken into account. The mesh Model Builder with Swept Distribution calibrated to Fluid dynamics. The volume force vector components obtained according to Shiokawa, S., et. al. (1989) [2], Alghane, M., et. al. (2011) [3], Du X. Y., et.al. (2009)[4].

$$
\begin{aligned}
& F x=\rho\left(1+\alpha_{i}^{2}\right) A^{2} \omega^{2} k_{i} \cdot \exp \left(2\left(k_{i} x+\alpha_{i} k_{i} z\right)\right) \\
& F z=\rho\left(1+\alpha_{i}^{2}\right) A^{2} \omega^{2} \alpha_{i} k_{i} \cdot \exp \left(2\left(k_{i} x+\alpha_{i} k_{i} z\right)\right)
\end{aligned}
$$

where $\alpha_{i}=j \alpha, k_{L}=k_{r}+j k_{i} . F_{x}$ and $F_{z}$ are $x$ and $z$ component of volume force $\mathrm{F}$, respectively. Total SAW streaming force $F^{2}=F_{x}^{2}+F_{z}^{2}$. A is the SAW amplitude which described in [4].

On the solid-fluid interface, the boundary conditions for the emission of plane waves into the second medium are established. SAW is propagating across the substrate falls under the droplet. The model was shown that due to the difference in the elastic wave propagation velocities under Rayleigh angle of acoustic radiation of the energy is transferred by acoustic radiation into the liquid, so-called leaky waves. Further, the obtained solution is used in the next modeling stage - the equation of the convection wave, which is necessary for modeling the propagation of ultrasound by the CWE module in water. The process of excitation of ultrasound in a liquid depends on an external source; therefore, for CWE module, stitching conditions are set at the boundary. This analysis is needed in order to determine the local values of acceleration, velocity, and acoustic pressure in water. These data were useful for calculating the components of the total volume force (at the macroscale), which acts on the drop and falls in an exponential relationship as the acoustic radiation inside the drop. Further, using the laminar flow module in conjunction with a moving-mesh grid, the components of the volume force acting on the drop were applied. 


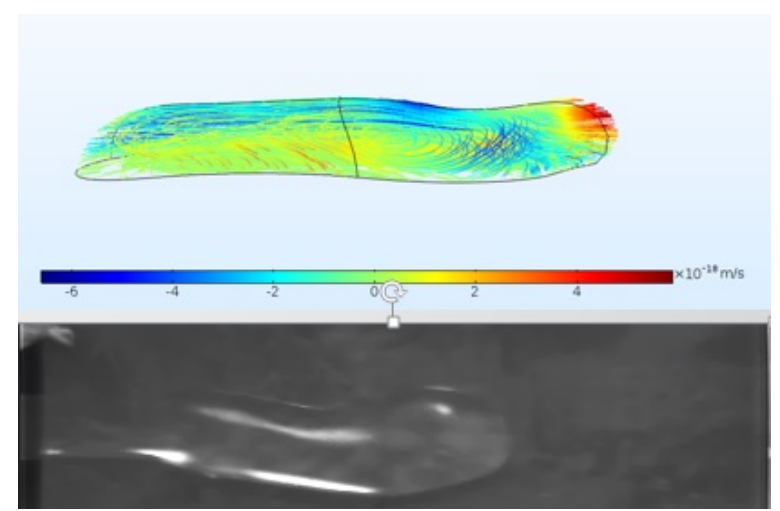

Fig. 3: The simulation results of water droplet motion under influence of SAW compared with experimental observation

a)

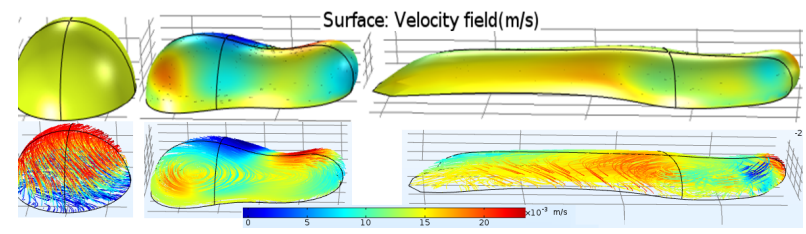

b)

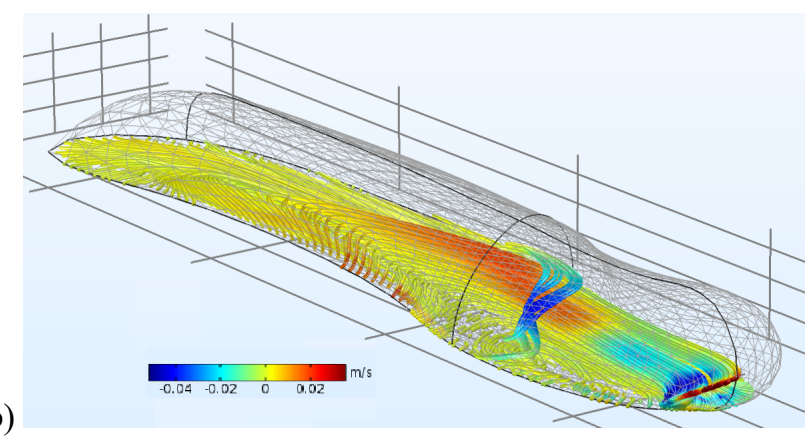

Fig. 4: a) 3D Numerical model of water droplet and internal streaming flows. b) Internal mixing visualization.

Corresponding parameters of dynamic viscosity, contact angle, and surface tension force were set. The simulation results show that the water droplet motion under the influence of SAW is consistent with the experimental observations in Fig. 3.

The simulation is achieved about 40 minutes. As a result, it is clear that, despite the physics of laminar flow, turbulence is used and internal streaming is observed (see Fig. 4). This is due to the fact that the volume force is non-uniform and varies in-depth and in height of the drop as it moves away from the source of the surface acoustic wave.

\section{Summary}

In summary, the numerical modelling of free water droplet motion under the influence of SAW is carried out. The water droplet motion induced by SAW was experimentally observed. Experimental results simulated using moving mesh finite element method (MM-FEM). For the first time, a three-dimensional (3D) time dependent finite element computational modeling of nonlaminar two-phase flow of the liquid droplet motion was developed and visualised using MM-FEM.

\section{Acknowledgements}

This work was supported by the World Science Stars program of Nazarbayev University. 
Proceedings of the Nanotech France 2019 International Conference

Paris, France - June 26 - 28, 2019

DOI: https://doi.org/10.26799/cp-nanotechfrance2019

\section{References}

1. W. Nyborg, Acoustic Streaming (Academic, New York, 1965)

2. S.Shiokawa, Y.Matsiu and T. Ueda, IEEE 0090-5607/89/0000-0643 Ultrasonics symposium 1989

3. M.Alghane, Y.Q.Fu, B.X. Chen, Y. Li, M.P.Y. Desmulliez, and A.J. Walton, J.Appl.Phys. 109,114901, 2011

4. Y. J. Guo, Y.Q. Fu et al., J. Appl. Phys. 116, 024501 (2014) 